Statistical Summary of Hydrologic and Water-Quality Data from the Halawa, Haiku, and Kaneohe Drainage Basins Before, During, and After H-3 Highway Construction, Oahu, Hawaii, 1983-99

U.S. GEOLOGICAL SURVEY

Open-File Report 01-64

Prepared in cooperation with the STATE OF HAWAII DEPARTMENT OF TRANSPORTATION and the FEDERAL HIGHWAY ADMINISTRATION 


\section{Statistical Summary of Hydrologic and Water-Quality Data from the Halawa, Haiku, and Kaneohe Drainage Basins Before, During, and After H-3 Highway Construction, Oahu, Hawaii, 1983-99}

By Michael F. Wong and Stacie T.M. Young

U.S. GEOLOGICAL SURVEY

Open-File Report 01-64

Prepared in cooperation with the

STATE OF HAWAII DEPARTMENT OF TRANSPORTATION

and the FEDERAL HIGHWAY ADMINISTRATION 


\section{U.S. DEPARTMENT OF THE INTERIOR}

Gale A. Norton, Secretary

\section{U.S. GEOLOGICAL SURVEY}

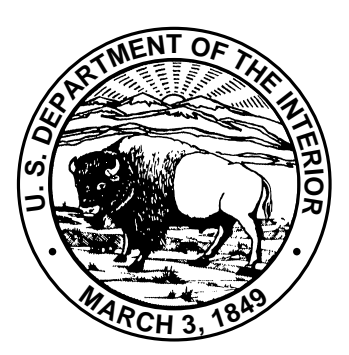

Charles G. Groat, Director

The use of firm, trade, and brand names in this report is for identification purposes only and does not constitute endorsement by the U.S. Geological Survey.

For additional information write to:

District Chief

U.S. Geological Survey

677 Ala Moana Blvd., Suite 415

Honolulu, HI 96813
Copies of this report can be purchased from:

U.S. Geological Survey

Branch of Information Services

Box 25286

Denver, CO 80225-0286 


\section{CONTENTS}

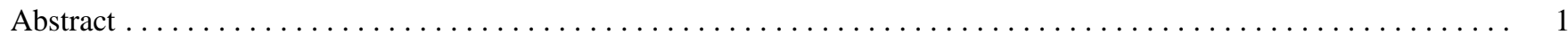

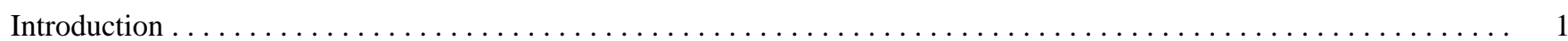

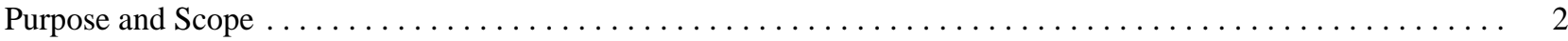

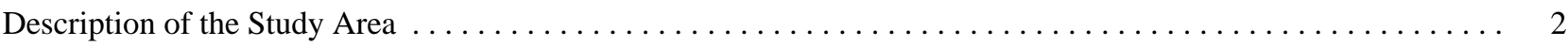

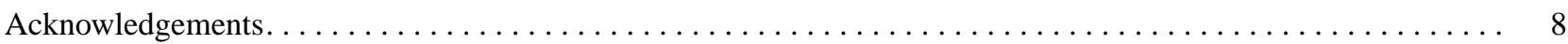

Methods of Hydrologic and Water-Quality Data Collection $\ldots \ldots \ldots \ldots \ldots \ldots$

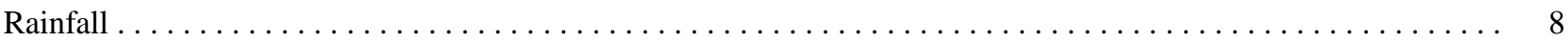

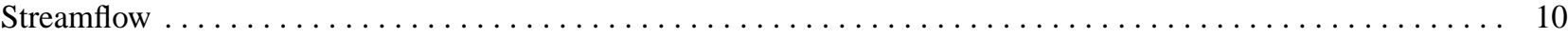

Suspended Sediment $\ldots \ldots \ldots \ldots \ldots \ldots \ldots \ldots \ldots \ldots \ldots \ldots \ldots \ldots \ldots \ldots \ldots$

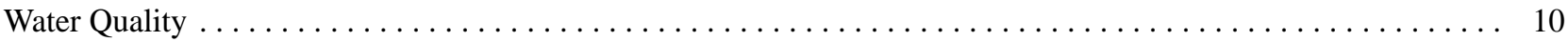

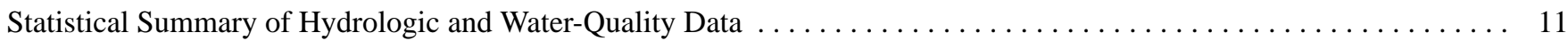

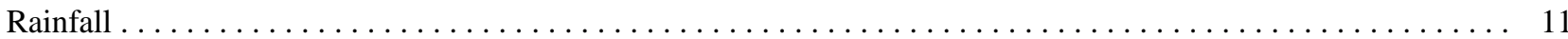

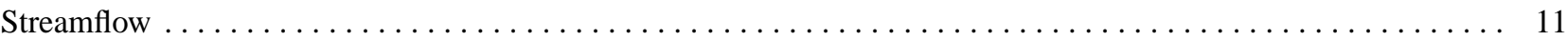

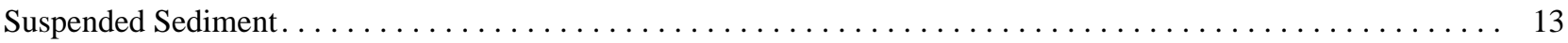

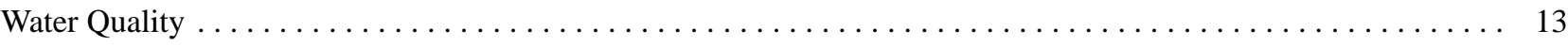

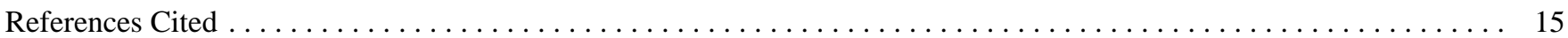

\section{FIGURES}

\section{1-2. Maps showing:}

1. Selected stream-gaging stations and drainage basins in the H-3 Highway study area, Oahu, Hawaii . . . 3

2. Haiku and Kaneohe drainage basins study area, Oahu, Hawaii. . . . . . . . . . . . . . . 4

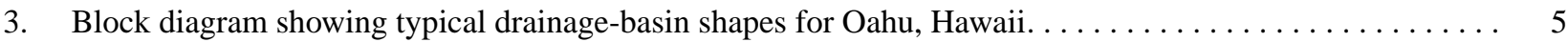

4. Flow-duration curves of daily flows for stations 16226200 North Halawa Stream and 16275000

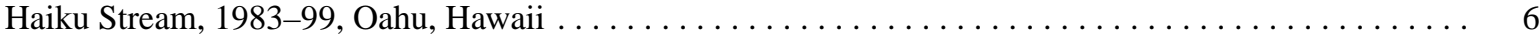

5. Diagram showing stream-gaging and water-quality stations in the Kaneohe drainage basin, Kaneohe,

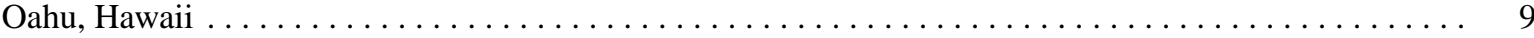

6. Total annual rainfall at Haiku rain gage 839.3, Oahu, Hawaii, water years $1987-98 \ldots \ldots$. . . . . . . . 12

7. Total annual streamflow at station 16226200, North Halawa Stream near Honolulu, Oahu, Hawaii,

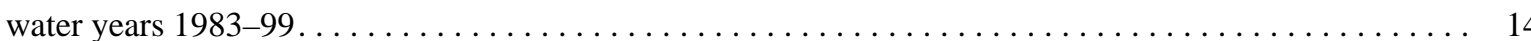




\section{TABLES}

1. Chronology of construction activities, H-3 Highway construction project, 1983-99, Oahu, Hawaii, . . . . . . . 17

2 Chronology of hydrologic and water-quality data-collection activities, H-3 Highway study area, Oahu, Hawaii. . . 18

3. Physiography of basin upstream from gaging stations in the H-3 Highway study area,

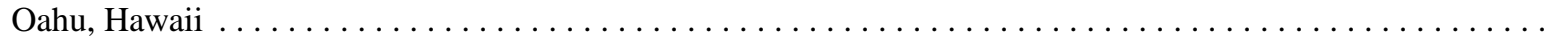

4. Average suspended-sediment particle-size analyses at stream-gaging stations in the H-3 Highway study area,

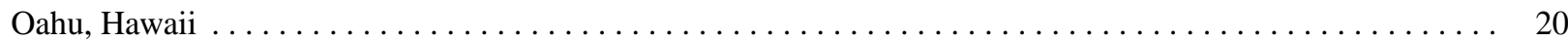

5. Total annual rainfall in the H-3 Highway study area, Oahu, Hawaii, water years $1983-99 \ldots \ldots \ldots$. . . . . . . . 20

6. Total annual streamflow at stream-gaging stations in the H-3 Highway study area, Oahu, Hawaii,

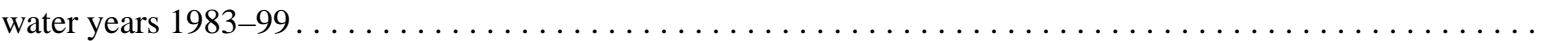

7. Total annual streamflow per unit area at stream-gaging stations in the H-3 Highway study area, Oahu, Hawaii,

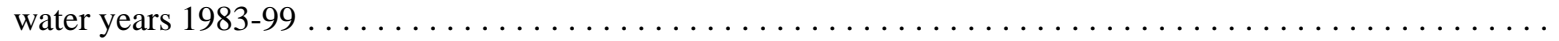

8. Summary of suspended-sediment concentrations at stream-gaging stations in the H-3 Highway study area,

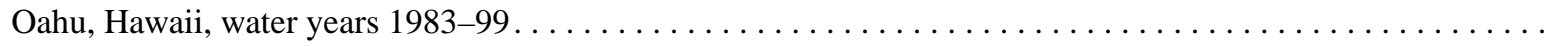

9. Total annual suspended-sediment loads at stream-gaging stations in the H-3 Highway study area, Oahu, Hawaii,

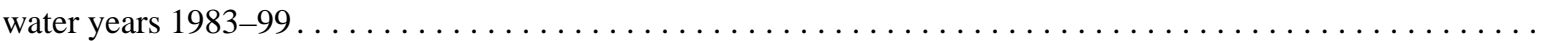

10. Total annual suspended-sediment loads per unit value of total streamflow at stream-gaging stations in the

H-3 Highway study area, Oahu, Hawaii, water years 1983-99.

11. Total annual suspended-sediment yields at stream-gaging stations in the H-3 Highway study area, Oahu,

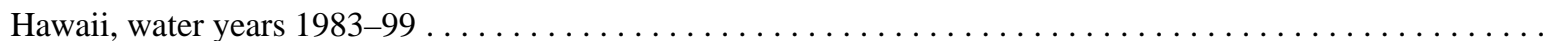

12-30. Statistical summary of selected water-quality data collected at station:

12. 16226200, North Halawa Stream near Honolulu, Oahu, Hawaii, from May 1983 to March 1999. . . . . .

13. 16227100, Halawa Stream below H-1, Oahu, Hawaii, from November 1988 to November 1998

14. 16265600, Right Branch Kamooalii Stream near Kaneohe, Oahu, Hawaii, from February 1983 to January 1998

15. 16265700 , Kamooalii Stream at alt $200 \mathrm{ft}$ near Kaneohe, Oahu, Hawaii, from February 1983 to

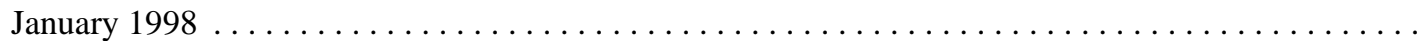

16. 16266500, Hooleinaiwa Stream at alt $220 \mathrm{ft}$ near Kaneohe, Oahu, Hawaii, from February 1983 to

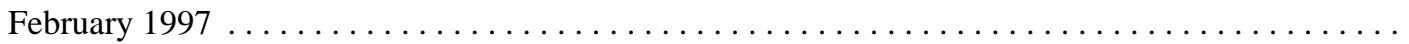

17. 16267500, Hooleinaiwa Stream above confluence with Kamooalii Stream near Kaneohe, Oahu, Hawaii, from February 1983 to January $1998 \ldots \ldots \ldots \ldots \ldots \ldots \ldots \ldots \ldots \ldots \ldots \ldots \ldots$

18. 16269500, Kuou Stream at alt $220 \mathrm{ft}$ near Kaneohe, Oahu, Hawaii, from February 1983 to January 1998

19. 16270900, Luluku Stream at alt $220 \mathrm{ft}$ near Kaneohe, Oahu, Hawaii, from February 1983 to April 1998

20. 16272200, Kamooalii Stream below Luluku Stream near Kaneohe, Oahu, Hawaii, from February 1983

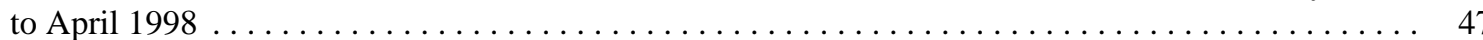

21. 16273950, South Fork Kapunahala Stream at Kaneohe, Oahu, Hawaii, from May 1988 to April 1998 . . 50 
22. 16274100, Kaneohe Stream below Kamehameha Highway at Kaneohe, Oahu, Hawaii, from

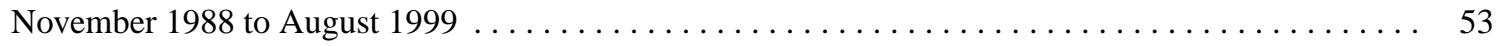

23. 16275000, Haiku Stream near Heeia, Oahu, Hawaii, from March 1983 to April 1998 . . . . . . . . . 56

24. 212335157482603, Waimaluhia Reservoir cross-section 1-3 (composite) near Kaneohe, Oahu,

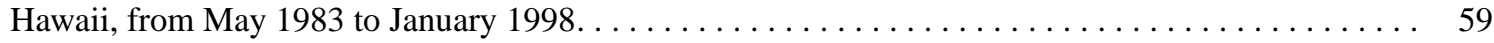

25. 212335157482603, Waimaluhia Reservoir cross-section 1-3 at $1 \mathrm{ft}$ depth near Kaneohe, Oahu,

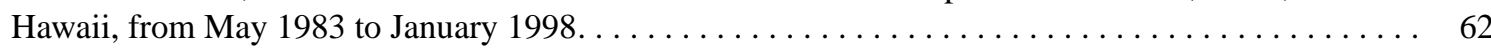

26. 212335157482603, Waimaluhia Reservoir cross-section 1-3 at $7 \mathrm{ft}$ depth near Kaneohe, Oahu,

Hawaii, from May 1983 to January $1998 \ldots \ldots \ldots \ldots \ldots \ldots \ldots \ldots \ldots \ldots \ldots$

27. 212335157482603, Waimaluhia Reservoir cross-section 1-3 at $11 \mathrm{ft}$ depth near Kaneohe, Oahu,

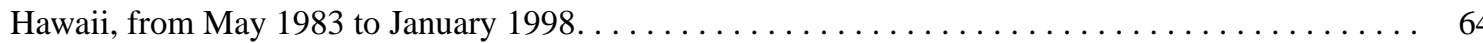

28. 212329157483102, Waimaluhia Reservoir cross-section 3-2 at $1 \mathrm{ft}$ depth near Kaneohe, Oahu,

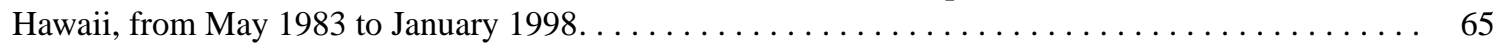

29. 212329157483102, Waimaluhia Reservoir cross-section 3-2 at $5 \mathrm{ft}$ depth near Kaneohe, Oahu,

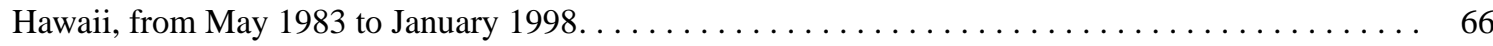

30. 212329157483102, Waimaluhia Reservoir cross-section 3-2 at $10 \mathrm{ft}$ depth near Kaneohe, Oahu,

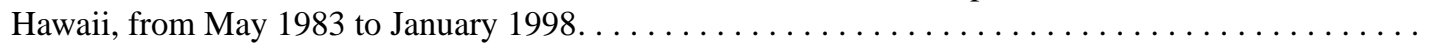

31. Pesticides, herbicides, and polychlorinated compounds analyzed for at stream-gaging and water-quality

stations in the H-3 Highway study area, Oahu, Hawaii, from February 1983 to August 1999 . . . . . . . . . . .

32. Pesticides, herbicides, and polychlorinated compounds detected at stream-gaging and water-quality

stations in the H-3 Highway study area, Oahu, Hawaii, from February 1983 to August 1999

\begin{tabular}{|c|c|c|}
\hline Multiply & By & To obtain \\
\hline \multicolumn{3}{|c|}{ Length } \\
\hline inch (in) & 2.54 & centimeter \\
\hline foot $(\mathrm{ft})$ & 0.3048 & meter \\
\hline \multicolumn{3}{|c|}{ Area } \\
\hline acres & 4,047 & square meter \\
\hline square mile $\left(\mathrm{mi}^{2}\right)$ & 2.590 & square kilometer \\
\hline square mile $\left(\mathrm{mi}^{2}\right)$ & 640 & acres \\
\hline \multicolumn{3}{|c|}{$\begin{array}{l}\text { Volume per unit time } \\
\text { (includes flow) }\end{array}$} \\
\hline cubic foot per second $\left(\mathrm{ft}^{3} / \mathrm{s}\right)$ & 0.02832 & cubic meter per second \\
\hline cubic foot per second-days ( $\mathrm{ft}^{3} / \mathrm{s}$-days) & 0.02832 & cubic meter per second-days \\
\hline \multicolumn{3}{|c|}{ Weight } \\
\hline ton (ton) & 0.9072 & metric ton \\
\hline ton per square mile (ton $/ \mathrm{mi}^{2}$ ) & 0.3503 & metric ton per square kilometer \\
\hline
\end{tabular}


Temperature in degrees Fahrenheit $\left({ }^{\circ} \mathrm{F}\right)$ may be converted to degrees Celsius $\left({ }^{\circ} \mathrm{C}\right)$ as follows:

$$
{ }^{\circ} \mathrm{C}=\left({ }^{\circ} \mathrm{F}-32\right) / 1.8
$$

Temperature in degrees Celsius $\left({ }^{\circ} \mathrm{C}\right)$ may be converted to degrees Fahrenheit $\left({ }^{\circ} \mathrm{F}\right)$ as follows:

$$
{ }^{\circ} \mathrm{F}=1.8\left({ }^{\circ} \mathrm{C}\right)+32
$$

Abbreviations used: $\mu \mathrm{S} / \mathrm{cm} @ 25^{\circ} \mathrm{C}$, microsiemens per centimeter at $25^{\circ} \mathrm{C}$

NTU, nephelometric turbidity units

$0.7 \mu \mathrm{m}-\mathrm{MF}, 0.7$-micron membrane-filter method

$\mathrm{mg} / \mathrm{L}$, milligrams per liter

$\mu \mathrm{g} / \mathrm{L}$, micrograms per liter 


\title{
Statistical Summary of Hydrologic and Water-Quality Data from the Halawa, Haiku, and Kaneohe Drainage Basins Before, During, and After H-3 Highway Construction, Oahu, Hawaii, 1983-99
}

\author{
By Michael F. Wong and Stacie T.M. Young
}

\begin{abstract}
This report provides statistical summaries of rainfall, streamflow, suspended-sediment, and water-quality data collected in the Halawa, Haiku, and Kaneohe drainage basins before, during, and after construction of the H-3 Highway on the island of Oahu, Hawaii. Methods of data collection also are described. Data collected during water years 1983 through 1999 at eight streamflow and six stream water-quality gaging-stations, and two water-quality stations located in Waimaluhia Reservoir are included. Physiographic data for all basins contributing to the 14 stream stations as well as brief land-use descriptions of the Halawa, $\mathrm{Haiku}$, and Kaneohe drainage basins are provided.
\end{abstract}

\section{INTRODUCTION}

The construction of the H-3 Highway across the Koolau Range on the island of Oahu, Hawaii (fig. 1) raised concerns over the potential effects of the construction on streamflow, sediment transport, and quality of surface water in the drainage basins affected by the construction. In 1983, the U.S. Geological Survey, in cooperation with the State of Hawaii Department of Transportation and the Federal Highway Administration, began a study of the streamflow, sediment, and water-quality characteristics of these surface waters. The purpose of this study was to determine if construction activities were affecting streams along the highway right-of-way, and if so, the magnitude and extent of construction effects. Data collection was carried out before, during, and after construction of the H-3 Highway.

The H-3 Highway route traverses the drainage basins of Halawa and Haiku Streams and the tributaries of Kaneohe Stream (fig. 1). The H-3 extends from the H-1 Highway near the East Loch of Pearl Harbor on the leeward (southwestern) side of the Koolau Range to the Halekou Interchange on the windward (northeastern) side, where the H-3 connects to a previously constructed section of H-3 leading from Kamehameha Highway to Kaneohe Marine Corps Base-Hawaii. The completed H-3 Highway consists of part cut-and-fill and part viaduct sections in the lower North Halawa Valley, a viaduct through the upper North Halawa Valley, twin tunnels below the crest of the Koolau Range, a viaduct through the Haiku drainage basin, and a cutand-fill section through the Kapunahala and Kamooalii drainage sub-basins of the Kaneohe drainage basin (U.S. Department of Transportation, Federal Highway Administration and State of Hawaii Department of Transportation, 1987). Highway construction affected from 3 to 11 percent of the drainage basin areas upstream of the stream-gaging stations discussed in this report.

Construction of the H-3 Highway proceeded in increments (table 1-all tables are at the end of the report). Construction was at times halted by court actions, and the planned route was modified to avoid sites of cultural importance. Access roads were built in the North Halawa and Haiku Valleys before construction of the highway. An exploratory tunnel was 
excavated below the crest of the Koolau Range before the larger, traffic tunnels were excavated.

Erosion-control measures were used throughout construction of the H-3 Highway to reduce sediment delivery from construction areas in all affected drainage basins. These measures included erosion-cloth barriers installed on hillslopes and along channels, loose-rock check dams in channels, and hydromulching and installation of plastic netting on cut-and-fill slopes. Streamflow in several channel reaches in upper North Halawa Valley was diverted into culverts, which were then buried, during construction to prevent sediment disturbed by construction from reaching the stream. Sections of North Halawa Stream in the upper valley also were channelized as part of the highway construction.

\section{Purpose and Scope}

This report describes the Halawa, Haiku, and Kaneohe drainage basins, the methods used for hydrologic and water-quality data collection, and statistical summaries of the hydrologic and water-quality data collected during the 17-year (1983-99) study period. Data are presented for the Halawa (station 227100), North Halawa (stations 225800, 226000, and 226200), Haiku (station 275000), South Fork Kapunahala (station 273950), Kamooalii (stations 265600, 265700, 266500, 267500, 269500, 270900, and 272200), and Kaneohe (station 274100) drainage basins (figs. 1 and 2). Sixdigit station numbers used in this report are abbreviated numbers. Complete station numbers are preceded by 16 . For example, for station 225800 the complete number is 16225800. Water-quality data also are presented for two locations in the Waimaluhia Reservoir. Different types of data were collected at various times at the above stations. Table 2 summarizes the dates that streamflow, suspended-sediment, and water-quality data were collected at the stations. This report updates a previously published statistical summary of data collected during water years 1983-89 (Wong and Hill, 1992).

Daily values of streamflow, suspended-sediment concentration, particle-size, and load, and instantaneous water-quality data, that are statistically summarized in this report, have been published in the U.S. Geological Survey's annual water-resources data reports for Hawaii, water years 1983 through 1999. For example see Hill and others (2000) for the water year 1999 report. A water year is a 12-month period that extends from October 1 through September 30 and is designated by the calender year in which it ends.

\section{Description of the Study Area}

The study area for this report consists of the Halawa drainage basin on the leeward or western side of the Koolau Range (fig. 1) and the Haiku and Kaneohe drainage basins (fig. 2) on the windward or eastern side. In the Halawa drainage basin, most of the data were collected in the North Halawa drainage basin (fig. 1), while in the Kaneohe drainage basin, most of the data were collected in the South Fork Kapunahala and Kamooalii drainage basins (fig. 2). The Kamooalii drainage basin was further divided with extensive data collection in the Luluku and Right Branch Kamooalii drainage basins (fig. 2).

Physiography and lithology.--The Koolau Range is the eroded remnant of the larger and younger of the two major shield volcanoes that formed the island of Oahu (Visher and Mink, 1964). Much of the windward side of the original Koolau Volcano has been eroded, leaving a steep windward slope indented with short, broad, amphitheater-shaped valleys (Hinds, 1925). This is in sharp contrast to the gentle leeward slopes which are deeply dissected by long roughly linear valleys (fig. 3 ). Ridges on the leeward side approximate the original morphology of the Koolau volcanic dome (Wentworth, 1943). Selected physiographic data for the drainage basins upstream of the 14 gaging stations and Waimaluhia Reservoir are listed in table 3. Physiographic measurements were made manually using the U.S. Geological Survey's 1983, 1:24,000 scale, 7.5 minute topographic quadrangle maps of Oahu. Values in table 3 show that the study drainage basins are all small in size and have steep channel gradients.

Lithology of the study area consists primarily of basalt that was extruded in numerous, gently dipping, thin (less than $10 \mathrm{ft}$ ) flows of aa and pahoehoe lava (Visher and Mink, 1964). These flows are intruded by near-vertical dikes in the rift zone near the crest of the present Koolau Range (Visher and Mink, 1964). More recent volcanic rocks are exposed in small areas on the windward side of the study area (Takasaki and others, 1969). The gently sloping lower parts of the windward drainage basins and the valley floor of the North Halawa drainage basin are overlain by alluvium derived 


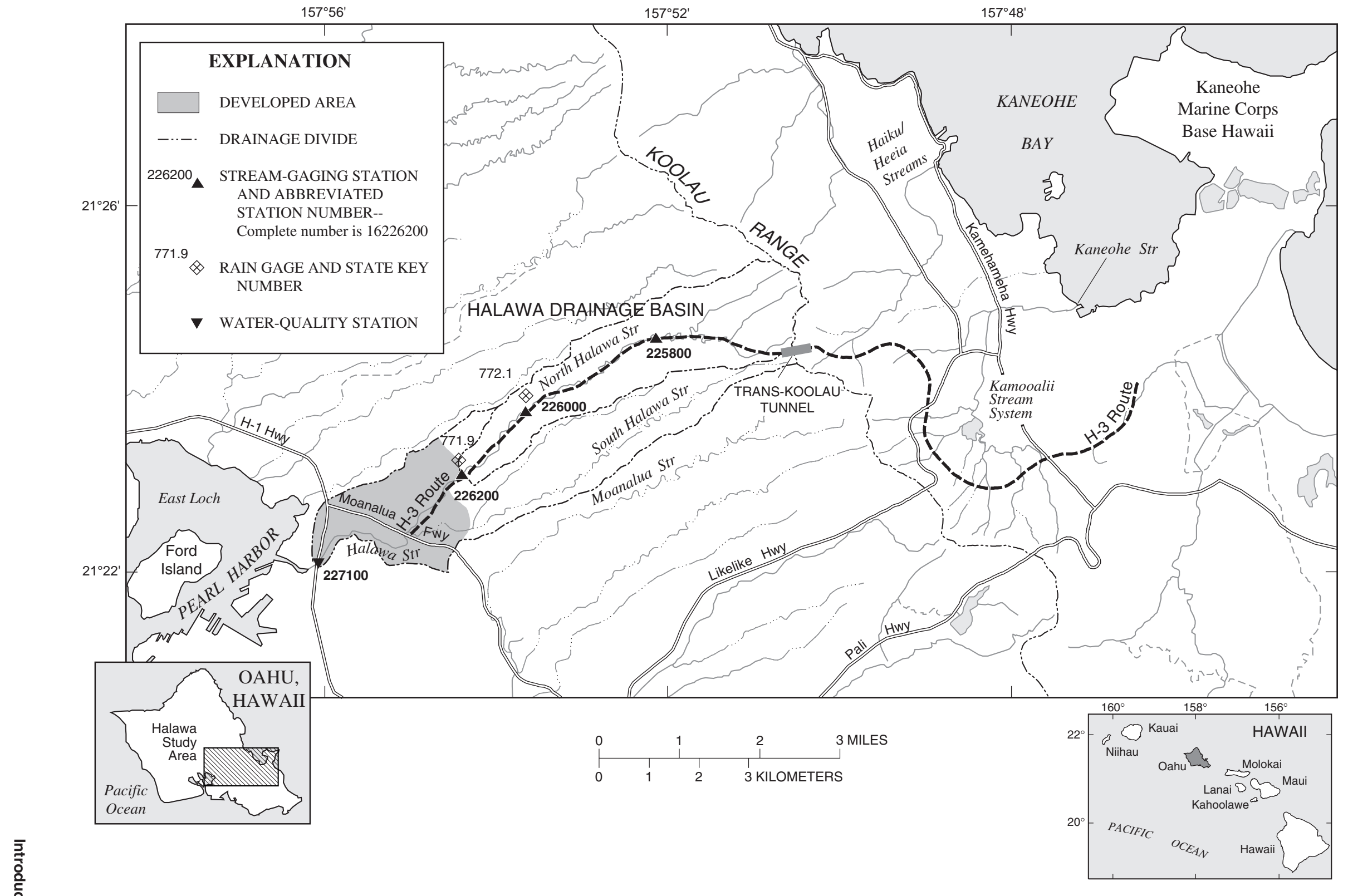

Figure 1. Selected stream-gaging stations and drainage basins in the H-3 Highway study area, Oahu, Hawaii. 


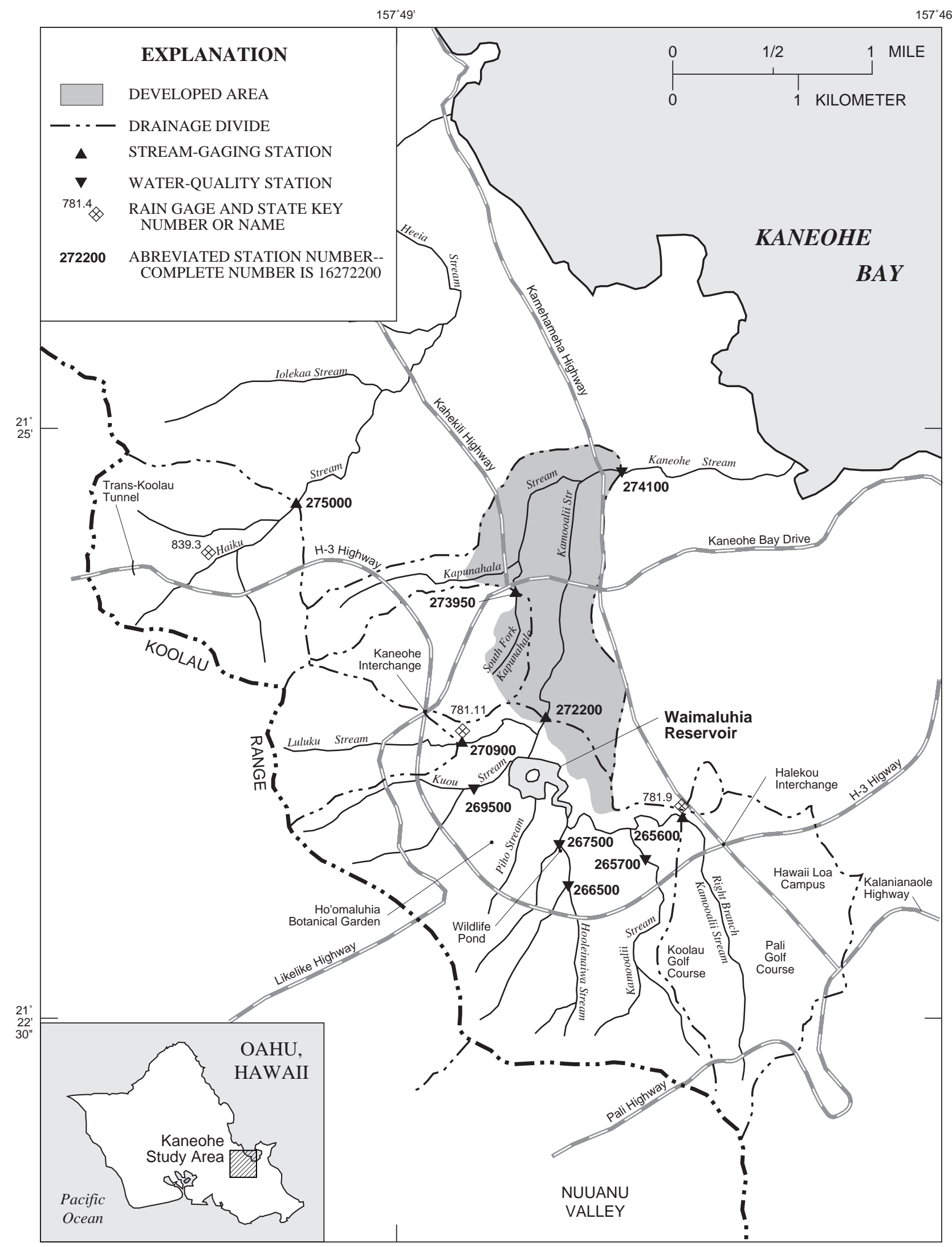

Figure 2. Haiku and Kaneohe drainage basins study area, Oahu, Hawaii. 


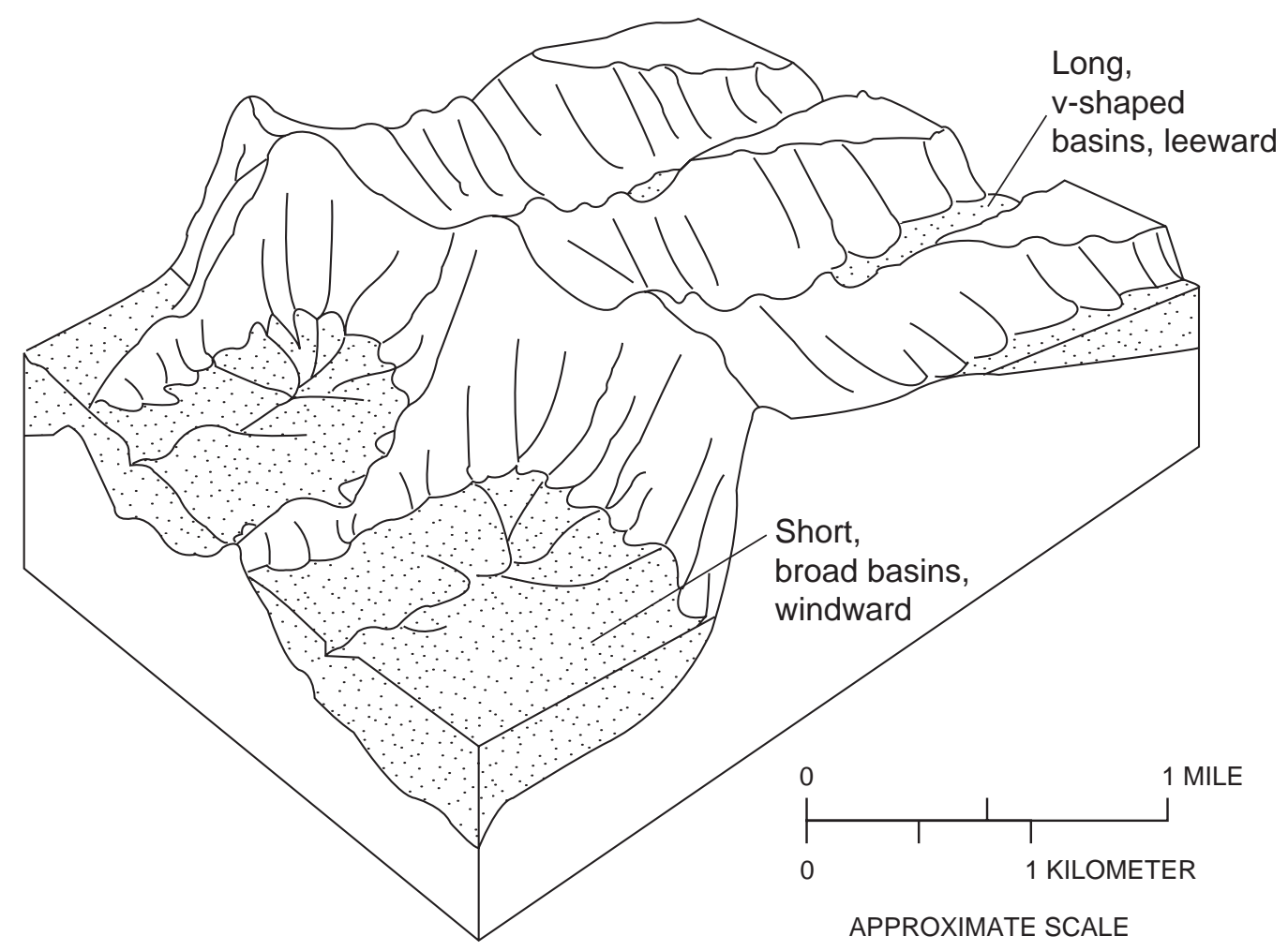

Figure 3. Block diagram showing typical drainage-basin shapes for Oahu, Hawaii (by S.K. Izuka, U.S. Geological Survey).

from erosion of the Koolau Range (Takasaki and others, 1969; Izuka, 1992).

Climate and streamflow.--The climate of Oahu is warm and humid. Average annual temperature ranges from $74^{\circ}$ to $76^{\circ} \mathrm{F}$ in the study area; temperatures above $95^{\circ} \mathrm{F}$ and below $50^{\circ} \mathrm{F}$ are rare (Blumenstock and Price, 1961). The distribution of rainfall is affected by the prevailing northeasterly trade winds and the topography of the island. Orographic lifting and cooling of marine air masses moving with the trade winds result in heavier and more frequent rainfall on the windward side and near the crest of the Koolau Range. The heaviest rainfall occurs about 0.5 to $1 \mathrm{mi}$ leeward of the crest (C.K. Wentworth, written commun., 1942; Mink, 1960). Rainfall varies seasonally, with most rainfall falling between November and April. Mean annual rainfall ranges from 75 to $150 \mathrm{in}$. on the windward side of the study area, and from 40 to more than $150 \mathrm{in.}$ on the leeward side (Blumenstock and Price, 1961). Mean annual pan evaporation is between 50 to $70 \mathrm{in}$. on the windward side and between 30 to $80 \mathrm{in}$. on the leeward side of the study area (Ekern and Chang, 1985).
The temporal and spatial distribution of streamflow is determined to a large extent by geology and climate. Streams respond rapidly to direct runoff that takes place during heavy rainfall. Large quantities of rainfall and the presence of numerous low-permeability dikes result in storage of large quantities of high-level ground water. These high-level reservoirs maintain the base flow of streams on the windward side (Takasaki and others, 1969). Streamflow in Halawa Stream is intermittent and is dependent primarily on direct runoff that occurs during heavy rainfall. Most infiltrating rainfall in the North Halawa Valley percolates to the basal aquifer and does not maintain base flows in the streams located there (Izuka, 1992). Streamflow is supplemented by the discharge of small quantities of ground water from alluvial aquifers that extend recession flows only to a minor extent (Izuka, 1992). The contrast between streamflow characteristics in the leeward and windward drainage basins of the study area is shown by using flow-duration curves (fig. 4). A flow-duration curve indicates the percentage of time that values of daily mean streamflow were equalled or exceeded (Searcy, 1959). The steep 


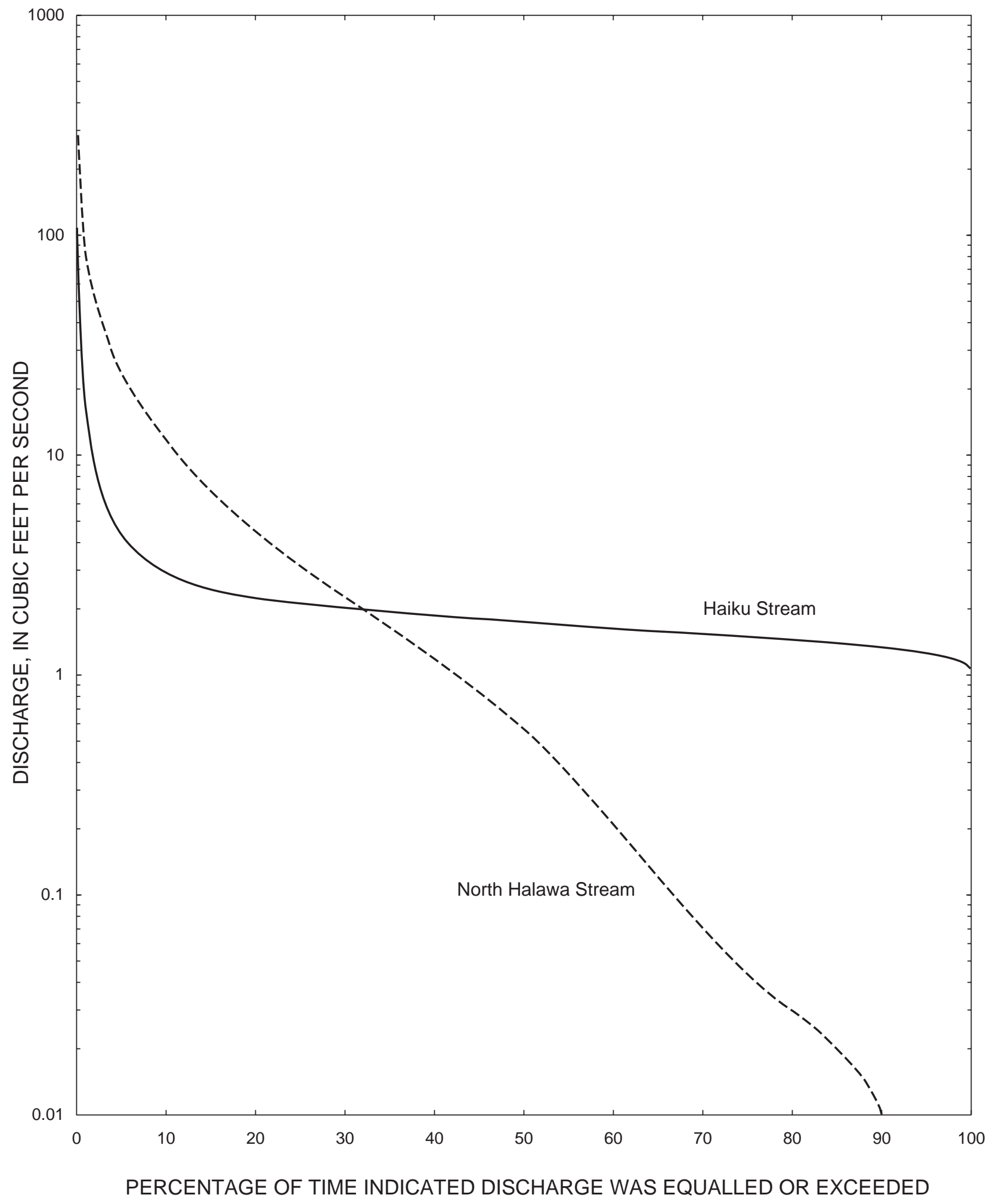

Figure 4. Flow-duration curves of daily flows for stations 16226200 North Halawa Stream and 16275000 Haiku Stream, 1983-99, Oahu, Hawaii. 
slope of the North Halawa Stream flow-duration curve indicates the highly variable nature of streamflow in Halawa Valley, whereas the flat slope of the Haiku Stream curve indicates the large storage of ground water on the windward side (fig. 4).

Soils and vegetation.--Soils in the Halawa drainage basin are classified as low permeability, very stony clays of the Kaena series with Kawaihapai clay loam soils by the mouth of Halawa Stream (Foote and others, 1972). Soils in the Haiku and Kaneohe drainage basins are classified as Lokelaa with some Kaneohe and Hanalei series silty clays. These types of silty clays have moderate permeability and are highly erodible on steep slopes (Foote and others, 1972). The majority of the mountainous areas within all the study drainage basins are classified as "rough mountainous land," "rock land," or "rock outcrop" (Foote and others, 1972), and little information is available on the soils of these areas.

Except where disturbed by development (figs. 1 and 2), all study basins are completely covered with one form or another of vegetation. Most of the native vegetation on the windward side of the study area has been replaced by cultivated crops, other non-native plants, and residential and commercial developments. Much of the Luluku sub-drainage basin (station 270900, fig. 2) has been converted to banana plantations. Vegetation in the North Halawa Valley is representative of undisturbed forest in the leeward Koolau Range and includes native and introduced species.

Halawa drainage basin.--The Halawa drainage basin is on the leeward side of the crest of the Koolau Range and has a drainage area of $8.90 \mathrm{mi}^{2}$ upstream of station 227100. Halawa Stream flows into the East Loch of Pearl Harbor and originates at the confluence of North and South Halawa Streams, downstream of Moanalua Freeway (fig. 2). Data collection in the Halawa study basin was concentrated in the North Halawa Valley. The water-quality data collected at station 227100, on Halawa Stream (fig. 1), was the only data collected outside of North Halawa Valley during the study. Between stations 227100 and 226200 the land is developed and consists of a light industrial park near the mouths of both North and South Halawa Streams and residential areas near the Moanalua Freeway and H-1 Highway. The H-3 Highway constitutes about 4 percent of the North Halawa $4.01 \mathrm{mi}^{2}$ drainage area upstream of station 226200. Before highway construction in the val- ley began in November 1987, this drainage basin was undeveloped.

Haiku drainage basin.--The Haiku drainage basin is on the windward side of the Koolau Range and adjoins the North Halawa drainage basin along the crest of the Koolau Range (fig. 1). At stream-gaging station 275000, Haiku Stream flows perennially. Haiku Stream joins Iolekaa Stream downstream of station 275000 to form Heeia Stream, which flows into Kaneohe Bay (fig. 2). Almost all of the $0.97 \mathrm{mi}^{2}$ drainage basin upstream of station 275000 was part of a U.S. Coast Guard navigational facility which was operated from 1944 to September 30, 1997. Highway construction in the Haiku drainage basin began in October of 1988 (table 1). The part of the H-3 Highway located within the Haiku drainage basin was constructed entirely as a viaduct, and covers about 3 percent of the drainage area upstream from station 275000.

Kaneohe drainage basin.--The Kaneohe drainage basin is on the windward side of the Koolau Range, south of the Haiku drainage basin, and has an area of $5.22 \mathrm{mi}^{2}$ upstream of station 274100. Kaneohe Stream flows into Kaneohe Bay downstream from the confluence of Kapunahala and Kamooalii Streams which join to form Kaneohe Stream near Kamehameha Highway (fig. 2). Data collected in the study basin was concentrated in the Kapunahala and Kamooalii sub-drainage basins. Only water-quality data was collected at station 274100 on Kaneohe Stream (fig. 2). Land use upstream of station 274100 and downstream of stations 272200 and 273950 consists entirely of residential and urban areas of Kaneohe town.

In the Kapunahala sub-drainage basin, only the South Fork of Kapunahala Stream was monitored during the study. The South Fork Kapunahala sub-drainage basin lies to the southeast of the Haiku drainage basin and has a drainage area of $0.40 \mathrm{mi}^{2}$ upstream from station 273950 , located at an altitude of $111 \mathrm{ft}$ (fig. 2). The drainage basin consists of residential and some agricultural lands in the lower portions of the basin. H-3 Highway construction in the basin began in March 1989 (table 1) with most of the construction activities occurring near the $400 \mathrm{ft}$ altitude. The H-3 Highway covers about 3 percent of the drainage area upstream from station 273950.

The Kamooalii sub-drainage basin upstream of stream-gaging station 272200 , at an altitude of $116 \mathrm{ft}$, has an area of $3.81 \mathrm{mi}^{2}$ (fig. 2). Land use upstream of 
the station includes the Hoomaluhia Botanical Garden, two golf courses, banana plantations, the Hawaii Loa Campus of Hawaii Pacific University, a small area of residential development, and undeveloped land. Parts of both Likelike and Pali Highways cross the drainage basin near the Koolau Range (fig. 2).

Highway construction within the Kamooalii subdrainage basin began in 1983 (table 1) with the construction of the Halekou Interchange upstream of station 265600 on the right branch of Kamooalii Stream (fig. 2). Numerous court injunctions delayed the construction at various times (table 1). Construction of the Windward Highway segment of the H-3 Highway, which affects most of the basin, began in the summer of 1989 and ended in the summer of 1992 (table 1). Most of the highway segments in the Kamooalii sub-drainage basin were constructed using cut and fill techniques. The H-3 Highway constitutes about 4 percent of the drainage area upstream from station 272200 .

In addition to station 272200 , streamflow, suspended-sediment, and water-quality data were collected in the Kamooalii sub-drainage basin at station 265600 on the Right Branch of Kamooalii Stream and at station 270900 on Luluku Stream. Streamflow at stations 272200, 265600, and 270900 is perennial. Streamflow at station 272200 includes water flowing through Waimaluhia Reservoir, a flood-control reservoir completed in 1981 upstream of the confluence of Luluku and Kamooalii Streams, and water from Luluku Stream that does not pass through the reservoir. The area affected by the H-3 Highway construction includes about 11 percent of the drainage area for station 270900 and 5 percent of the drainage area for station 265600. Additional water-quality data were collected at 12 sites in Waimaluhia Reservoir (fig. 5) and at four waterquality partial-record stations upstream of the reservoir: station 265700, Kamooalii Stream at altitude $200 \mathrm{ft}$, station 266500, Hooleinaiwa Stream at altitude $220 \mathrm{ft}$, station 267500, Hooleinaiwa Stream above confluence with Kamooalii Stream, and station 269500, Kuou Stream at altitude $220 \mathrm{ft}$ (figs. 2 and 5).

\section{Acknowledgements}

The authors gratefully acknowledge retired U.S. Geological Survey employees Harold Sexton, George Dayag and Frank Romualdo for their assistance with instrumentation and data collection. Mr. Ted Kamisato of Kaneohe, assisted in collecting suspended-sediment samples at station 265600. The staff at the Hoomaluhia Botanical Garden, Department of Parks and Recreation, City and County of Honolulu provided access to Waimaluhia Reservoir and provided boats used for field data collection. Start and end dates for construction activities were provided by Dennis Higa, Earl Kobataki, and Emilio Barroga Jr. of the State of Hawaii Department of Transportation. Stream-gaging stations 226000 , 275000 , and 272200 were operated in cooperation with the State of Hawaii Commission on Water Resource Management, Honolulu Board of Water Supply, and the U.S. Army Corps of Engineers, respectively. Suspended-sediment data collection from 1976 to 1997 at station 272200 was collected in cooperation with the U.S. Army Corps of Engineers.

\section{METHODS OF HYDROLOGIC AND WATER- QUALITY DATA COLLECTION}

Rainfall, streamflow, suspended-sediment, and water-quality data were collected at the network of stream-gaging and water-quality stations described above (figs. 1, 2, and 5). Stream-gaging stations were equipped with stage recorders and automatic suspended-sediment samplers except at station 226000 , where sediment data were not collected. In addition, stations 226000, 226200, 265600, and 270900 were equipped with recording rain gages. An additional rain gage was operated in Haiku Valley (fig. 2).

\section{Rainfall}

Rainfall was recorded using two types of rain gages. Both types used the standard National Weather Service 8-inch rain collector. At stream-gaging stations 226200, 265600, and 270900, tipping-bucket rain gages were used for most data collection. A tipping-bucket gage records the times at which set increments of rainfall have accumulated. All tipping-bucket gages in this study used 0.1 in. calibrated buckets and cumulative storage cans. The cumulative storage cans capture rainfall released when the bucket is tipped and provides a volumetric check on accumulated rainfall totals. At stream-gaging station 226000 and at the Haiku rain gage, a float-type rain gage was used. The float-type rain gage was used beginning in March 1997, January 1997, and July 1998 at stations 226200, 265600, and 


\section{EXPLANATION}

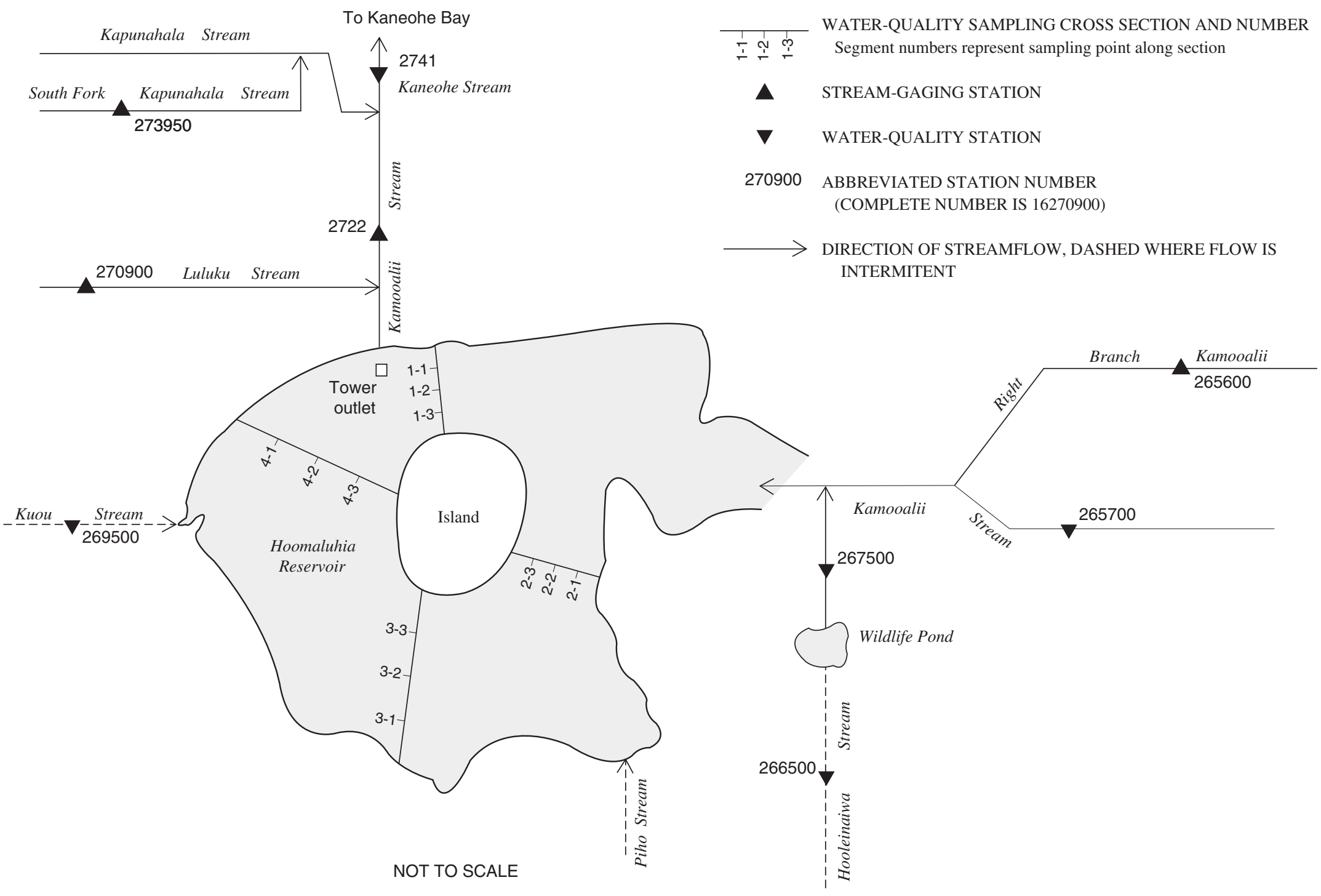

Figure 5. Diagram showing stream-gaging and water-quality stations in the Kaneohe drainage basin, Kaneohe, Oahu, Hawaii. 
270900, respectively, in place of the tipping-bucket gages. A float-type gage records continuous changes in rainfall volumes that are captured in a storage can connected to the 8 -in. rain collector.

\section{Streamflow}

Direct measurements of streamflow were made periodically at all stream-gaging and stream waterquality stations using standard practices for currentmeter measurements (Rantz and others, 1982). Peak flows that could not be measured with current meters were measured using the slope-area method (Dalrymple and Benson, 1967). Daily mean values of streamflow were computed for eight stream-gaging stations (225800, 226000, 226200, 265600, 270900, 272200, $273950,275000)$. Data were computed using the streamflow measurements and water level or stage recorded at each of the stations as described by Rantz and others (1982).

\section{Suspended Sediment}

Suspended-sediment samples were collected at stream-gaging stations (table 2) using PS-69 automatic suspended-sediment samplers (Edwards and Glysson, 1988). Samples were collected once a day when conditions permitted, and more frequently during storms. Manual, depth-integrated cross-sectional samples were collected periodically using the equal-width-increment method (Edwards and Glysson, 1988) and were used to calibrate the PS-69 sample data (Porterfield, 1972). At station 265600, a Manning automatic sampler (Edwards and Glysson, 1988) was used at times in place of the PS69 because of suspended-sediment deposition near the PS-69 intake. In 1990, the automatic samplers at station 265600 were removed, and an observer was used to collect daily cross-sectional samples. Suspended-sediment concentrations (SSC) were determined by the U.S. Geological Survey office in Honolulu using methods described by Guy (1969). Suspended-sediment samples with concentrations of about $1,000 \mathrm{mg} / \mathrm{L}$ and higher were analyzed using method 2540D (Standard Methods for the Examination of Water and Wastewater, 19th ed., 1995) for total suspended solids (TSS) during water years 1995 through 1997. The difference in methods used to determine SSC and TSS results in a bias. TSS concentrations are lower compared with SSC when the percentage of sand-sized particles in the suspended-sediment sample increases beyond 25 percent (Gray and others, 2000). Based on particle-size analysis data collected during this study (table 4) and previously (Jones and others, 1971), average percentage of sand-sized particles did not exceed 20 percent. Therefore, the suspended-sediment samples that were computed for TSS concentrations during water years 1995 through 1997 were used without correction for bias. Suspendedsediment loads were computed using suspendedsediment concentrations and streamflow records as described by Porterfield (1972).

\section{Water Quality}

Water-quality measurements and samples were collected at the following 12 stream-gaging and waterquality stations: 226200, 227100, 265600, 265700, 266500, 267500, 269500, 270900, 272200, 273950, 274100 and 275000, as well as at various depths at 12 sites in Waimaluhia Reservoir (figs. 1, 2, and 5). Measurements of physical properties such as streamflow, water temperature, specific conductance, $\mathrm{pH}$, and dissolved oxygen were made in the field. In addition, samples were collected and subsequently analyzed for inorganic constituents such as turbidity, total suspended solids, major ions, nutrients, and trace metals, biological constituents such as fecal coliform bacteria, and organic constituents such as total organic carbon, oil and grease, pesticides, herbicides, and polychlorinated compounds. All physical measurements and samples for chemical constituent analyses were collected and processed according to guidelines in Ward and Harr (1990).

Stream sample collection was done using an equalwidth increment method when flow conditions (stream widths greater than $3 \mathrm{ft}$ and depths greater than $0.5 \mathrm{ft}$ ) permitted otherwise by dip sampling at the center of streamflow. Inorganic samples were collected using a DH-81 sampler with 1-liter polyethylene bottles when depths were greater than $0.5 \mathrm{ft}$. An open mouth polyethylene bottle was used to collect samples from shallower depths. A churn splitter (Ward and Harr, 1990) was used to composite and split all inorganic samples. Samples for fecal coliform bacteria and organic analyses were collected directly into sterile (bacteria) or baked 
(organic) glass containers by dip sampling at the center of streamflow.

Samples from Waimaluhia Reservoir were collected using a modified vertical configuration VanDorn sampler until 1995, after which a standard horizontal configuration Van-Dorn sampler was used (Ward and Harr, 1990). Reservoir measurements for physical properties and sampling for turbidity, total suspended solids, nutrients, and fecal coliform bacteria was done by depth at cross-sections 1-3 and 3-2 (fig. 5). In addition, at cross section 1-3, samples were collected by depth and then composited for analyses of major ions, trace metal, and organic constituents. All samples, except those for major ions and trace metals, were collected directly into sample bottles from the Van-Dorn sampler. Major ion and trace metal samples were composited first in a churn splitter. The composite for organic constituents at cross-section 1-3 was done by filling part of each bottle from part of the sample collected at the various depths. Measurements for physical properties only were done by depth at the remaining reservoir sites (fig. 5) and these data are not summarized in this report.

Water-quality samples at stream sites were collected on average ten times per year. Sampling dates were equally divided between wet (October to April) and dry (May to September) seasons. Measurements of streamflow, water temperature, specific conductance, $\mathrm{pH}$, and dissolved oxygen and samples for turbidity and total suspended solids were collected for all ten of the sampling dates during a water year. Samples for nutrients and fecal coliform bacteria were collected during four of the ten sampling dates, and samples for major ions, trace metals, and organic constituents were collected during two of the ten sampling dates. At Waimaluhia Reservoir, measurements of water temperature, specific conductance, $\mathrm{pH}$, and dissolved oxygen and samples for turbidity, nutrients, and fecal coliform bacteria were collected four times a year and samples for major ions, trace metals, and organic constituents were collected during two of the four sampling dates.

Starting in water year 1995, samples for inorganic constituents such as major ions, nutrients, and trace metals were collected and processed using the part per billion protocol in Horowitz and others (1994). Samples for trace metal analyses were preserved with non-ultrapure nitric acid until water year 1995 when the use of ultra-pure nitric acid was started. Prior to October 1994, samples for nutrient analyses were preserved with mercuric chloride and chilled to $4^{\circ} \mathrm{C}$. After October 1994, nutrient samples were preserved only by chilling. Starting in January 1999, nutrient samples for total ammonia and organic nitrogen and total phosphorus were preserved with sulfuric acid and chilled.

The methods used for the analyses of all the waterquality properties and constituents followed those in Wershaw and others (1987), Britton and Greeson (1989), Fishman and Friedman (1989), and Fishman (1993). All laboratory analyses were conducted by the U.S. Geological Survey's National Water Quality Laboratory (NWQL) in Colorado. Quality control practices at NWQL are documented in Friedman and Erdmann (1982) and Pritt and Rease (1992). In January 1993, laboratory analysis for total nitrate and nitrite was discontinued and only dissolved nitrate and nitrite were analyzed after that date, because values for total and dissolved nitrate and nitrite were statistically indistinguishable (U.S. Geological Survey Office of Water Quality Technical Memorandum 93.04).

\section{STATISTICAL SUMMARY OF HYDROLOGIC AND WATER-QUALITY DATA}

\section{Rainfall}

Total annual rainfall by water year for the five rain gages in the study area are shown in table 5. Compared to average rainfall for the 1983-99 period, 1988-89 and 1996-97 were relatively wet, whereas 1984, 1992, 1995, and 1998 were relatively dry. Figure 6 shows that rainfall was generally higher during the construction period in the Haiku drainage basin.

\section{Streamflow}

Total annual streamflow for the eight streamgaging stations in the study area are shown in table 6 . Total annual streamflow is determined by summing the daily mean values of discharge for the water year. Total annual streamflow was higher in water years 1988 , 1989, 1991, 1994, and 1997 than during the other years of the 1983-99 study period (table 6). Annual streamflow ranged from 122 cubic feet per second-days 


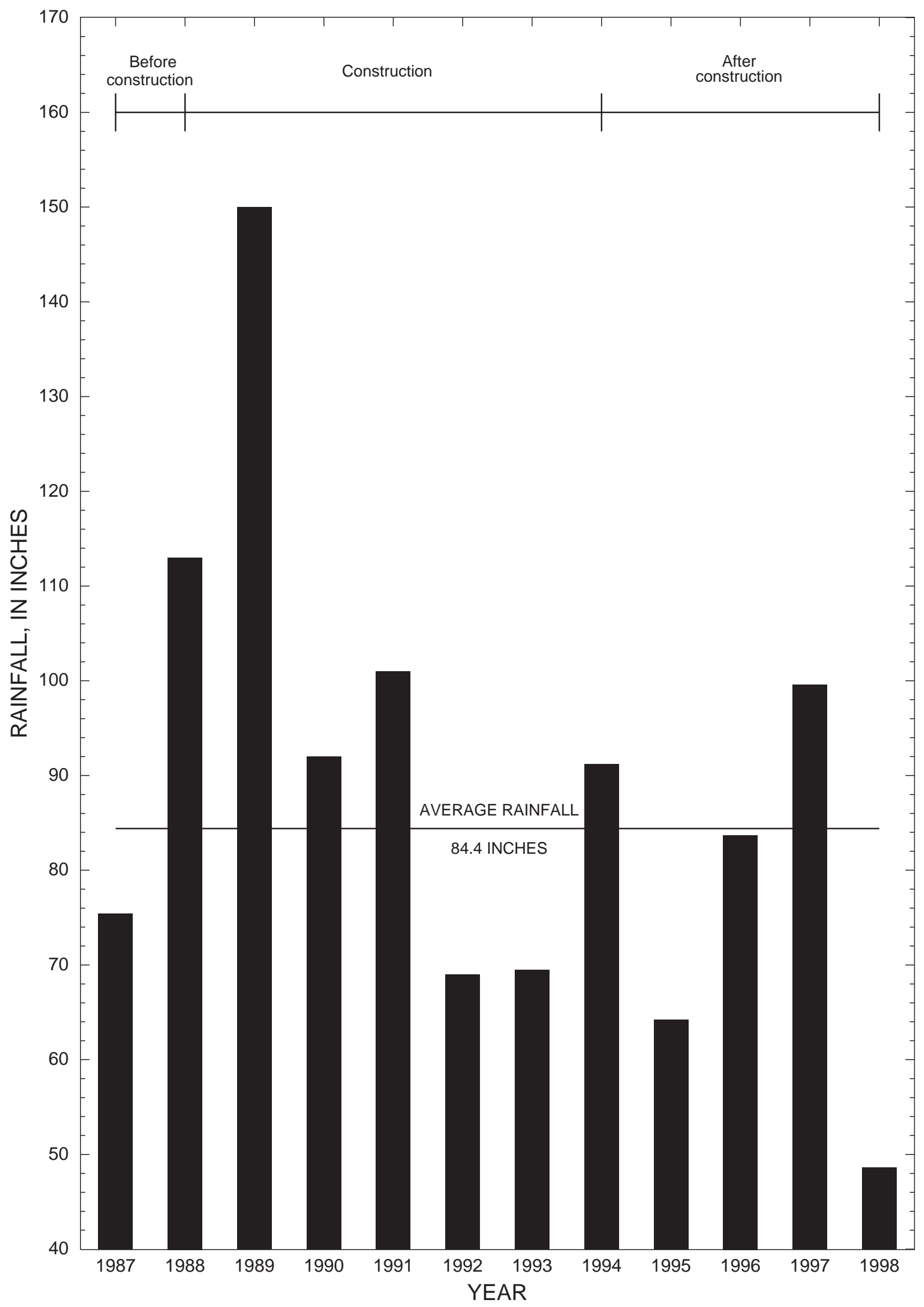

Figure 6. Total annual rainfall at Haiku rain gage 839.3, Oahu, Hawaii, water years 1987-98. 
[( $\left.\left.\mathrm{ft}^{3} / \mathrm{s}\right) \mathrm{d}\right]$ at station 265600 in 1984 to 6,070 at station 272200 in 1988 . Long-term records available for stream-gaging stations 226000, 272200, and 275000 prior to the 1983-99 period show that annual streamflow was higher in 1932 at station 226000 $\left(5,740\left[\left(\mathrm{ft}^{3} / \mathrm{s}\right) \mathrm{d}\right]\right), 1982$ at station $272200(8,040$ [(ft $3 / \mathrm{s}) \mathrm{d}])$, and in 1941 at station $275000(3,000$ $\left.\left[\left(\mathrm{ft}^{3} / \mathrm{s}\right) \mathrm{d}\right]\right)$ than for any year during the study period (table 6). The years of highest streamflow during the study period coincided with years of high rainfall (table 5). At North Halawa Stream station 226200, the years of highest streamflow corresponded to the period of highway construction in the valley (fig. 7). Average streamflow per unit of drainage basin area $\left(\left[\left(\mathrm{ft}^{3} / \mathrm{s}\right) \mathrm{d}\right] / \mathrm{mi}^{2}\right.$, table 7) can be used to compare streamflow between gages. Streamflow per unit of drainage basin area ranged from $110\left(\left[\left(\mathrm{ft}^{3} / \mathrm{s}\right) \mathrm{d}\right] / \mathrm{mi}^{2}\right)$ at station 265600 in 1984 to $2,620\left(\left[\left(\mathrm{ft}^{3} / \mathrm{s}\right) \mathrm{d}\right] / \mathrm{mi}^{2}\right)$ at station 273950 in 1989 (table 7).

\section{Suspended Sediment}

Suspended-sediment concentrations at seven stream-gaging stations $(225800,226200,265600$, $270900,272200,273950,275000)$ are shown in table 8. Suspended-sediment concentrations are summarized by water year and include both automatically and manually collected samples. Suspended-sediment concentrations ranged from less than $0.5 \mathrm{mg} / \mathrm{L}$ at most stations to 32,800 $\mathrm{mg} / \mathrm{L}$ at station 226200 in water year 1997 (table 8). Annual median suspended-sediment concentrations ranged from $1 \mathrm{mg} / \mathrm{L}$ at station 275000 in water years 1991 and 1997 to $1,320 \mathrm{mg} / \mathrm{L}$ at station 270900 in water year 1987 (table 8). Total annual suspended-sediment loads at the same seven stream-gaging stations are shown in table 9. Total annual suspended-sediment loads were variable from year to year and station to station. Total annual loads ranged from 23.7 tons at station 265600 in 1984 to 19,600 tons at station 226200 in 1997 (table 9). High annual loads correlated with years of high streamflow (tables 6 and 9). Total annual suspended-sediment loads per total annual streamflow (tons per cubic feet per second-day [tons/( $\left.\left.\mathrm{ft}^{3} / \mathrm{s}\right) \mathrm{d}\right]$ ) in table 10 can be used to compare total annual loads between water years at the same station. Loads per total annual streamflow ranged from 0.04 tons $/\left(\mathrm{ft}^{3} / \mathrm{s}\right) \mathrm{d}$ at station 272200 in 1984 to 7.26 tons $/\left(\mathrm{ft}^{3} / \mathrm{s}\right) \mathrm{d}$ at station
226200 in 1997 (table 10). Total annual suspendedsediment yields (total annual suspended-sediment loads per unit of drainage basin area) in tons per square mile (tons $/ \mathrm{mi}^{2}$ ) in table 11 can be used to compare suspended-sediment loads between stations. Sediment yields ranged from 7.71 tons $/ \mathrm{mi}^{2}$ in 1984 at station 226200 to 4,890 tons $/ \mathrm{mi}^{2}$ in 1997 also at station 226200 (table 11).

\section{Water Quality}

Statistical summaries that list the percentiles and minimum and maximum values for water-quality data from individual stream stations are given in tables 12 through 23 and summaries for the reservoir are given in tables 24 and 30. The water-quality data listed include selected physical properties, fecal coliform bacteria, organic carbon, oil and grease, major ions, nutrients, and trace metals. Statistical summaries at water-quality stations 265700, 266500, 267500, and 269500 only list physical properties, turbidity, total suspended solids, fecal coliform bacteria, and nutrients. Major ions, trace metals and organic compounds were sampled at these stations and at the wildlife pond on Hooleinaiwa Stream (figs. 2 and 5) in water years 1983-84 only. These data were summarized in Wong and Hill (1992) and are therefore not summarized in this report. Table 31 is a list of pesticides, herbicides, and polychlorinated compounds analyzed for during this study with corresponding laboratory detection limits. Only those compounds detected are listed in table 32. Properties or constituents had statistics computed only when the number of samples collected exceeded five. Summary statistics for data that include observations at or below laboratory reporting detection limits, called censored data, were computed by log-probability regression (Helsel and Gilliom, 1986). This method estimates summary statistics by combining above-reporting-limit values with extrapolated below-reporting-limit values. Some constituents had different laboratory reporting detection limits during the 17-year study period due to improving analytical techniques.

Statistical Summary of Hydrologic and Water-Quality Data 13 


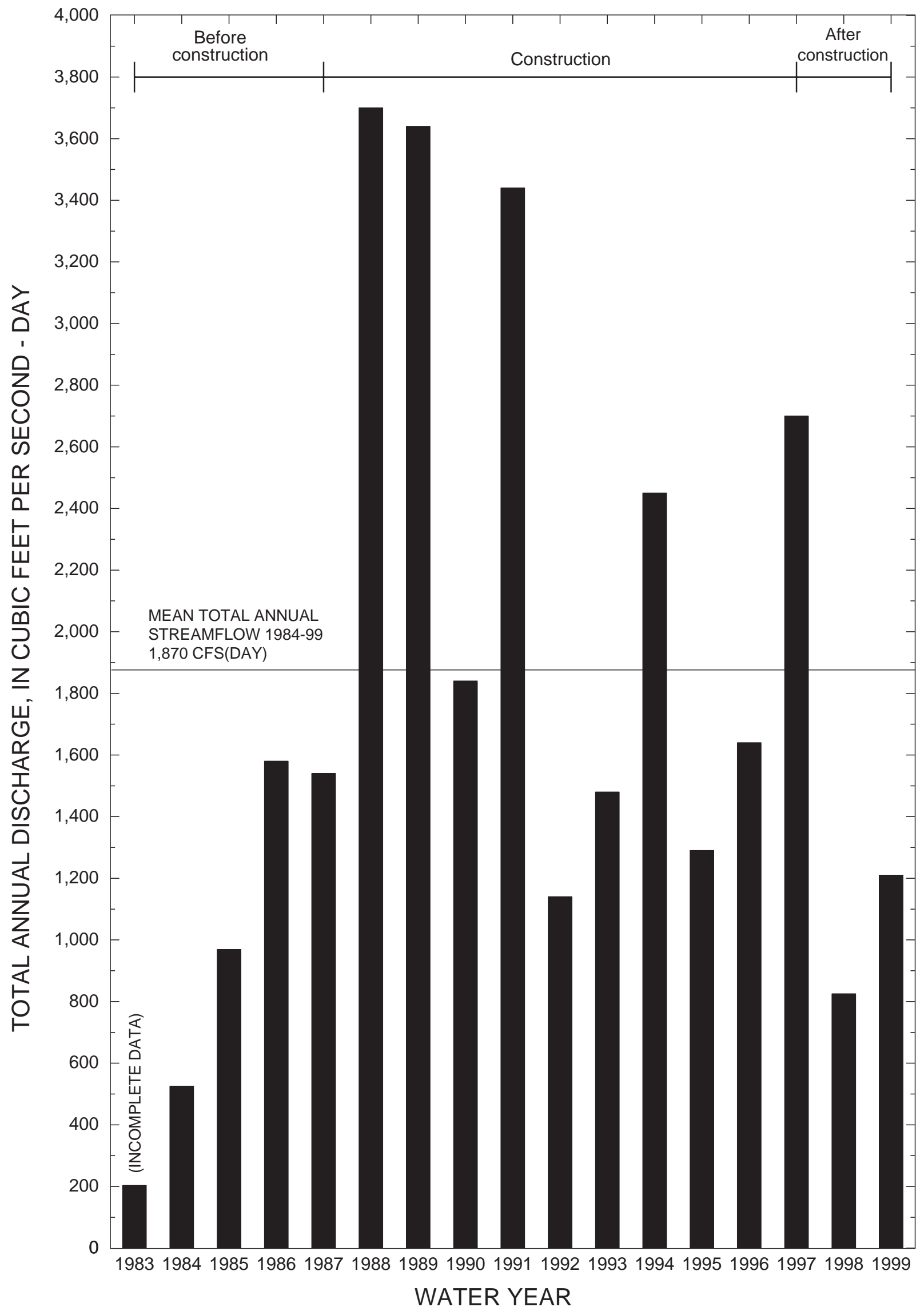

Figure 7. Total annual streamflow at station 16226200, North Halawa Stream near Honolulu, Oahu, Hawaii, water years 1983-99. 


\section{REFERENCES CITED}

Blumenstock, D.I., and Price, Saul, 1961, Climates of the states-Hawaii: U.S. Department of Commerce, Environmental Science Services Administration, Climatography of the United States no. 60-51, 27 p.

Britton, L.J., and Greeson, P.E., eds., 1989, Methods for collection and analysis of aquatic biological and microbiological samples: U.S. Geological Survey Techniques of Water-Resources Investigations, book 5, chap. A4, $363 \mathrm{p}$.

Dalrymple, Tate, and Benson, M.A., 1967, Measurement of peak discharge by the slope-area method: U.S. Geological Survey Techniques of Water-Resources Investigations, book 3, chap. A2, 12 p.

Edwards, T.K., and Glysson, G.D., 1988, Field methods for measurement of fluvial sediment: U.S. Geological Survey Open-File Report 86-531, 118 p.

Ekern, P.C., and Chang, Jen-Hu, 1985, Pan evaporation: State of Hawaii, 1894-1983: State of Hawaii, Department of Land and Natural Resources, Report R74, 172 p.

Fishman, M.J., and Friedman, L.C. eds., 1989, Methods for determination of inorganic substances in water and fluvial sediments ( $3 \mathrm{~d}$ ed.): U.S. Geological Survey Techniques of Water-Resources Investigations, book 5, chap. A1, 545 p.

Fishman, M.J., ed., 1993, Methods of analysis by the U.S. Geological Survey National Water Quality Laboratory-Determination of inorganic and organic constituents in water and fluvial sediments: U.S. Geological Survey Open-File Report 93-125, 217 p.

Foote, D.E., Hill, E.L., Nakamura, Sakuichi, and Stephens, Floyd, 1972, Soil survey of the islands of Kauai, Oahu, Maui, Molokai, and Lanai, State of Hawaii: U.S. Department of Agriculture, Soil Conservation Service, 232 p.

Friedman, L.C., and Erdmann, D.E., 1982, Quality assurance practices for the chemical and biological analyses of water and fluvial sediments: U.S. Geological Survey Techniques of Water Resources Investigations, book 5, chapter A6, $181 \mathrm{p}$.

Gray, J.R., Glysson, G.D., Turcios, L.M., and Schwarz, G.E., 2000, Comparability of suspended-sediment concentration and total suspended solids data: U.S. Geological Survey Water-Resources Investigations Report 00-4191, $14 \mathrm{p}$.

Guy, H.P., 1969, Laboratory theory and methods for sediment analysis: U.S. Geological Survey Techniques of Water-Resources Investigations, book 5, chap. C1, 58 p.

Helsel, D.R., and Gilliom, R.J., 1986, Estimation of distributional parameters for censored trace-level water quality data II. Verification and applications: Water Resources Research, v. 22, no. 2, p. 147-155.
Hill, B.R., Fontaine, R.A., Taogoshi, R.I., and Teeters, P.C., 2000, Water resources data, Hawaii and other Pacific areas, water year 1999, vol. 1, Hawaii: U.S. Geological Survey Water-Data Report HI-99-1, 339 p.

Hinds, N.E.A., 1925, Amphitheater valley heads: Journal of Geology, v. 33, p. 816-818.

Horowitz, A.J., Demas, C.R., Fitzgerald, K.K., Miller, T.L., and Rickert, D.R., 1994, U.S. Geological Survey protocol of the collection and processing of surface-water samples for the subsequent determination of inorganic constituents in filtered water: U.S. Geological Survey Open-File Report 94-539, 57 p.

Izuka, S.K., 1992, Geology and stream infiltration of North Halawa Valley, Oahu, Hawaii: U.S. Geological Survey Water-Resources Investigations Report 91-4197, 21 p.

Jones, B.L., Nakahara, R.H., and Chinn, S.S.W., 1971, Reconnaissance study of sediment transported by streams, island of Oahu: State of Hawaii, Department of Land and Natural Resources, Circular C33, 45 p.

Mink, J.F., 1960, Distribution pattern of rainfall in the leeward Koolau Mountains, Oahu, Hawaii: Journal of Geophysical Research, v. 65, no. 9, p. 2,869-2,876.

Pritt, J.W., and Raese, J.W., eds., 1992, Quality assurance/quality control manual National Water Quality Laboratory: U.S. Geological Survey Open-File Report 94-708, 26 p.

Porterfield, George, 1972, Computation of fluvial sediment discharge: U.S. Geological Survey Techniques of WaterResources Investigations, book 3, chap. C3, 66 p.

Rantz, S.E., and others, 1982, Measurement and computation of streamflow: Volume 1. Measurement of stage and discharge, Volume 2. Computation of discharge: U.S. Geological Survey Water-Supply Paper 2175, 284 p. and $346 \mathrm{p}$.

Searcy, J.K., 1959, Flow-duration curves: U.S. Geological Survey Water-Supply Paper 1542-A, Manual of Hydrology: Part 2. Low-Flow Techniques, 33 p.

Standard Methods for the Examination of Water and Wastewater, 19th ed., 1995, various pagination.

Takasaki, K.J., Hirashima, G.T., and Lubke, E.R., 1969, Water resources of windward Oahu, Hawaii: U.S. Geological Survey Water-Supply Paper 1894, 119 p.

U.S. Department of Transportation, Federal Highway Administration, and State of Hawaii Department of Transportation, 1987, Final third supplement to the Interstate Route H-3 Environmental Impact Statement, Interstate Route H-3 Halawa to Halekou Interchange, Report Number FHWA-HI-EIS-87-01-F(S), various pagination.

Visher, F.N., and Mink, J.F., 1964, Ground-water resources in Southern Oahu, Hawaii: U.S. Geological Survey Water-Supply Paper 1778, 133 p. 
Ward, J.R., and Harr, C.A., eds., 1990, Methods for collection and processing of surface-water and bed material samples for physical and chemical analyses: U.S. Geological Survey Open-File Report 90-140, 71 p.

Wentworth, C.K., 1943, Soil avalanches on Oahu, Hawaii: Bulletin of the Geological Society of America, v. 54, p. 53-64.

Wershaw, R.L., Fishman, M.J., Grabbe, R.R., Lowe, L.E., 1987, Methods for the determination of organic substances in water and fluvial sediments: U.S. Geological Survey Techniques of Water Resources Investigations, book 5 , chapter A3, $80 \mathrm{p}$.

Wong, M.F., and Hill, B.R., 1992, Statistical summary of hydrologic and water-quality data from the North Halawa, Haiku, and Kamooalii drainage basins, Oahu, Hawaii, water years 1983-89: U.S. Geological Survey Water-Resources Investigations Report 92-4049, 52 p. 
Table 1. Chronology of construction activities, H-3 Highway construction project, 1983-99, Oahu, Hawaii

[Locations of construction activities and stream-gaging stations are shown in figures 2-3; station numbers are abbreviated, complete numbers are preceded by 16; do., ditto. Start and end dates provided by the State of Hawaii, Department of Transportation, (written commun., 1999)]

\begin{tabular}{|c|c|c|}
\hline Construction activity & Start date & End date \\
\hline \multicolumn{3}{|c|}{ Halawa drainage basin—downstream stream-gaging station 227100} \\
\hline \multicolumn{3}{|c|}{ N. Halawa sub-drainage basin-downstream stream-gaging station $225800,226000,226200$, and 227100} \\
\hline 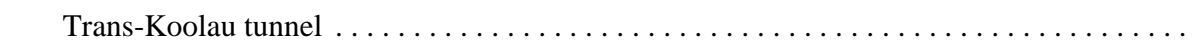 & 03/19/91 & $02 / 07 / 98^{2}$ \\
\hline 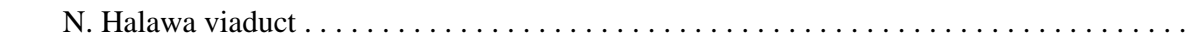 & $02 / 21 / 92$ & 06/95 \\
\hline \multicolumn{3}{|c|}{ N. Halawa sub-drainage basin—downstream stream-gaging stations 226000,226200 and 227100} \\
\hline 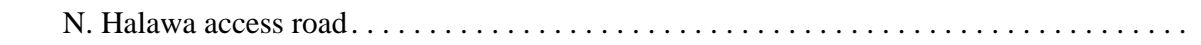 & $11 / 02 / 87$ & 03/89 \\
\hline Exploratory tunnel ... & $02 / 27 / 89$ & $03 / 90$ \\
\hline Drilled shaft test program & $11 / 13 / 90$ & 02/91 \\
\hline Trans-Koolau tunnel .... & 03/19/91 & $02 / 07 / 98^{2}$ \\
\hline N. Halawa viaduct . . . . . . . . . . . . & $02 / 21 / 92$ & 06/95 \\
\hline N. Halawa Valley highway Unit I Phase $1 \mathrm{~A} \ldots$ & $04 / 11 / 94$ & $09 / 95$ \\
\hline N. Halawa Valley highway Unit I Phase 1B . & $05 / 24 / 95$ & $12 / 12 / 97$ \\
\hline 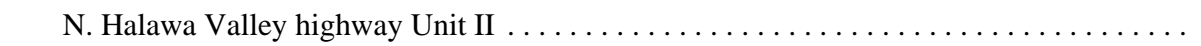 & 08/01/94 & $12 / 12 / 97$ \\
\hline \multicolumn{3}{|l|}{ Haiku drainage basin—downstream stream-gaging station 275000} \\
\hline Haiku access $\operatorname{road} \ldots \ldots \ldots \ldots \ldots \ldots \ldots \ldots \ldots \ldots \ldots \ldots \ldots \ldots \ldots \ldots \ldots$ & $10 / 24 / 88$ & $06 / 90$ \\
\hline Exploratory tunnel........ & $02 / 27 / 89$ & $03 / 90$ \\
\hline Haiku Valley bridges..... & 08/07/89 & $03 / 91$ \\
\hline Windward viaduct .... & 01/08/90 & $05 / 13 / 93$ \\
\hline Trans-Koolau tunnel . . . . . . . & $10 / 01 / 90$ & $02 / 07 / 98^{2}$ \\
\hline
\end{tabular}

Kaneohe drainage basin—downstream stream-gaging station 274100

S. Fork Kapunahala sub-drainage basin-downstream stream-gaging stations $\mathbf{2 7 3 9 5 0}$ and $\mathbf{2 7 4 1 0 0}$

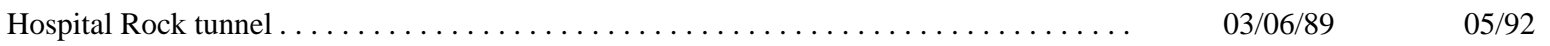

Kaneohe Interchange . . . . . . . . . . . . . . . . . . . . . . . . . . . . . . . . . 01/19/93 12/22/95

Kamooalii sub-drainage basin—downstream stations 265600, 265700, 266500, 267500, 269500, 270900, 272200, Waimaluhia Reservoir, and 274100

Windward highway $\ldots \ldots \ldots \ldots \ldots \ldots \ldots \ldots \ldots \ldots \ldots \ldots \ldots \ldots \ldots \ldots \ldots \ldots \ldots \ldots \ldots$ 06.19/89

Right Branch Kamooalii sub-drainage basin—downstream stations 265600, 272200, 274100, and Waimaluhia Reservoir

\begin{tabular}{|c|c|c|}
\hline Halekou Interchange ${ }^{1}$ & $02 / 22 / 83$ & $12 / 01 / 83$ \\
\hline$\ldots \ldots \ldots \ldots \ldots \ldots$ & $03 / 02 / 84$ & $07 / 31 / 85$ \\
\hline$\ldots \ldots \ldots \ldots$ & $11 / 04 / 85$ & $02 / 28 / 86$ \\
\hline do. ........ & $11 / 02 / 86$ & $12 / 31 / 86$ \\
\hline 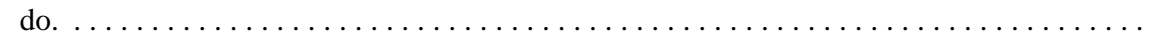 & $06 / 15 / 87$ & $09 / 30 / 88$ \\
\hline uku sub-drainage basin-downstream stream-gaging stations 270900,272200, and 2741 & & \\
\hline Windward highway ....... & 06/19/89 & $06 / 92$ \\
\hline 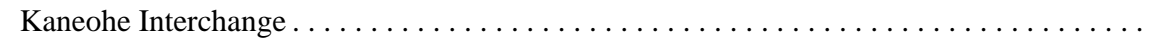 & $01 / 19 / 93$ & $12 / 22 / 95$ \\
\hline
\end{tabular}

${ }^{1}$ Work on Halekou Interchange interrupted multiple times by court injunctions

2 This is the official completion date for all work on the tunnel, however for the Haiku Valley portion of the tunnel construction, all land disturbance work was completed by 11/94, so for the purpose of determining the post-construction dates, 11/94 will be used. In North Halawa Valley, channel and earthwork near the tunnel continued until 09/97, so 09/30/97 will be used for the purpose of determining the post-construction dates. 
Table 2. Chronology of hydrologic and water-quality data-collection activities, H-3 Highway study area, Oahu, Hawaii

[--, no data; P, data collection continuous at present, 2001; station numbers are abbreviated, complete numbers are preceded by 16]

\begin{tabular}{|c|c|c|c|c|c|c|c|}
\hline \multirow{2}{*}{$\begin{array}{c}\text { Station } \\
\text { number or } \\
\text { site }\end{array}$} & \multirow[b]{2}{*}{ Station name } & \multicolumn{2}{|c|}{$\begin{array}{l}\text { Streamflow } \\
\text { data }\end{array}$} & \multicolumn{2}{|c|}{$\begin{array}{c}\text { Suspended-sediment } \\
\text { data }\end{array}$} & \multicolumn{2}{|c|}{$\begin{array}{l}\text { Water-quality } \\
\text { data }\end{array}$} \\
\hline & & Start & End & Start & End & Start & End \\
\hline \multicolumn{8}{|c|}{ Halawa drainage basin } \\
\hline 227100 & Halawa Str below $\mathrm{H}-1$ & -- & -- & -- & -- & Nov 1988 & Nov 1998 \\
\hline \multicolumn{8}{|c|}{ N. Halawa sub-drainage basin } \\
\hline 225800 & N. Halawa Str nr Kaneohe & Apr 1991 & Sept 1999 & Apr 1991 & Sept 1999 & -- & -- \\
\hline 226000 & N. Halawa Str nr Aiea & ${ }^{1}$ July 1953 & $\mathrm{P}$ & -- & -- & -- & -- \\
\hline 226200 & N. Halawa Str nr Honolulu & Feb 1983 & $\mathrm{P}$ & Feb 1983 & Sept 1999 & May 1983 & Mar 1999 \\
\hline \multicolumn{8}{|c|}{ Haiku drainage basin } \\
\hline 275000 & Haiku Str & ${ }^{3}$ Oct 1982 & $\mathrm{P}$ & ${ }^{4}$ July 1987 & Sept 1998 & Mar 1983 & Apr 1998 \\
\hline \multicolumn{8}{|c|}{ Kaneohe drainage basin } \\
\hline 274100 & Kaneohe Str & -- & -- & -- & -- & Nov 1988 & Aug 1999 \\
\hline \multicolumn{8}{|c|}{ S. Fork Kapunahala sub-drainage basin } \\
\hline 273950 & S. Fork Kapunahala Str & Oct 1987 & June 1998 & Oct 1987 & June 1998 & May 1988 & Apr 1998 \\
\hline \multicolumn{8}{|c|}{ Kamooalii sub-drainage basin } \\
\hline 265600 & R.B. Kamooalii Str & Feb 1983 & Sept 1997 & Feb 1983 & Sept 1997 & Feb 1983 & Jan 1998 \\
\hline 265700 & Kamooalii Str at alt $200 \mathrm{ft}$ & -- & -- & -- & -- & Feb 1983 & Jan 1998 \\
\hline 266500 & Hooleinaiwa Str at alt $220 \mathrm{ft}$ & -- & -- & -- & -- & Feb 1983 & Feb 1997 \\
\hline 267500 & $\begin{array}{l}\text { Hooleinaiwa Str abv conflu- } \\
\text { ence with Kamooalii Str }\end{array}$ & -- & -- & -- & -- & Feb 1983 & Jan 1998 \\
\hline 269500 & Kuou Str & -- & -- & -- & -- & Feb 1983 & Jan 1998 \\
\hline 270900 & Luluku Str & ${ }^{2}$ Apr 1984 & June 1998 & Apr 1984 & June 1998 & Feb 1983 & Apr 1998 \\
\hline 272200 & $\begin{array}{l}\text { Kamooalii Str below } \\
\text { Luluku Str }\end{array}$ & Nov 1976 & $\mathrm{P}$ & Nov 1976 & Sept 1998 & Feb 1983 & Apr 1998 \\
\hline \multicolumn{2}{|c|}{ Waimaluhia Reservoir } & -- & -- & -- & -- & May 1983 & Jan 1998 \\
\hline
\end{tabular}

${ }^{1}$ Streamflow data were previously collected $1929-33$

2 Streamflow data were previously collected 1960-63 (low flows only), 1965-71, 1971-84 (annual maximum only)

${ }^{3}$ Streamflow data were previously collected 1914-19, 1939-77

${ }^{4}$ Suspended-sediment data were previously collected December 1983 to September 1984 
Table 3. Physiography of basins upstream from gaging stations in the H-3 Highway study area, Oahu, Hawaii

[Basin relief is the maximum altitude in the basin minus the altitude at the gage. Channel length is the length of the longest stream in the basin selected from those shown as blue lines on the 1983 Oahu 1:24,000 topographic maps, and measured from the drainage basin divide to the gage. Channel gradient is the basin relief divided by the channel length; $\mathrm{ft}$, feet; $\mathrm{mi}^{2}$, square mile; $\mathrm{mi}$, mile; ft/ft, feet/foot]

\begin{tabular}{|c|c|c|c|c|c|c|}
\hline $\begin{array}{l}\text { Station } \\
\text { number or } \\
\text { site }\end{array}$ & Station name & $\begin{array}{l}\text { Altitude of } \\
\text { gaging } \\
\text { station (ft) }\end{array}$ & $\begin{array}{l}\text { Basin relief } \\
\text { (ft) }\end{array}$ & $\begin{array}{c}\text { Drainage } \\
\text { area } \\
\left(\mathrm{mi}^{2}\right)\end{array}$ & $\begin{array}{l}\text { Channel } \\
\text { length } \\
\text { (mi) }\end{array}$ & $\begin{array}{c}\text { Channel } \\
\text { gradient } \\
\text { (ft/ft) }\end{array}$ \\
\hline 225800 & N. Halawa Str nr Kaneohe & 646 & 2,180 & 1.64 & 2.74 & 0.151 \\
\hline 226000 & N. Halawa Str nr Aiea & 320 & 2,510 & 3.45 & 5.55 & 0.086 \\
\hline 226200 & N. Halawa Str nr Honolulu & 160 & 2,670 & 4.01 & 6.59 & 0.077 \\
\hline 227100 & Halawa Str below H-1 & 20 & 2,800 & 8.90 & 8.99 & 0.059 \\
\hline 265600 & R.B. Kamooalii Str & 195 & 2,200 & 1.11 & 1.75 & 0.238 \\
\hline 265700 & Kamooalii Str at alt $200 \mathrm{ft}$ & 200 & 1,800 & 0.46 & 1.14 & 0.299 \\
\hline 266500 & Hooleinaiwa Str at alt $220 \mathrm{ft}$ & 220 & 2,480 & 0.41 & 0.91 & 0.516 \\
\hline 267500 & $\begin{array}{l}\text { Hooleinaiwa Str abv conf with } \\
\text { Kamooalii Str }\end{array}$ & 180 & 2,520 & 0.57 & 1.10 & 0.434 \\
\hline 269500 & Kuou Str & 220 & 2,460 & 0.34 & 0.91 & 0.512 \\
\hline 270900 & Luluku Str & 220 & 2,600 & 0.44 & 1.01 & 0.486 \\
\hline 272200 & Kamooalii Str below Luluku Str & 116 & 2,700 & 3.81 & 5.44 & 0.094 \\
\hline 273950 & S. Fork Kapunahala Str & 111 & 2,330 & 0.40 & 1.12 & 0.394 \\
\hline 274100 & Kaneohe Str & 40 & 2,780 & 5.22 & 7.05 & 0.075 \\
\hline 275000 & Haiku Str & 272 & 2,450 & 0.97 & 1.19 & 0.390 \\
\hline \multicolumn{2}{|c|}{ Waimaluhia Reservoir at outlet } & 160 & 2,540 & 3.20 & 5.18 & 0.093 \\
\hline
\end{tabular}


Table 4. Average suspended-sediment particle-size analyses at stream-gaging stations in the $\mathrm{H}-3$ Highway study area, Oahu, Hawaii, water years 1983-99

[station numbers are abbreviated, complete numbers are preceded by $16 ;--$, no data]

\begin{tabular}{cccc}
\hline Station & $\begin{array}{c}\text { Number of } \\
\text { number }\end{array}$ & $\begin{array}{c}\text { Average } \\
\text { sercentage of silt } \\
\text { and clay } \\
\text { particles }\end{array}$ & $\begin{array}{c}\text { Average } \\
\text { percentage of } \\
\text { sand-sized or } \\
\text { larger } \\
\text { particles }\end{array}$ \\
\hline 225800 & 18 & 95 & 5 \\
226200 & 41 & 86 & 14 \\
265600 & 1 & 68 & 32 \\
270900 & 8 & 77 & 23 \\
272200 & 8 & 79 & 21 \\
273950 & 0 & -- & -- \\
275000 & 10 & 86 & 14 \\
\hline Average of all stations & 82 & 18 \\
\hline
\end{tabular}

Table 5. Total annual rainfall in the H-3 Highway study area, Oahu, Hawaii, water years 1983-99

[All values in inches; --, no data; P, partial year of record, more than one month of record missing; e, estimated annual total, one or more days were estimated, data were estimated by comparison with nearby raingages; USGS station numbers are abbreviated, complete numbers are preceded by 16 ; Numbers below gage numbers or names are 4 digit State key numbers assigned to rain gages in Hawaii by the Commission on Water Resource Management, Department of Land and Natural Resources, State of Hawaii; average is for complete years only for the period 1983-99]

\begin{tabular}{|c|c|c|c|c|c|}
\hline \multirow[b]{2}{*}{ Water year } & \multicolumn{5}{|c|}{ Rain gage } \\
\hline & $\begin{array}{l}226000 \\
(772.1)\end{array}$ & $\begin{array}{l}226200 \\
(771.9)\end{array}$ & $\begin{array}{l}265600 \\
(781.9)\end{array}$ & $\begin{array}{l}270900 \\
(781.11)\end{array}$ & $\begin{array}{c}\text { Haiku } \\
(839.3)\end{array}$ \\
\hline 1983 & 65.4 & $\mathrm{P}$ & $\mathrm{P}$ & -- & -- \\
\hline 1984 & 44.1 & 23.5 & 38.9 & $\mathrm{P}$ & -- \\
\hline 1985 & 61.6 & 40.7 & 63.9 & 66.8 & -- \\
\hline 1986 & 60.9 & 32.9 & 59.2 & 66.2 & $\mathrm{P}$ \\
\hline 1987 & 68.3 & 33.8 & 67.2 & 66.7 & 75.4 \\
\hline 1988 & 90.6 & 56.5 & 86.8 & 95.1 & 113 \\
\hline 1989 & e91.2 & 55.5 & 84.1 & 87.2 & 105 \\
\hline 1990 & 66.5 & 35.3 & 62.4 & 62.8 & 92.0 \\
\hline 1991 & 80.2 & 41.2 & 68.5 & 64.9 & e101 \\
\hline 1992 & 52.8 & 27.3 & 42.2 & e55.9 & 69.0 \\
\hline 1993 & e58.7 & e33.5 & $\mathrm{P}$ & 61.6 & 69.5 \\
\hline 1994 & $\mathrm{P}$ & 23.6 & $\mathrm{P}$ & 71.2 & 91.2 \\
\hline 1995 & 49.4 & e43.8 & e 40.2 & 49.1 & 64.2 \\
\hline 1996 & 53.7 & 43.4 & e60.1 & 73.7 & 83.7 \\
\hline 1997 & 63.8 & 61.0 & e75.9 & e81.5 & 99.6 \\
\hline 1998 & 33.3 & 23.7 & $\mathrm{P}$ & 44.7 & 48.6 \\
\hline 1999 & 35.9 & 31.8 & -- & -- & -- \\
\hline Average & 61.0 & 38.0 & 62.4 & 67.7 & 84.4 \\
\hline
\end{tabular}


Table 6. Total annual streamflow at stream-gaging stations in the H-3 Highway study area, Oahu, Hawaii, water years $1983-99$ [All values are in cubic feet per second-days ([ $\left.\left.\mathrm{ft}^{3} / \mathrm{s}\right] \mathrm{d}\right)$; --, no data; station numbers are abbreviated, complete numbers are preceded by 16]

\begin{tabular}{rrrrrrrrr}
\hline \multirow{2}{*}{$\begin{array}{c}\text { Water } \\
\text { year }\end{array}$} & $\mathbf{2 2 5 8 0 0}$ & $\mathbf{2 2 6 0 0 0}$ & $\mathbf{2 2 6 2 0 0}$ & $\mathbf{2 6 5 6 0 0}$ & $\mathbf{2 7 0 9 0 0}$ & $\mathbf{2 7 2 2 0 0}$ & $\mathbf{2 7 3 9 5 0}$ & $\mathbf{2 7 5 0 0 0}$ \\
\hline 1983 & -- & 964 & 1203 & 1267 & -- & 4,090 & -- & 877 \\
1984 & -- & 517 & 525 & 122 & 244.5 & 1,600 & -- & 520 \\
1985 & -- & 827 & 969 & 284 & 152 & 2,290 & -- & 604 \\
1986 & -- & 1,590 & 1,580 & 406 & 150 & 2,940 & -- & 674 \\
1987 & -- & 1,440 & 1,540 & 612 & 327 & 3,760 & -- & 790 \\
1988 & -- & 3,140 & 3,700 & 986 & 509 & 6,070 & 1,020 & 1,270 \\
1989 & -- & 3,040 & 3,640 & 1,040 & 532 & 5,930 & 1,050 & 1,420 \\
1990 & -- & 1,670 & 1,840 & 532 & 488 & 4,280 & 847 & 888 \\
1991 & 2458 & 2,740 & 3,440 & 762 & 737 & 5,000 & 938 & 1,230 \\
1992 & 818 & 1,030 & 1,140 & 308 & 471 & 3,030 & 828 & 806 \\
1993 & 1,020 & 1,410 & 1,480 & 605 & 394 & 3,610 & 860 & 738 \\
1994 & 1,560 & 2,150 & 2,450 & 609 & 519 & 4,240 & 838 & 941 \\
1995 & 708 & 1,030 & 1,290 & 216 & 606 & 2,600 & 704 & 618 \\
1996 & 801 & 1,260 & 1,640 & 504 & 520 & 3,580 & 722 & 735 \\
1997 & 1,200 & 1,890 & 2,700 & 721 & 554 & 5,370 & 894 & 1,030 \\
1998 & 506 & 609 & 824 & -- & 3341 & 2,730 & 3540 & 696 \\
1999 & 565 & 1,130 & 1,220 & -- & -- & 1,800 & -- & 566 \\
\hline
\end{tabular}

${ }^{1}$ Data from February to September

2 Data from April to September

${ }^{3}$ Data from October to June 
Table 7. Total annual streamflow per unit area at stream-gaging stations in the H-3 Highway study area, Oahu, Hawaii, water years 1983-99

[All values are in cubic feet per second-days per square mile of drainage basin area $\left(\left[\left(\mathrm{ft}^{3} / \mathrm{s}\right) \mathrm{d}\right] / \mathrm{mi}^{2}\right)$; only data for water years with complete streamflow records are listed; --, no data; station numbers are abbreviated, complete numbers are preceded by 16]

\begin{tabular}{|c|c|c|c|c|c|c|c|c|}
\hline \multirow{2}{*}{$\begin{array}{l}\text { Water } \\
\text { year }\end{array}$} & \multicolumn{8}{|c|}{ Station } \\
\hline & 225800 & 226000 & 226200 & 265600 & 270900 & 272200 & 273950 & 275000 \\
\hline 1983 & -- & 279 & -- & -- & -- & 1,070 & -- & 904 \\
\hline 1984 & -- & 150 & 131 & 110 & -- & 420 & -- & 536 \\
\hline 1985 & -- & 240 & 242 & 256 & 345 & 601 & -- & 623 \\
\hline 1986 & -- & 461 & 394 & 366 & 341 & 772 & -- & 695 \\
\hline 1987 & -- & 417 & 384 & 551 & 743 & 987 & -- & 814 \\
\hline 1988 & -- & 910 & 923 & 888 & 1,160 & 1,590 & 2,550 & 1,310 \\
\hline 1989 & -- & 881 & 908 & 937 & 1,210 & 1,560 & 2,620 & 1,460 \\
\hline 1990 & -- & 484 & 459 & 479 & 1,110 & 1,120 & 2,120 & 916 \\
\hline 1991 & -- & 794 & 858 & 686 & 1,680 & 1,310 & 2,340 & 1,270 \\
\hline 1992 & 499 & 299 & 283 & 277 & 1,070 & 795 & 2,070 & 831 \\
\hline 1993 & 622 & 409 & 369 & 545 & 895 & 948 & 2,150 & 761 \\
\hline 1994 & 951 & 623 & 611 & 549 & 1,180 & 1,110 & 2,100 & 970 \\
\hline 1995 & 432 & 299 & 323 & 195 & 1,380 & 682 & 1,760 & 637 \\
\hline 1996 & 488 & 365 & 409 & 454 & 1,180 & 940 & 1,810 & 758 \\
\hline 1997 & 732 & 548 & 674 & 650 & 1,260 & 1,410 & 2,240 & 1,060 \\
\hline 1998 & 308 & 177 & 205 & -- & -- & 717 & -- & 718 \\
\hline 1999 & 344 & 354 & 302 & -- & -- & 472 & -- & 584 \\
\hline
\end{tabular}


Table 8. Summary of suspended-sediment concentrations at stream-gaging stations in the H-3 Highway study area, Oahu, Hawaii, water years 1983-99

[All concentrations are reported in milligrams per liter; <, less than; unpublished data in the files of the U.S. Geological Survey, Hawaii District; station numbers are abbreviated, complete numbers are preceded by 16 ; d, during construction; $a$, after construction; b, before construction]

\begin{tabular}{|c|c|c|c|c|c|c|c|c|}
\hline \multirow{2}{*}{$\begin{array}{l}\text { Station } \\
\text { number }\end{array}$} & \multirow{2}{*}{$\begin{array}{l}\text { Water } \\
\text { year }\end{array}$} & \multirow{2}{*}{$\begin{array}{l}\text { Number of } \\
\text { samples }\end{array}$} & \multirow[b]{2}{*}{ Minimum } & \multicolumn{3}{|c|}{ Concentration percentile } & \multirow[b]{2}{*}{ Maximum } & \multirow[b]{2}{*}{ Notes } \\
\hline & & & & 25 & 50 & 75 & & \\
\hline \multirow[t]{9}{*}{225800} & 1991 & 53 & $<0.5$ & 2 & 8 & 40 & 2,130 & $\mathrm{~d}$ \\
\hline & 1992 & 247 & $<0.5$ & 12 & 109 & 454 & 7,850 & $\mathrm{~d}$ \\
\hline & 1993 & 97 & 9 & 40 & 105 & 824 & 23,600 & $\mathrm{~d}$ \\
\hline & 1994 & 54 & 4 & 184 & 290 & 475 & 3,900 & $d$ \\
\hline & 1995 & 93 & 1 & 80 & 137 & 269 & 4,880 & $\mathrm{~d}$ \\
\hline & 1996 & 62 & 2 & 33 & 94 & 135 & 400 & $\mathrm{~d}$ \\
\hline & 1997 & 93 & $<0.5$ & 12 & 50 & 129 & 742 & $\mathrm{~d}$ \\
\hline & 1998 & 50 & $<0.5$ & 5 & 14 & 85 & 3,160 & $\mathrm{a}$ \\
\hline & 1999 & 146 & $<0.5$ & 3 & 44 & 118 & 1,740 & $\mathrm{a}$ \\
\hline \multirow[t]{17}{*}{226200} & 1983 & 45 & $<0.5$ & 3 & 6 & 17 & 79 & $\mathrm{~b}$ \\
\hline & 1984 & 90 & 1 & 4 & 7 & 23 & 144 & $\mathrm{~b}$ \\
\hline & 1985 & 83 & 2 & 6 & 13 & 101 & 2,800 & $\mathrm{~b}$ \\
\hline & 1986 & 153 & 1 & 3 & 5 & 18 & 1,880 & $\mathrm{~b}$ \\
\hline & 1987 & 198 & 1 & 3 & 7 & 30 & 4,740 & $\mathrm{~b}$ \\
\hline & 1988 & 420 & $<0.5$ & 13 & 82 & 294 & 6,250 & d \\
\hline & 1989 & 300 & 1 & 47 & 324 & 1,150 & 18,000 & $\mathrm{~d}$ \\
\hline & 1990 & 375 & $<0.5$ & 6 & 25 & 197 & 4,940 & $d$ \\
\hline & 1991 & 466 & $<0.5$ & 19 & 70 & 228 & 8,330 & d \\
\hline & 1992 & 158 & 3 & 31 & 461 & 1,270 & 15,200 & d \\
\hline & 1993 & 244 & 3 & 37 & 135 & 649 & 15,300 & d \\
\hline & 1994 & 263 & 5 & 24 & 56 & 229 & 5,990 & d \\
\hline & 1995 & 253 & 4 & 18 & 49 & 222 & 5,930 & d \\
\hline & 1996 & 211 & 6 & 27 & 244 & 783 & 9,650 & d \\
\hline & 1997 & 279 & $<0.5$ & 7 & 28 & 218 & 32,800 & d \\
\hline & 1998 & 287 & $<0.5$ & 2 & 4 & 19 & 13,000 & $\mathrm{a}$ \\
\hline & 1999 & 451 & $<0.5$ & 3 & 11 & 69 & 2,360 & $\mathrm{a}$ \\
\hline \multirow[t]{15}{*}{265600} & 1983 & 328 & 3 & 11 & 17 & 51 & 8,950 & d \\
\hline & 1984 & 243 & 3 & 6 & 9 & 22 & 6,340 & d \\
\hline & 1985 & 424 & 2 & 6 & 9 & 17 & 5,080 & d \\
\hline & 1986 & 120 & 7 & 84 & 160 & 316 & 1,670 & d \\
\hline & 1987 & 96 & 7 & 11 & 115 & 376 & 3,400 & $\mathrm{~d}$ \\
\hline & 1988 & 266 & 1 & 56 & 254 & 561 & 6,040 & d \\
\hline & 1989 & 131 & 18 & 137 & 180 & 281 & 13,000 & d \\
\hline & 1990 & 292 & 1 & 8 & 20 & 240 & 12,500 & d \\
\hline & 1991 & 369 & 2 & 5 & 7 & 16 & 1,800 & d \\
\hline & 1992 & 385 & $<0.5$ & 2 & 3 & 4 & 174 & $d$ \\
\hline & 1993 & 389 & $<0.5$ & 2 & 2 & 4 & 1,440 & $\mathrm{a}$ \\
\hline & 1994 & 363 & $<0.5$ & 2 & 2 & 4 & 324 & $\mathrm{a}$ \\
\hline & 1995 & 240 & 1 & 2 & 3 & 4 & 104 & $\mathrm{a}$ \\
\hline & 1996 & 18 & $<0.5$ & 2 & 8 & 24 & 44 & $\mathrm{a}$ \\
\hline & 1997 & 39 & $<0.5$ & 2 & 4 & 27 & 300 & $\mathrm{a}$ \\
\hline \multirow[t]{6}{*}{270900} & 1984 & 31 & 6 & 15 & 54 & 417 & 1,450 & $\mathrm{~b}$ \\
\hline & 1985 & 127 & 2 & 17 & 47 & 207 & 3,060 & $\mathrm{~b}$ \\
\hline & 1986 & 52 & 6 & 15 & 66 & 615 & 6,480 & $\mathrm{~b}$ \\
\hline & 1987 & 62 & 10 & 270 & 1,320 & 2,320 & 4,640 & $\mathrm{~b}$ \\
\hline & 1988 & 231 & 1 & 88 & 267 & 601 & 5,980 & $\mathrm{~b}$ \\
\hline & 1989 & 195 & 6 & 90 & 220 & 686 & 8,500 & $\mathrm{~b}$ \\
\hline
\end{tabular}


Table 8. Summary of suspended-sediment concentrations at stream-gaging stations in the H-3 Highway study area, Oahu, Hawaii, water years 1983-99--Continued

[All concentrations are reported in milligrams per liter; <, less than; unpublished data in the files of the U.S. Geological Survey, Hawaii District; station numbers are abbreviated, complete numbers are preceded by 16 ; d, during construction; a, after construction; $b$, before construction]

\begin{tabular}{|c|c|c|c|c|c|c|c|c|}
\hline \multirow{2}{*}{$\begin{array}{l}\text { Station } \\
\text { number }\end{array}$} & \multirow{2}{*}{$\begin{array}{l}\text { Water } \\
\text { year }\end{array}$} & \multirow{2}{*}{$\begin{array}{l}\text { Number of } \\
\text { samples }\end{array}$} & \multirow[b]{2}{*}{ Minimum } & \multicolumn{3}{|c|}{ Concentration percentile } & \multirow[b]{2}{*}{ Maximum } & \multirow[b]{2}{*}{ Notes } \\
\hline & & & & 25 & 50 & 75 & & \\
\hline \multicolumn{9}{|c|}{ 270900--Continued } \\
\hline & 1990 & 105 & $<0.5$ & 10 & 41 & 170 & 2,930 & d \\
\hline & 1991 & 440 & $<0.5$ & 9 & 36 & 147 & 3,250 & $\mathrm{~d}$ \\
\hline & 1992 & 82 & 2 & 24 & 96 & 253 & 1,960 & $\mathrm{~d}$ \\
\hline & 1993 & 85 & 4 & 18 & 69 & 192 & 2,650 & d \\
\hline & 1994 & 214 & 3 & 8 & 17 & 112 & 4,500 & d \\
\hline & 1995 & 161 & $<0.5$ & 5 & 8 & 15 & 2,270 & d \\
\hline & 1996 & 145 & 3 & 8 & 16 & 120 & 2,180 & $\mathrm{a}$ \\
\hline & 1997 & 198 & 1 & 5 & 16 & 70 & 1,710 & $\mathrm{a}$ \\
\hline & 1998 & 175 & $<0.5$ & 1 & 7 & 33 & 718 & $\mathrm{a}$ \\
\hline \multirow[t]{16}{*}{272200} & 1983 & 411 & 1 & 5 & 8 & 12 & 939 & $\mathrm{~d}$ \\
\hline & 1984 & 250 & 2 & 7 & 10 & 15 & 426 & $\mathrm{~d}$ \\
\hline & 1985 & 243 & 3 & 9 & 19 & 46 & 959 & $\mathrm{~d}$ \\
\hline & 1986 & 390 & 3 & 6 & 10 & 19 & 2,590 & d \\
\hline & 1987 & 451 & 2 & 5 & 8 & 19 & 4,290 & $\mathrm{~d}$ \\
\hline & 1988 & 253 & $<0.5$ & 5 & 18 & 55 & 2,390 & $\mathrm{~d}$ \\
\hline & 1989 & 287 & 3 & 10 & 22 & 49 & 2,670 & $\mathrm{~d}$ \\
\hline & 1990 & 363 & 1 & 7 & 12 & 27 & 8,400 & d \\
\hline & 1991 & 408 & 1 & 7 & 13 & 35 & 7,840 & d \\
\hline & 1992 & 240 & 3 & 6 & 9 & 13 & 191 & $\mathrm{~d}$ \\
\hline & 1993 & 217 & 1 & 6 & 8 & 18 & 1,130 & d \\
\hline & 1994 & 346 & 1 & 5 & 8 & 15 & 1,600 & $\mathrm{~d}$ \\
\hline & 1995 & 207 & 4 & 9 & 13 & 18 & 1,530 & d \\
\hline & 1996 & 204 & 1 & 4 & 7 & 16 & 1,150 & $\mathrm{a}$ \\
\hline & 1997 & 261 & 1 & 5 & 8 & 18 & 696 & $\mathrm{a}$ \\
\hline & 1998 & 91 & 2 & 7 & 11 & 29 & 357 & $\mathrm{a}$ \\
\hline \multirow[t]{11}{*}{273950} & 1988 & 280 & 3 & 9 & 19 & 169 & 1,470 & $\mathrm{~b}$ \\
\hline & 1989 & 150 & 7 & 33 & 340 & 964 & 9,870 & $\mathrm{~b}$ \\
\hline & 1990 & 145 & 4 & 9 & 13 & 103 & 1,200 & $\mathrm{~d}$ \\
\hline & 1991 & 97 & 15 & 108 & 609 & 1,740 & 8,570 & $\mathrm{~d}$ \\
\hline & 1992 & 35 & 19 & 35 & 89 & 229 & 1,730 & d \\
\hline & 1993 & 49 & 8 & 22 & 46 & 152 & 2,020 & d \\
\hline & 1994 & 166 & 5 & 12 & 18 & 174 & 6,270 & d \\
\hline & 1995 & 315 & 2 & 8 & 11 & 18 & 656 & $\mathrm{~d}$ \\
\hline & 1996 & 111 & 6 & 26 & 65 & 144 & 614 & $\mathrm{a}$ \\
\hline & 1997 & 100 & 7 & 26 & 40 & 84 & 794 & $\mathrm{a}$ \\
\hline & 1998 & 54 & 6 & 11 & 16 & 84 & 417 & $\mathrm{a}$ \\
\hline \multirow[t]{12}{*}{275000} & 1984 & 113 & 1 & 3 & 5 & 7 & 81 & $\mathrm{~b}$ \\
\hline & 1988 & 237 & 1 & 4 & 7 & 16 & 3,430 & $\mathrm{~b}$ \\
\hline & 1989 & 370 & 1 & 5 & 13 & 36 & 14,000 & d \\
\hline & 1990 & 273 & $<0.5$ & 3 & 6 & 25 & 15,400 & d \\
\hline & 1991 & 360 & $<0.5$ & 1 & 1 & 11 & 15,700 & $\mathrm{~d}$ \\
\hline & 1992 & 382 & $<0.5$ & 2 & 4 & 8 & 15,780 & d \\
\hline & 1993 & 255 & 1 & 3 & 5 & 13 & 3,260 & d \\
\hline & 1994 & 341 & 1 & 4 & 6 & 16 & 7,090 & d \\
\hline & 1995 & 193 & 1 & 3 & 5 & 8 & 4,030 & $\mathrm{a}$ \\
\hline & 1996 & 147 & 1 & 3 & 5 & 14 & 6,610 & $\mathrm{a}$ \\
\hline & 1997 & 100 & $<0.5$ & 1 & 1 & 14 & 785 & $\mathrm{a}$ \\
\hline & 1998 & 212 & $<0.5$ & 1 & 2 & 3 & 86 & $\mathrm{a}$ \\
\hline
\end{tabular}


Table 9. Total annual suspended-sediment loads at stream-gaging stations in the H-3 Highway study area, Oahu, Hawaii, water years 1983-99

[All values are in tons; --, no data; station numbers are abbreviated, complete numbers are preceded by 16]

\begin{tabular}{|c|c|c|c|c|c|c|c|}
\hline \multirow{2}{*}{$\begin{array}{l}\text { Water } \\
\text { year }\end{array}$} & \multicolumn{7}{|c|}{ Station number } \\
\hline & 225800 & 226200 & 265600 & 270900 & 272200 & 273950 & 275000 \\
\hline 1983 & -- & 15.45 & ${ }^{1} 12.7$ & -- & 173 & -- & -- \\
\hline 1984 & -- & 30.9 & 23.7 & ${ }^{2} 1.37$ & 76.6 & -- & ${ }^{3} 6.08$ \\
\hline 1985 & -- & 314 & 541 & 43.0 & 336 & -- & -- \\
\hline 1986 & -- & 362 & 472 & 91.1 & 322 & -- & -- \\
\hline 1987 & -- & 615 & 631 & 402 & 1,190 & -- & ${ }^{4} 114$ \\
\hline 1988 & -- & 3,500 & 1,730 & 485 & 2,240 & 187 & 1,310 \\
\hline 1989 & -- & 16,600 & 1,220 & 180 & 1,290 & 488 & 3,400 \\
\hline 1990 & -- & 2,260 & 1,160 & 52.6 & 593 & 73.5 & 1,660 \\
\hline 1991 & 2894 & 5,220 & 512 & 333 & 1,270 & 435 & 2,970 \\
\hline 1992 & 3,650 & 4,290 & 64.8 & 70.4 & 148 & 81.6 & 1,610 \\
\hline 1993 & 3,810 & 3,460 & 421 & 185 & 801 & 102 & 359 \\
\hline 1994 & 2,180 & 9,180 & 278 & 193 & 606 & 182 & 665 \\
\hline 1995 & 663 & 2,505 & 63.7 & 66.1 & 222 & 48.9 & 75.4 \\
\hline 1996 & 267 & 5,040 & 1,940 & 884 & 1,370 & 57.3 & 683 \\
\hline 1997 & 397 & 19,600 & 146 & 135 & 424 & 131 & 770 \\
\hline 1998 & 286 & 2,780 & -- & $5_{14.9}$ & 131 & 524.9 & 86.7 \\
\hline 1999 & 179 & 573 & -- & -- & -- & -- & -- \\
\hline
\end{tabular}

${ }^{1}$ Data from February to September

2 Data from April to September

${ }^{3}$ Data from December to September

${ }^{4}$ Data from July to September

5 Data from October to June 
Table 10. Total annual suspended-sediment loads per unit value of total streamflow at stream-gaging stations in the $\mathrm{H}-3 \mathrm{Highway}$ study area, Oahu, Hawaii, water years 1983-99

[All values are in tons per cubic feet per second-days (tons/(ft $3 / \mathrm{s}) \mathrm{d}$ ); only data for years with complete sediment records are listed; --, no data; station numbers are abbreviated, complete numbers are preceded by 16$]$

\begin{tabular}{|c|c|c|c|c|c|c|c|}
\hline \multirow{2}{*}{$\begin{array}{l}\text { Water } \\
\text { year }\end{array}$} & \multicolumn{7}{|c|}{ Station number } \\
\hline & 225800 & 226200 & 265600 & 270900 & 272200 & 273950 & 275000 \\
\hline 1983 & -- & -- & -- & -- & 0.04 & -- & -- \\
\hline 1984 & -- & 0.06 & 0.19 & -- & 0.05 & -- & -- \\
\hline 1985 & -- & 0.32 & 1.90 & 0.28 & 0.15 & -- & -- \\
\hline 1986 & -- & 0.23 & 1.16 & 0.61 & 0.11 & -- & -- \\
\hline 1987 & -- & 0.40 & 1.03 & 1.23 & 0.32 & -- & -- \\
\hline 1988 & -- & 0.95 & 1.76 & 0.95 & 0.37 & 0.18 & 1.03 \\
\hline 1989 & -- & 4.56 & 1.17 & 0.34 & 0.22 & 0.46 & 2.39 \\
\hline 1990 & -- & 1.23 & 2.18 & 0.11 & 0.14 & 0.09 & 1.87 \\
\hline 1991 & -- & 1.52 & 0.67 & 0.45 & 0.25 & 0.46 & 2.42 \\
\hline 1992 & 4.46 & 3.78 & 0.21 & 0.15 & 0.05 & 0.10 & 2.00 \\
\hline 1993 & 3.73 & 2.34 & 0.70 & 0.47 & 0.22 & 0.12 & 0.49 \\
\hline 1994 & 1.40 & 3.75 & 0.46 & 0.37 & 0.14 & 0.22 & 0.71 \\
\hline 1995 & 0.94 & 1.94 & 0.30 & 0.11 & 0.08 & 0.07 & 0.12 \\
\hline 1996 & 0.33 & 3.07 & 3.85 & 1.70 & 0.38 & 0.08 & 0.93 \\
\hline 1997 & 0.33 & 7.26 & 0.20 & 0.24 & 0.08 & 0.15 & 0.75 \\
\hline 1998 & 0.56 & 3.37 & -- & -- & 0.05 & -- & 0.12 \\
\hline 1999 & 0.32 & 0.47 & -- & -- & -- & -- & -- \\
\hline
\end{tabular}

Table 11. Total annual suspended-sediment yields at stream-gaging stations in the H-3 Highway study area, Oahu, Hawaii, water years 1983-99

[All values are in tons per square mile (tons $/ \mathrm{mi}^{2}$ ); only data for years with complete sediment records are listed; --, no data; station numbers are abbreviated, complete numbers are preceded by 16]

\begin{tabular}{|c|c|c|c|c|c|c|c|}
\hline \multirow{2}{*}{$\begin{array}{l}\text { Water } \\
\text { year }\end{array}$} & \multicolumn{7}{|c|}{ Station number } \\
\hline & 225800 & 226200 & 265600 & 270900 & 272200 & 273950 & 275000 \\
\hline 1983 & -- & -- & -- & -- & 45.4 & -- & -- \\
\hline 1984 & -- & 7.71 & 21.4 & -- & 20.1 & -- & -- \\
\hline 1985 & -- & 78.3 & 487 & 97.7 & 88.2 & -- & -- \\
\hline 1986 & -- & 90.3 & 425 & 207 & 84.5 & -- & -- \\
\hline 1987 & -- & 153 & 568 & 914 & 312 & -- & -- \\
\hline 1988 & -- & 873 & 1,560 & 1,100 & 588 & 468 & 1,350 \\
\hline 1989 & -- & 4,140 & 1,100 & 409 & 339 & 1,220 & 3,510 \\
\hline 1990 & -- & 564 & 1,040 & 120 & 156 & 184 & 1,710 \\
\hline 1991 & -- & 1,300 & 461 & 757 & 333 & 1,090 & 3,060 \\
\hline 1992 & 2,230 & 1,070 & 65 & 160 & 38.8 & 204 & 1,660 \\
\hline 1993 & 2,320 & 863 & 421 & 420 & 210 & 255 & 370 \\
\hline 1994 & 1,330 & 2,290 & 278 & 439 & 159 & 455 & 686 \\
\hline 1995 & 404 & 625 & 57.4 & 150 & 58.3 & 122 & 77.7 \\
\hline 1996 & 162 & 1,260 & 1,750 & 2,010 & 362 & 143 & 704 \\
\hline 1997 & 242 & 4,890 & 131 & 307 & 111 & 328 & 794 \\
\hline 1998 & 174 & 693 & -- & -- & 34.4 & -- & 89.4 \\
\hline 1999 & 109 & 143 & -- & -- & -- & -- & -- \\
\hline
\end{tabular}


Table 12. Statistical summary of selected water-quality data collected at station 16226200 , North Halawa Stream near Honolulu, Oahu, Hawaii, from May 1983 to March 1999

[inst., instantaneous; $\mu \mathrm{S} / \mathrm{cm}$ at $25^{\circ} \mathrm{C}$, microsiemens per centimeter at 25 degrees Celsius; $\mathrm{mg} / \mathrm{L}$, milligram per liter; $\mu \mathrm{g} / \mathrm{L}$, microgram per liter; <, less than; e, estimated using log-probability regression; $0.7 \mu \mathrm{m}-\mathrm{MF}, 0.7$ micron membrane filter; cols. $/ 100 \mathrm{~mL}$, colonies per 100 milliliters; $\mathrm{ft} 3 / \mathrm{s}$, cubic foot per second; ${ }^{\circ} \mathrm{C}$, degrees Celsius; $\mathrm{NTU}$, nephelometric turbidity unit; --, no data or not computed; Range of dates are the time periods of water-quality data collection during the specified construction activity]

\begin{tabular}{|c|c|c|c|c|c|c|c|c|}
\hline \multirow[b]{2}{*}{ Property or constituent } & \multirow{2}{*}{$\begin{array}{l}\text { Number } \\
\text { of } \\
\text { samples }\end{array}$} & \multirow{2}{*}{$\begin{array}{l}\text { Minimum } \\
\text { detection } \\
\text { limit }\end{array}$} & \multirow{2}{*}{$\begin{array}{l}\text { Number } \\
\text { of } \\
\text { detections }\end{array}$} & \multirow{2}{*}{$\begin{array}{l}\text { Mini- } \\
\text { mum }\end{array}$} & \multicolumn{3}{|c|}{ Concentration percentile } & \multirow{2}{*}{$\begin{array}{l}\text { Maxi- } \\
\text { mum }\end{array}$} \\
\hline & & & & & 25 & 50 & 75 & \\
\hline \multicolumn{9}{|c|}{ Before construction, May 1983 to August 1987, physical, biological, and organic } \\
\hline Discharge, inst., $\left(\mathrm{ft}^{3} / \mathrm{s}\right)$ & 23 & 0 & 23 & 0.03 & 0.20 & 1.8 & 3.9 & 37 \\
\hline \multicolumn{9}{|l|}{ Specific conductance } \\
\hline $\begin{array}{l}\left(\mu \mathrm{S} / \mathrm{cm} \text { at } 25^{\circ} \mathrm{C} \text { ) }\right. \\
\mathrm{pH} \text { (units) }\end{array}$ & $\begin{array}{l}23 \\
23\end{array}$ & $\begin{array}{l}0.1 \\
0.1\end{array}$ & $\begin{array}{l}23 \\
23\end{array}$ & $\begin{array}{r}69.0 \\
6.5\end{array}$ & $\begin{array}{r}120 \\
6.9\end{array}$ & $\begin{array}{r}135 \\
6.9\end{array}$ & $\begin{array}{r}145 \\
7.3\end{array}$ & $\begin{array}{l}180 \\
7.5\end{array}$ \\
\hline Temperature, water $\left({ }^{\circ} \mathrm{C}\right)$ & 23 & 0.5 & 23 & 19.0 & 21.0 & 21.8 & 23.0 & 24.5 \\
\hline Turbidity (NTU) & 11 & 0.1 & 11 & 0.4 & 1.2 & 1.5 & 6.8 & 14 \\
\hline Oxygen dissolved (mg/L) & 11 & 0.1 & 11 & 7.2 & 7.6 & 7.7 & 8.0 & 8.4 \\
\hline \multicolumn{8}{|l|}{ Fecal coliform $0.7 \mu \mathrm{m}-\mathrm{MF}$} & 6,200 \\
\hline \multicolumn{9}{|l|}{ Solids, residue at $105^{\circ} \mathrm{C}$} \\
\hline Carbon, organic, total $(\mathrm{mg} / \mathrm{L})$ & 5 & 0.1 & 5 & 0.6 & -- & 4.3 & -- & 7.1 \\
\hline Oil and grease, total (mg/L) & 5 & 1 & 1 & -- & -- & -- & -- & 5 \\
\hline \multicolumn{9}{|c|}{ During construction, November 1987 to November 1997, physical, biological, and organic } \\
\hline Discharge, inst., $\left(\mathrm{ft}^{3} / \mathrm{s}\right)$ & 90 & 0 & 90 & 0.01 & 0.07 & 0.5 & 2.9 & 229 \\
\hline \multicolumn{9}{|l|}{ Specific conductance } \\
\hline $\mathrm{pH}$ (units) & 90 & 0.1 & 90 & 6.6 & 7.5 & 7.7 & 8.0 & 8.3 \\
\hline Temperature, water $\left({ }^{\circ} \mathrm{C}\right)$ & 90 & 0.5 & 90 & 17.5 & 21.5 & 22.5 & 24.0 & 26.5 \\
\hline Turbidity (NTU) & 88 & 0.1 & 88 & 0.2 & 0.8 & 2.1 & 32 & 2,000 \\
\hline Oxygen dissolved (mg/L) & 90 & 0.1 & 90 & 5.6 & 7.4 & 8.1 & 8.5 & 9.4 \\
\hline \multicolumn{7}{|l|}{ Fecal coliform $0.7 \mu \mathrm{m}-\mathrm{MF}$} & 1,300 & 16,000 \\
\hline \multicolumn{6}{|l|}{ Solids, residue at $105^{\circ} \mathrm{C}$} & $\mathrm{e} 5.5$ & e31 & 680 \\
\hline Carbon, organic, total (mg/L) & 18 & 0.1 & 18 & 0.8 & 1.2 & 2.4 & 5.2 & 89 \\
\hline Oil and grease, total (mg/L) & 18 & 1 & 0 & -- & -- & -- & -- & -- \\
\hline \multicolumn{9}{|c|}{ After construction, December 1997 to March 1999, physical, biological, and organic } \\
\hline Discharge, inst., $\left(\mathrm{ft}^{3} / \mathrm{s}\right)$ & 11 & 0 & 11 & 0.01 & 0.02 & 0.16 & 35 & 44 \\
\hline \multicolumn{9}{|l|}{ Specific conductance } \\
\hline$\left(\mu \mathrm{S} / \mathrm{cm}\right.$ at $\left.25^{\circ} \mathrm{C}\right)$ & 8 & 0.1 & 8 & 144 & 192 & 336 & 357 & 386 \\
\hline $\mathrm{pH}$ (units) & 8 & 0.1 & 8 & 7.3 & 7.5 & 7.6 & 7.8 & 7.9 \\
\hline Temperature, water $\left({ }^{\circ} \mathrm{C}\right)$ & 8 & 0.5 & 8 & 18.0 & 20.0 & 22.0 & 23.0 & 23.5 \\
\hline Turbidity (NTU) & 11 & 0.1 & 11 & 0.2 & 0.3 & 2.5 & 20 & 390 \\
\hline Oxygen dissolved (mg/L) & 8 & 0.1 & 8 & 6.6 & 7.4 & 7.8 & 8.6 & 8.7 \\
\hline $\begin{array}{l}\text { Fecal coliform } 0.7 \mu \mathrm{m}-\mathrm{MF} \\
(\text { cols. } / 100 \mathrm{~mL})\end{array}$ & 2 & 0 & 2 & 130 & -- & -- & -- & 1,600 \\
\hline $\begin{array}{l}\text { Solids, residue at } 105^{\circ} \mathrm{C}, \\
\text { suspended }(\mathrm{mg} / \mathrm{L})\end{array}$ & 8 & 1 & 8 & 1 & 4 & 40 & 73 & 1,030 \\
\hline Carbon, organic, total (mg/L) & 4 & 0.1 & 4 & 1.4 & -- & -- & -- & 10 \\
\hline Oil and grease, total (mg/L) & 4 & 1 & 0 & -- & -- & -- & -- & -- \\
\hline
\end{tabular}


Table 12. Statistical summary of selected water-quality data collected at station 16226200, North Halawa Stream near Honolulu, Oahu, Hawaii, from May 1983 to March 1999--Continued

\begin{tabular}{|c|c|c|c|c|c|c|c|c|}
\hline Property or constituent & $\begin{array}{c}\text { Number } \\
\text { of } \\
\text { samples }\end{array}$ & $\begin{array}{l}\text { Minimum } \\
\text { detection } \\
\text { limit }\end{array}$ & $\begin{array}{c}\text { Number } \\
\text { of } \\
\text { detections }\end{array}$ & $\begin{array}{l}\text { Mini- } \\
\text { mum }\end{array}$ & \multicolumn{3}{|c|}{ Concentration percentile } & $\begin{array}{l}\text { Maxi- } \\
\text { mum }\end{array}$ \\
\hline \multicolumn{9}{|c|}{ Before construction, May 1983 to August 1987 , major ions and nutrients $(\mathrm{mg} / \mathrm{L})$} \\
\hline Calcium, dissolved & 5 & 0.02 & 5 & 2.9 & -- & 4.9 & -- & 5.7 \\
\hline Magnesium, dissolved & 5 & 0.01 & 5 & 2.3 & -- & 4.4 & -- & 5.5 \\
\hline Sodium, dissolved & 5 & 0.2 & 5 & 7.4 & -- & 12 & -- & 13 \\
\hline Potassium, dissolved & 5 & 0.1 & 5 & 0.7 & -- & 0.8 & -- & 1.0 \\
\hline Alkalinity $\left(\right.$ as $\left.\mathrm{CaCO}_{3}\right)$ & 5 & 1 & 5 & 19 & -- & 29 & -- & 40 \\
\hline Sulfate, dissolved & 5 & 0.1 & 5 & 3.5 & -- & 4.9 & -- & 7.4 \\
\hline Chloride, dissolved & 5 & 0.1 & 5 & 11 & -- & 17 & -- & 18 \\
\hline Fluoride, dissolved & 5 & 0.1 & 0 & -- & -- & -- & -- & -- \\
\hline Silica, dissolved & 5 & 0.01 & 5 & 8.8 & -- & 20 & -- & 21 \\
\hline $\begin{array}{l}\text { Nitrogen, nitrite plus } \\
\text { nitrate, total (as N) }\end{array}$ & 7 & 0.05 & 0 & -- & -- & -- & -- & -- \\
\hline $\begin{array}{l}\text { Nitrogen, ammonia } \\
\text { plus organic, total (as N) }\end{array}$ & 8 & 0.2 & 6 & $<0.2$ & $\mathrm{e} 0.22$ & $\mathrm{e} 0.40$ & $\mathrm{e} 0.78$ & 0.80 \\
\hline Phosphorus, total (as P) & 8 & 0.01 & 6 & $<0.01$ & $\mathrm{e} 0.01$ & $\mathrm{e} 0.02$ & $\mathrm{e} 0.04$ & 0.06 \\
\hline \multicolumn{9}{|c|}{ During construction, November 1987 to November 1997, major ions and nutrients (mg/L) } \\
\hline Hardness, total $\left(\right.$ as $\left.\mathrm{CaCO}_{3}\right)$ & ) 18 & 1 & 18 & 12 & 44 & 57 & 90 & 140 \\
\hline Calcium, dissolved & 18 & 0.02 & 18 & 2 & 7.6 & 12 & 16 & 28 \\
\hline Magnesium, dissolved & 18 & 0.01 & 18 & 1.7 & 5.6 & 6.9 & 11 & 16 \\
\hline Sodium, dissolved & 18 & 0.2 & 18 & 6.2 & 13 & 16 & 20 & 23 \\
\hline Potassium, dissolved & 18 & 0.1 & 18 & 0.9 & 0.9 & 1.2 & 1.3 & 1.5 \\
\hline Alkalinity $\left(\right.$ as $\left.\mathrm{CaCO}_{3}\right)$ & 18 & 1 & 18 & 11 & 44 & 60 & 86 & 124 \\
\hline Sulfate, dissolved & 18 & 0.1 & 18 & 3.8 & 5.0 & 6.9 & 8.0 & 11 \\
\hline Chloride, dissolved & 18 & 0.1 & 18 & 9.8 & 18 & 19 & 31 & 43 \\
\hline Fluoride, dissolved & 18 & 0.1 & 6 & $<0.1$ & -- & -- & -- & 0.1 \\
\hline Silica, dissolved & 18 & 0.01 & 18 & 6.9 & 16 & 20 & 23 & 25 \\
\hline $\begin{array}{l}\text { Nitrogen, nitrite plus } \\
\text { nitrate, total (as N) }\end{array}$ & 19 & 0.05 & 7 & $<0.1$ & $\mathrm{e} 0.01$ & $\mathrm{e} 0.02$ & $\mathrm{e} 0.1$ & 1.0 \\
\hline $\begin{array}{l}\text { Nitrogen, nitrite plus } \\
\text { nitrate, dissolved (as N) }\end{array}$ & 13 & 0.05 & 8 & $<0.05$ & $\mathrm{e} 0.03$ & $\mathrm{e} 0.06$ & $\mathrm{e} 0.10$ & 0.18 \\
\hline $\begin{array}{l}\text { Nitrogen, ammonia } \\
\text { plus organic, total (as N) }\end{array}$ & 36 & 0.2 & 9 & $<0.2$ & -- & $\mathrm{e} 0.03$ & $\mathrm{e} 0.18$ & 2.8 \\
\hline Phosphorus, total (as P) & 36 & 0.01 & 21 & $<0.01$ & -- & $\mathrm{e} 0.02$ & $\mathrm{e} 0.04$ & 0.28 \\
\hline \multicolumn{9}{|c|}{ After construction, December 1997 to March 1999, major ions and nutrients (mg/L) } \\
\hline Hardness, total (as $\left.\mathrm{CaCO}_{3}\right)$ & ) 1 & 1 & 1 & -- & -- & -- & -- & 130 \\
\hline Calcium, dissolved & 1 & 0.02 & 1 & -- & -- & -- & -- & 27 \\
\hline Magnesium, dissolved & 1 & 0.01 & 1 & -- & -- & -- & -- & 16 \\
\hline Sodium, dissolved & 1 & 0.2 & 1 & -- & -- & -- & -- & 27 \\
\hline Potassium, dissolved & 1 & 0.1 & 1 & -- & -- & -- & -- & 1.5 \\
\hline Alkalinity $\left(\right.$ as $\mathrm{CaCO}_{3}$ ) & 1 & 1 & 1 & -- & -- & -- & -- & 137 \\
\hline Sulfate, dissolved & 4 & 0.1 & 4 & 3.9 & -- & -- & -- & 12 \\
\hline Chloride, dissolved & 4 & 0.1 & 4 & 15 & -- & -- & -- & 40 \\
\hline Fluoride, dissolved & 1 & 0.1 & 0 & -- & -- & -- & -- & -- \\
\hline Silica, dissolved & 1 & 0.01 & 1 & -- & -- & -- & -- & 21 \\
\hline $\begin{array}{l}\text { Nitrogen, nitrite plus } \\
\text { nitrate, dissolved (as N) }\end{array}$ & 5 & 0.05 & 2 & -- & -- & -- & -- & 0.07 \\
\hline $\begin{array}{l}\text { Nitrogen, ammonia } \\
\text { plus organic, total (as N) }\end{array}$ & 5 & 0.1 & 4 & -- & -- & -- & -- & 0.86 \\
\hline Phosphorus, total (as P) & 5 & 0.05 & 1 & -- & -- & -- & -- & 0.11 \\
\hline
\end{tabular}


Table 12. Statistical summary of selected water-quality data collected at station 16226200, North Halawa Stream near Honolulu, Oahu Hawaii, from May 1983 to March 1999--Continued

\begin{tabular}{|c|c|c|c|c|c|c|c|c|}
\hline \multirow[b]{2}{*}{ Property or constituent } & \multirow{2}{*}{$\begin{array}{l}\text { Number } \\
\text { of } \\
\text { samples }\end{array}$} & \multirow{2}{*}{$\begin{array}{l}\text { Minimum } \\
\text { detection } \\
\text { limit }\end{array}$} & \multirow{2}{*}{$\begin{array}{l}\text { Number } \\
\text { of } \\
\text { detections }\end{array}$} & \multirow{2}{*}{$\begin{array}{l}\text { Mini- } \\
\text { mum }\end{array}$} & \multicolumn{3}{|c|}{$\underline{\text { Concentration percentile }}$} & \multirow{2}{*}{$\begin{array}{l}\text { Maxi- } \\
\text { mum }\end{array}$} \\
\hline & & & & & 25 & 50 & 75 & \\
\hline \multicolumn{9}{|c|}{ Before construction, May 1983 to August 1987, trace metals $(\mu \mathrm{g} / \mathrm{L})$} \\
\hline Aluminum, total recoverable & le 5 & 10 & 5 & 50 & -- & 80 & -- & 540 \\
\hline Aluminum, dissolved & 5 & 10 & 5 & 30 & -- & 60 & -- & 240 \\
\hline Cadmium, total recoverable & 5 & 1 & 0 & -- & -- & -- & -- & -- \\
\hline Cadmium, dissolved & 5 & 1 & 0 & -- & -- & -- & -- & -- \\
\hline Chromium, total recoverable & 5 & 10 & 1 & -- & -- & -- & -- & 10 \\
\hline Chromium, dissolved & 5 & 1 & 1 & -- & -- & -- & 1 & \\
\hline Copper, total recoverable & 5 & 1 & 5 & 2 & -- & 5 & -- & 6 \\
\hline Copper, dissolved & 5 & 1 & 4 & $<1$ & -- & 1 & -- & 6 \\
\hline Iron, total recoverable & 5 & 10 & 5 & 20 & -- & 70 & -- & 1,300 \\
\hline Iron, dissolved & 5 & 3 & 5 & 24 & -- & 44 & -- & 160 \\
\hline Lead, total recoverable & 5 & 1 & 2 & -- & -- & -- & -- & 11 \\
\hline Lead, dissolved & 5 & 1 & 2 & -- & -- & -- & -- & 5 \\
\hline Nickel, total recoverable & 5 & 1 & 4 & $<1$ & -- & 4 & -- & 13 \\
\hline Nickel, dissolved & 5 & 1 & 2 & -- & -- & -- & -- & 1 \\
\hline Zinc, total recoverable & 5 & 10 & 4 & $<10$ & -- & 30 & -- & 270 \\
\hline Zinc, dissolved & 5 & 3 & 5 & 4 & -- & 5.0 & -- & 20 \\
\hline \multicolumn{9}{|c|}{ During construction, November 1987 to November 1997 , trace metals $(\mu \mathrm{g} / \mathrm{L})$} \\
\hline Aluminum, total recoverable & le 18 & 10 & 18 & 40 & 95 & 240 & 1,800 & 180,000 \\
\hline Aluminum, dissolved & 18 & 10 & 14 & $<10$ & e9 & e30 & e93 & 340 \\
\hline Cadmium, total recoverable & 18 & 1 & 0 & -- & -- & -- & -- & -- \\
\hline Cadmium, dissolved & 6 & 1 & 0 & -- & -- & -- & -- & -- \\
\hline Chromium, total recoverable & 18 & 1 & 11 & $<1$ & e.09 & $\mathrm{e} 1$ & $\mathrm{e} 7$ & 190 \\
\hline Chromium, dissolved & 6 & 1 & 0 & -- & -- & -- & -- & -- \\
\hline Copper, total recoverable & 18 & 1 & 15 & $<1$ & $\mathrm{e} 1$ & e1 & $\mathrm{e} 5.2$ & 59 \\
\hline Copper, dissolved & 6 & 1 & 0 & -- & -- & -- & -- & -- \\
\hline Iron, total recoverable & 18 & 10 & 18 & 90 & 190 & 400 & 2,100 & 51,000 \\
\hline Iron, dissolved & 18 & 3 & 17 & $<3$ & e21 & $\mathrm{e} 50$ & e90 & 230 \\
\hline Lead, total recoverable & 18 & 1 & 5 & $<1$ & $\mathrm{e} 0.3$ & $\mathrm{e} 0.5$ & e1 & 2 \\
\hline Lead, dissolved & 6 & 1 & 0 & -- & -- & -- & -- & -- \\
\hline Nickel, total recoverable & 18 & 1 & 13 & $<1$ & $\mathrm{e} 0.3$ & $\mathrm{e} 1.5$ & $\mathrm{e} 5.2$ & 92 \\
\hline Nickel, dissolved & 18 & 1 & 5 & $<1$ & $\mathrm{e} 0.3$ & $\mathrm{e} 0.5$ & e1 & 2 \\
\hline Zinc, total recoverable & 18 & 10 & 9 & $<10$ & e5 & e15 & $\mathrm{e} 22$ & 100 \\
\hline Zinc, dissolved & 6 & 3 & 5 & $<3$ & $\mathrm{e} 4$ & e5 & e8 & 9 \\
\hline \multicolumn{9}{|c|}{ After construction, December 1997 to March 1999, trace metals $(\mu \mathrm{g} / \mathrm{L})$} \\
\hline Aluminum, total recoverable & le 1 & 10 & 1 & -- & -- & -- & -- & 20 \\
\hline Aluminum, dissolved & 1 & 10 & 0 & -- & -- & -- & -- & -- \\
\hline Cadmium, total recoverable & 1 & 1 & 0 & -- & -- & -- & -- & -- \\
\hline Cadmium, dissolved & 0 & 1 & 0 & -- & -- & -- & -- & -- \\
\hline Chromium, total recoverable & 2 & 1 & 0 & -- & -- & -- & -- & -- \\
\hline Chromium, dissolved & 3 & 1 & 0 & -- & -- & -- & -- & -- \\
\hline Copper, total recoverable & 2 & 1 & 0 & -- & -- & -- & -- & -- \\
\hline Copper, dissolved & 3 & 1 & 0 & -- & -- & -- & -- & -- \\
\hline Iron, total recoverable & 1 & 10 & 1 & -- & -- & -- & -- & 80 \\
\hline Iron, dissolved & 1 & 3 & 1 & -- & -- & -- & -- & 31 \\
\hline Lead, total recoverable & 2 & 1 & 0 & -- & -- & -- & -- & -- \\
\hline Lead, dissolved & 3 & 1 & 0 & -- & -- & -- & -- & -- \\
\hline Nickel, total recoverable & 2 & 1 & 0 & -- & -- & -- & -- & -- \\
\hline Nickel, dissolved & 4 & 1 & 0 & -- & -- & -- & -- & -- \\
\hline Zinc, total recoverable & 2 & 10 & 0 & -- & -- & -- & -- & -- \\
\hline Zinc, dissolved & 3 & 20 & 0 & -- & -- & -- & -- & -- \\
\hline
\end{tabular}


Table 13. Statistical summary of selected water-quality data collected at station 16227100 , Halawa Stream below H-1, Oahu, Hawaii, from November 1988 to November 1998

[inst., instantaneous; $\mu \mathrm{S} / \mathrm{cm}$ at $25^{\circ} \mathrm{C}$, microsiemens per centimeter at 25 degrees Celsius; $\mathrm{mg} / \mathrm{L}$, milligram per liter; $\mu \mathrm{g} / \mathrm{L}$, microgram per liter; <, less than; e, estimated using log-probability regression; $0.7 \mu \mathrm{m}-\mathrm{MF}, 0.7$ micron membrane filter; cols. $/ 100 \mathrm{~mL}$, colonies per 100 milliliters; $\mathrm{ft}^{3} / \mathrm{s}$, cubic foot per second; ${ }^{\circ} \mathrm{C}$, degrees Celsius; $\mathrm{NTU}$, nephelometric turbidity unit; --, no data or not computed; Range of dates are the time periods of water-quality data collection during the specified construction activity]

\begin{tabular}{|c|c|c|c|c|c|c|c|c|}
\hline Property or constituent & $\begin{array}{l}\text { Number } \\
\text { of } \\
\text { samples }\end{array}$ & $\begin{array}{l}\text { Minimum } \\
\text { detection } \\
\text { limit }\end{array}$ & $\begin{array}{l}\text { Number } \\
\text { of } \\
\text { detections }\end{array}$ & $\begin{array}{l}\text { Mini- } \\
\text { mum }\end{array}$ & $\frac{\text { Conce }}{25}$ & $\frac{\text { entration pe }}{50}$ & $\frac{\text { ercentile }}{75}$ & $\begin{array}{l}\text { Maxi- } \\
\text { mum }\end{array}$ \\
\hline \multicolumn{9}{|c|}{ During construction, November 1988 to November 1997, physical, biological, and organic } \\
\hline Discharge, inst., $\left(\mathrm{ft}^{3} / \mathrm{s}\right)$ & 88 & 0 & 87 & 0 & 0.22 & 0.68 & 3.0 & 750 \\
\hline \multicolumn{9}{|l|}{ Specific conductance } \\
\hline$\left(\mu \mathrm{S} / \mathrm{cm}\right.$ at $\left.25^{\circ} \mathrm{C}\right)$ & 89 & 1 & 89 & 90 & 328 & 570 & 770 & 1,500 \\
\hline $\mathrm{pH}$ (units) & 89 & 0.1 & 89 & 6.7 & 8.6 & 9.0 & 9.3 & 10.1 \\
\hline Temperature, water $\left({ }^{\circ} \mathrm{C}\right)$ & 89 & 0.5 & 89 & 19.5 & 24.0 & 27.5 & 29.8 & 37.0 \\
\hline Turbidity (NTU) & 87 & 0.1 & 87 & 0.3 & 1.0 & 2.0 & 4.2 & 400 \\
\hline Oxygen dissolved (mg/L) & 89 & 0.1 & 89 & 6.0 & 9.6 & 10.7 & 11.4 & 14.0 \\
\hline $\begin{array}{l}\text { Fecal coliform } 0.7 \mu \mathrm{m}-\mathrm{MF} \\
(\text { cols. } / 100 \mathrm{~mL})\end{array}$ & 33 & 0 & 33 & 97 & 930 & 2,000 & 5,450 & 50,000 \\
\hline $\begin{array}{l}\text { Solids, residue at } 105^{\circ} \mathrm{C}, \\
\text { suspended }(\mathrm{mg} / \mathrm{L})\end{array}$ & 33 & 1 & 28 & 4 & $\mathrm{e} 4.5$ & e9.0 & e16.5 & 405 \\
\hline Carbon, organic, total $(\mathrm{mg} / \mathrm{L})$ & L) 17 & 0.1 & 17 & 1.4 & 3.0 & 4.6 & 6.4 & 52.0 \\
\hline Oil and grease, total $(\mathrm{mg} / \mathrm{L})$ & 16 & 1 & 1 & -- & -- & -- & -- & 1 \\
\hline \multicolumn{9}{|c|}{ After construction, December 1997 to November 1998, physical, biological, and organic } \\
\hline Discharge, inst., $\left(\mathrm{ft}^{3} / \mathrm{s}\right)$ & 4 & 0 & 4 & 0.01 & -- & 2.4 & -- & 4.8 \\
\hline \multicolumn{9}{|l|}{ Specific conductance } \\
\hline$\left(\mu \mathrm{S} / \mathrm{cm}\right.$ at $\left.25^{\circ} \mathrm{C}\right)$ & 4 & 1 & 4 & 173 & -- & 542 & -- & 912 \\
\hline $\mathrm{pH}$ (units) & 4 & 0.1 & 4 & 8.7 & -- & 8.9 & -- & 9.1 \\
\hline Temperature, water $\left({ }^{\circ} \mathrm{C}\right)$ & 4 & 0.5 & 4 & 22.5 & -- & 25.0 & -- & 27.5 \\
\hline Turbidity (NTU) & 4 & 0.1 & 4 & 0.66 & -- & 1.3 & -- & 2.0 \\
\hline Oxygen dissolved (mg/L) & 4 & 0.1 & 4 & 9.7 & -- & 10.8 & -- & 11.9 \\
\hline $\begin{array}{l}\text { Fecal coliform } 0.7 \mu \mathrm{m}-\mathrm{MF} \\
\quad(\text { cols. } / 100 \mathrm{~mL})\end{array}$ & 2 & 0 & 2 & 1,800 & -- & -- & -- & 2,800 \\
\hline $\begin{array}{l}\text { Solids, residue at } 105^{\circ} \mathrm{C}, \\
\text { suspended }(\mathrm{mg} / \mathrm{L})\end{array}$ & 3 & 1 & 3 & 4.0 & -- & 6.0 & -- & 8.0 \\
\hline Carbon, organic, total $(\mathrm{mg} / \mathrm{L})$ & 1 & 0.1 & 1 & -- & -- & -- & -- & 2.9 \\
\hline Oil and grease, total $(\mathrm{mg} / \mathrm{L})$ & 1 & 1 & 0 & -- & -- & -- & -- & -- \\
\hline
\end{tabular}


Table 13. Statistical summary of selected water-quality data collected at station 16227100, Halawa Stream below H-1, Oahu, Hawaii, from November 1988 to November 1998--Continued

\begin{tabular}{|c|c|c|c|c|c|c|c|c|}
\hline \multirow[b]{2}{*}{ Property or constituent } & \multirow{2}{*}{$\begin{array}{l}\text { Number } \\
\text { of } \\
\text { samples }\end{array}$} & \multirow{2}{*}{$\begin{array}{l}\text { Minimum } \\
\text { detection } \\
\text { limit }\end{array}$} & \multirow{2}{*}{$\begin{array}{l}\text { Number } \\
\text { of } \\
\text { detections }\end{array}$} & \multirow{2}{*}{$\begin{array}{l}\text { Mini- } \\
\text { mum }\end{array}$} & \multicolumn{3}{|c|}{ Concentration percentile } & \multirow{2}{*}{$\begin{array}{l}\text { Maxi- } \\
\text { mum }\end{array}$} \\
\hline & & & & & 25 & 50 & 75 & \\
\hline \multicolumn{9}{|c|}{ During construction, November 1988 to December 1997, major ions and nutrients (mg/L) } \\
\hline Hardness, total (as $\left.\mathrm{CaCO}_{3}\right)$ & ) 17 & 1 & 17 & 30 & 72 & 130 & 235 & 270 \\
\hline Calcium, dissolved & 17 & 0.02 & 17 & 6.7 & 15 & 25 & 38 & 50 \\
\hline Magnesium, dissolved & 17 & 0.01 & 17 & 3.3 & 8.8 & 15 & 34 & 36 \\
\hline Sodium, dissolved & 17 & 0.2 & 17 & 12 & 24 & 43 & 72 & 97 \\
\hline Potassium, dissolved & 17 & 0.1 & 17 & 1.1 & 1.5 & 1.8 & 3.7 & 5.1 \\
\hline Alkalinity $\left(\right.$ as $\left.\mathrm{CaCO}_{3}\right)$ & 16 & 1 & 16 & 30 & 48 & 72 & 100 & 163 \\
\hline Sulfate, dissolved & 17 & 0.1 & 17 & 6 & 10 & 21 & 36 & 53 \\
\hline Chloride, dissolved & 17 & 0.1 & 17 & 14 & 35 & 71 & 160 & 310 \\
\hline Fluoride, dissolved & 17 & 0.1 & 10 & 0.1 & $\mathrm{e} 0.1$ & $\mathrm{e} 0.1$ & $\mathrm{e} 0.1$ & 0.2 \\
\hline Silica, dissolved & 17 & 0.01 & 17 & 11 & 15 & 21 & 24 & 34 \\
\hline $\begin{array}{l}\text { Nitrogen, nitrite plus } \\
\text { nitrate, total (as N) }\end{array}$ & 18 & 0.05 & 8 & $<0.1$ & $\mathrm{e} 0.01$ & $\mathrm{e} 0.04$ & $\mathrm{e} 0.24$ & 2.7 \\
\hline $\begin{array}{l}\text { Nitrogen, nitrite plus } \\
\text { nitrate, dissolved (as N) }\end{array}$ & 12 & 0.05 & 4 & $<0.05$ & -- & -- & -- & 0.29 \\
\hline $\begin{array}{l}\text { Nitrogen, ammonia } \\
\text { plus organic, total (as N) }\end{array}$ & 34 & 0.2 & 26 & $<0.2$ & e0.19 & $\mathrm{e} 0.30$ & $\mathrm{e} 0.50$ & 1.5 \\
\hline Phosphorus, total (as P) & 34 & 0.01 & 26 & $<0.01$ & $\mathrm{e} 0.01$ & $\mathrm{e} 0.03$ & $\mathrm{e} 0.05$ & 0.15 \\
\hline \multicolumn{9}{|c|}{ After construction, December 1997 to November 1998, major ions and nutrients (mg/L) } \\
\hline Hardness, total (as $\left.\mathrm{CaCO}_{3}\right)$ & ) 0 & 1 & 0 & -- & -- & -- & -- & -- \\
\hline Calcium, dissolved & 0 & 0.02 & 0 & -- & -- & -- & -- & -- \\
\hline Magnesium, dissolved & 0 & 0.01 & 0 & -- & -- & -- & -- & -- \\
\hline Sodium, dissolved & 0 & 0.2 & 0 & -- & -- & -- & -- & -- \\
\hline Potassium, dissolved & 0 & 0.1 & 0 & -- & -- & -- & -- & -- \\
\hline Alkalinity $\left(\right.$ as $\mathrm{CaCO}_{3}$ ) & 0 & 1 & 0 & -- & -- & -- & -- & -- \\
\hline Sulfate, dissolved & 1 & 0.1 & 1 & -- & -- & -- & -- & 5.8 \\
\hline Chloride, dissolved & 1 & 0.1 & 1 & -- & -- & -- & -- & 17 \\
\hline Fluoride, dissolved & 0 & 0.1 & 0 & -- & -- & -- & -- & -- \\
\hline Silica, dissolved & 0 & 0.01 & 0 & -- & -- & -- & -- & -- \\
\hline $\begin{array}{l}\text { Nitrogen, nitrite plus } \\
\text { nitrate, dissolved (as N) }\end{array}$ & 3 & 0.05 & 0 & -- & -- & -- & -- & -- \\
\hline $\begin{array}{l}\text { Nitrogen, ammonia } \\
\text { plus organic, total (as } \mathrm{N} \text { ) }\end{array}$ & 3 & 0.1 & 3 & 0.15 & -- & 0.35 & -- & 0.55 \\
\hline Phosphorus, total (as P) & 3 & 0.05 & 1 & -- & -- & -- & -- & 0.16 \\
\hline
\end{tabular}


Table 13. Statistical summary of selected water-quality data collected at station 16227100 , Halawa Stream below H-1, Oahu, Hawaii, from November 1988 to November 1998--Continued

\begin{tabular}{|c|c|c|c|c|c|c|c|c|}
\hline Property or constituent & $\begin{array}{l}\text { Number } \\
\text { of } \\
\text { samples }\end{array}$ & $\begin{array}{l}\text { Minimum } \\
\text { detection } \\
\text { limit }\end{array}$ & $\begin{array}{l}\text { Number } \\
\text { of } \\
\text { detections }\end{array}$ & $\begin{array}{l}\text { Mini- } \\
\text { mum }\end{array}$ & $\frac{\text { Conce }}{25}$ & $\frac{\text { tration pe }}{50}$ & $\frac{\text { centile }}{75}$ & $\begin{array}{l}\text { Maxi- } \\
\text { mum }\end{array}$ \\
\hline \multicolumn{9}{|c|}{ During construction, November 1988 to November 1997, trace metals $(\mu \mathrm{g} / \mathrm{L})$} \\
\hline Aluminum, total recoverable & e 17 & 10 & 17 & 40 & 120 & 230 & 1300 & 550,000 \\
\hline Aluminum, dissolved & 17 & 10 & 9 & 10 & $\mathrm{e} 2.0$ & e10 & e35 & 300 \\
\hline Cadmium, total recoverable & 17 & 1 & 2 & $<1$ & -- & -- & -- & 2 \\
\hline Cadmium, dissolved & 6 & 1 & 0 & -- & -- & -- & -- & -- \\
\hline Chromium, total recoverable & e 17 & 1 & 11 & $<1$ & $\mathrm{e} 0.21$ & $\mathrm{e} 2.0$ & $\mathrm{e} 7.5$ & 230 \\
\hline Chromium, dissolved & 6 & 1 & 3 & $<1$ & -- & -- & -- & 9 \\
\hline Copper, total recoverable & 17 & 1 & 17 & 2 & 3 & 4 & 8 & 130 \\
\hline Copper, dissolved & 6 & 1 & 6 & 2 & 3 & 4 & 6 & 8 \\
\hline Iron, total recoverable & 17 & 10 & 17 & 90 & 175 & 320 & 1400 & 76,000 \\
\hline Iron, dissolved & 17 & 3 & 17 & 5 & 10 & 13 & 48 & 580 \\
\hline Lead, total recoverable & 17 & 1 & 8 & 1 & $\mathrm{e} 0.10$ & $\mathrm{e} 0.54$ & $\mathrm{e} 3.5$ & 70 \\
\hline Lead, dissolved & 6 & 1 & 4 & $<1$ & -- & -- & -- & 1 \\
\hline Nickel, total recoverable & 17 & 1 & 16 & $<1$ & $\mathrm{e} 1.5$ & $\mathrm{e} 2.0$ & $\mathrm{e} 3.5$ & 140 \\
\hline Nickel, dissolved & 17 & 1 & 7 & $<1$ & $\mathrm{e} 0.45$ & $\mathrm{e} 0.67$ & $\mathrm{e} 1.0$ & 2.0 \\
\hline Zinc, total recoverable & 17 & 10 & 9 & $<10$ & $\mathrm{e} 2.8$ & e10 & e35 & 200 \\
\hline Zinc, dissolved & 6 & 3 & 5 & $<3$ & e3.6 & e11 & e22 & 22 \\
\hline \multicolumn{9}{|c|}{ After construction, December 1997 to November 1998, trace metals $(\mu \mathrm{g} / \mathrm{L})$} \\
\hline Aluminum, total recoverable & e 0 & 10 & 0 & -- & - & -- & -- & \\
\hline Aluminum, dissolved & 0 & 10 & 0 & -- & -- & -- & -- & -- \\
\hline Cadmium, total recoverable & 0 & 1 & 0 & -- & -- & -- & -- & -- \\
\hline Cadmium, dissolved & 0 & 1 & 0 & -- & -- & -- & -- & -- \\
\hline Chromium, total recoverable & 0 & 1 & 0 & -- & -- & -- & -- & -- \\
\hline Chromium, dissolved & 0 & 1 & 0 & -- & -- & -- & -- & -- \\
\hline Copper, total recoverable & 0 & 1 & 0 & -- & -- & -- & -- & -- \\
\hline Copper, dissolved & 0 & 1 & 0 & -- & -- & -- & -- & -- \\
\hline Iron, total recoverable & 0 & 10 & 0 & -- & -- & -- & -- & \\
\hline Iron, dissolved & 0 & 3 & 0 & -- & -- & -- & -- & \\
\hline Lead, total recoverable & 0 & 1 & 0 & -- & -- & -- & -- & -- \\
\hline Lead, dissolved & 0 & 1 & 0 & -- & -- & -- & -- & -- \\
\hline Nickel, total recoverable & 0 & 1 & 0 & -- & -- & -- & -- & -- \\
\hline Nickel, dissolved & 0 & 1 & 0 & -- & -- & -- & -- & -- \\
\hline Zinc, total recoverable & 0 & 10 & 0 & -- & -- & -- & -- & -- \\
\hline Zinc, dissolved & 0 & 20 & 0 & -- & -- & -- & -- & -- \\
\hline
\end{tabular}


Table 14. Statistical summary of selected water-quality data collected at station 16265600, Right Branch Kamooalii Stream near Kaneohe, Oahu, Hawaii, from February 1983 to January 1998

[inst., instantaneous; $\mu \mathrm{S} / \mathrm{cm}$ at $25^{\circ} \mathrm{C}$, microsiemens per centimeter at 25 degrees Celsius; $\mathrm{mg} / \mathrm{L}$, milligram per liter; $\mu \mathrm{g} / \mathrm{L}$, microgram per liter; <, less than; e, estimated using log-probability regression; $0.7 \mu \mathrm{m}-\mathrm{MF}, 0.7$ micron membrane filter; cols./100 mL, colonies per 100 milliliters; $\mathrm{ft}^{3} / \mathrm{s}$, cubic foot per second; ${ }^{\circ} \mathrm{C}$, degrees Celsius; $\mathrm{NTU}$, nephelometric turbidity unit; --, no data or not computed; Range of dates are the time periods of water-quality data collection during the specified construction activity]

\begin{tabular}{|c|c|c|c|c|c|c|c|c|}
\hline Property or constituent & $\begin{array}{l}\text { Number } \\
\text { of } \\
\text { samples }\end{array}$ & $\begin{array}{l}\text { Minimum } \\
\text { detection } \\
\text { limit }\end{array}$ & $\begin{array}{l}\text { Number } \\
\text { of } \\
\text { detections }\end{array}$ & $\begin{array}{l}\text { Mini- } \\
\text { mum }\end{array}$ & \multicolumn{3}{|c|}{ Concentration percentile } & $\begin{array}{l}\text { Maxi- } \\
\text { mum }\end{array}$ \\
\hline \multicolumn{9}{|c|}{ Before construction, February 1983, physical, biological, and organic } \\
\hline Discharge, inst., $\left(\mathrm{ft}^{3} / \mathrm{s}\right)$ & 1 & 0 & 1 & -- & -- & -- & -- & 1.8 \\
\hline Specific conductance & & & & & & & & \\
\hline$\left(\mu \mathrm{S} / \mathrm{cm}\right.$ at $\left.25^{\circ} \mathrm{C}\right)$ & 1 & 0.1 & 1 & -- & -- & -- & -- & 196 \\
\hline $\mathrm{pH}$ (units) & 1 & 0.1 & 1 & -- & -- & -- & -- & 5.9 \\
\hline Temperature, water $\left({ }^{\circ} \mathrm{C}\right)$ & 1 & 0.5 & 1 & -- & -- & -- & -- & 22.4 \\
\hline Turbidity (NTU) & 1 & 0.1 & 1 & -- & -- & -- & -- & 0.90 \\
\hline Oxygen dissolved (mg/L) & 1 & 0.1 & 1 & -- & -- & -- & -- & 6.8 \\
\hline $\begin{array}{l}\text { Fecal coliform } 0.7 \mu \mathrm{m}-\mathrm{MF} \\
\text { (cols. } / 100 \mathrm{~mL} \text { ) }\end{array}$ & 1 & 0 & 1 & -- & -- & -- & -- & 37 \\
\hline $\begin{array}{l}\text { Solids, residue at } 105^{\circ} \mathrm{C}, \\
\text { suspended }(\mathrm{mg} / \mathrm{L})\end{array}$ & 1 & 1 & 0 & -- & -- & -- & -- & -- \\
\hline Carbon, organic, total $(\mathrm{mg} / \mathrm{L})$ & 1 & 0.1 & 1 & -- & -- & -- & -- & 1.8 \\
\hline Oil and grease, total (mg/L) & 5 & 1 & 0 & -- & -- & -- & -- & -- \\
\hline \multicolumn{9}{|c|}{ During construction, April 1983 to June 1992, physical, biological, and organic } \\
\hline Discharge, inst., $\left(\mathrm{ft}^{3} / \mathrm{s}\right)$ & 75 & 0 & 75 & 0.07 & 0.42 & 0.80 & 1.2 & 21 \\
\hline $\begin{array}{l}\text { Specific conductance } \\
\left(\mathrm{uS} / \mathrm{cm} \text { at } 25^{\circ} \mathrm{C}\right)\end{array}$ & & 1 & & & & & & \\
\hline $\begin{array}{l}\left(\mu \mathrm{S} / \mathrm{cm} \text { at } 25^{\circ} \mathrm{C}\right) \\
\mathrm{pH} \text { (units) }\end{array}$ & $\begin{array}{l}13 \\
73\end{array}$ & $\begin{array}{l}1 \\
0.1\end{array}$ & $\begin{array}{l}73 \\
73\end{array}$ & 5.7 & $\begin{array}{r}200 \\
6.5\end{array}$ & $\begin{array}{r}220 \\
6.6\end{array}$ & $\begin{array}{r}238 \\
6.8\end{array}$ & $\begin{array}{r}360 \\
7.9\end{array}$ \\
\hline Temperature, water $\left({ }^{\circ} \mathrm{C}\right)$ & 75 & 0.5 & 75 & 20.0 & 23.0 & 24.0 & 25.0 & 27.5 \\
\hline Turbidity (NTU) & 63 & 0.1 & 63 & 0.90 & 1.6 & 3.0 & 12 & 360 \\
\hline Oxygen dissolved (mg/L) & 64 & 0.1 & 64 & 4.0 & 5.4 & 6.2 & 6.9 & 8.6 \\
\hline $\begin{array}{l}\text { Fecal coliform } 0.7 \mu \mathrm{m}-\mathrm{MF} \\
(\text { cols. } / 100 \mathrm{~mL})\end{array}$ & 31 & 0 & 31 & 23 & 230 & 620 & 2,800 & 9,000 \\
\hline $\begin{array}{l}\text { Solids, residue at } 105^{\circ} \mathrm{C}, \\
\text { suspended }(\mathrm{mg} / \mathrm{L})\end{array}$ & 41 & 1 & 32 & $<1$ & e1 & e5 & e23 & 500 \\
\hline Carbon, organic, total $(\mathrm{mg} / \mathrm{L})$ & L) 15 & 0.1 & 15 & 0.5 & 0.7 & 0.9 & 1.2 & 9.5 \\
\hline Oil and grease, total $(\mathrm{mg} / \mathrm{L})$ & 15 & 1 & 2 & $<1$ & -- & -- & -- & 3.0 \\
\hline \multicolumn{9}{|c|}{ After construction, July 1992 to January 1998, physical, biological, and organic } \\
\hline Discharge, inst., $\left(\mathrm{ft}^{3} / \mathrm{s}\right)$ & 57 & 0 & 57 & 0.13 & 0.27 & 0.45 & 0.83 & 8 \\
\hline $\begin{array}{l}\text { Specific conductance } \\
\left(\mathrm{uS} / \mathrm{cm} \text { at } 25^{0} \mathrm{C}\right)\end{array}$ & 57 & 1 & 57 & 80 & 208 & 220 & 234 & 295 \\
\hline $\mathrm{pH}$ (units) & 57 & 0.1 & 57 & 6.0 & 6.6 & 6.6 & 6.8 & 7.9 \\
\hline Temperature, water $\left({ }^{\circ} \mathrm{C}\right)$ & 57 & 0.5 & 57 & 21.0 & 23.0 & 23.5 & 24.0 & 25.5 \\
\hline Turbidity (NTU) & 56 & 0.1 & 56 & 0.30 & 0.80 & 1.3 & 2.0 & 30 \\
\hline Oxygen dissolved (mg/L) & 57 & 0.1 & 57 & 3.1 & 4.8 & 5.2 & 5.7 & 8.4 \\
\hline $\begin{array}{l}\text { Fecal coliform } 0.7 \mu \mathrm{m}-\mathrm{MF} \\
(\text { cols. } / 100 \mathrm{~mL})\end{array}$ & 23 & 0 & 23 & 160 & 260 & 590 & 1,500 & 8,600 \\
\hline $\begin{array}{l}\text { Solids, residue at } 105^{\circ} \mathrm{C}, \\
\text { suspended }(\mathrm{mg} / \mathrm{L})\end{array}$ & 23 & 1 & 13 & $<1$ & $\mathrm{e} 0.57$ & e3.0 & e 4.0 & 50 \\
\hline Carbon, organic, total $(\mathrm{mg} / \mathrm{L})$ & L) 12 & 0.1 & 12 & 0.4 & 0.6 & 1.4 & 1.8 & 5.2 \\
\hline Oil and grease, total (mg/L) & 12 & 1 & 1 & $<1$ & -- & -- & -- & 2 \\
\hline
\end{tabular}


Table 14. Statistical summary of selected water-quality data collected at station 16265600 , Right Branch Kamooalii Stream near Kaneohe, Oahu, Hawaii, from February 1983 to January 1998--Continued

\begin{tabular}{|c|c|c|c|c|c|c|c|c|}
\hline \multirow[b]{2}{*}{ Property or constituent } & \multirow{2}{*}{$\begin{array}{l}\text { Number } \\
\text { of } \\
\text { samples }\end{array}$} & \multirow{2}{*}{$\begin{array}{l}\text { Minimum } \\
\text { detection } \\
\text { limit }\end{array}$} & \multirow{2}{*}{$\begin{array}{l}\text { Number } \\
\text { of } \\
\text { detections }\end{array}$} & \multirow{2}{*}{$\begin{array}{l}\text { Mini- } \\
\text { mum }\end{array}$} & \multicolumn{3}{|c|}{ Concentration percentile } & \multirow{2}{*}{$\begin{array}{l}\text { Maxi- } \\
\text { mum }\end{array}$} \\
\hline & & & & & 25 & 50 & 75 & \\
\hline \multicolumn{9}{|c|}{ Before construction, February 1983 major ions and nutrients $(\mathrm{mg} / \mathrm{L})$} \\
\hline Hardness, total $\left(\right.$ as $\left.\mathrm{CaCO}_{3}\right)$ & 1 & 1 & 1 & -- & -- & -- & -- & 54 \\
\hline Calcium, dissolved & 1 & 0.02 & 1 & -- & -- & -- & -- & 11.0 \\
\hline Magnesium, dissolved & 1 & 0.01 & 0 & -- & -- & -- & -- & 6.5 \\
\hline Sodium, dissolved & 1 & 0.2 & 1 & -- & -- & -- & -- & 16 \\
\hline Potassium, dissolved & 1 & 0.1 & 1 & -- & -- & -- & -- & 0.9 \\
\hline Alkalinity $\left(\right.$ as $\left.\mathrm{CaCO}_{3}\right)$ & 1 & 1 & 1 & -- & -- & -- & -- & 54 \\
\hline Sulfate, dissolved & 1 & 0.1 & 1 & -- & -- & -- & -- & 7.0 \\
\hline Chloride, dissolved & 1 & 0.1 & 1 & -- & -- & -- & -- & 19 \\
\hline Fluoride, dissolved & 1 & 0.1 & 0 & -- & -- & -- & -- & -- \\
\hline Silica, dissolved & 1 & 0.01 & 1 & -- & -- & -- & -- & 18 \\
\hline $\begin{array}{l}\text { Nitrogen, nitrite plus } \\
\text { nitrate, total (as N) }\end{array}$ & 1 & 0.05 & 1 & -- & -- & -- & -- & 0.4 \\
\hline Nitrogen, ammonia & & & & & & & & \\
\hline plus organic, total (as N) & 1 & 0.2 & 1 & -- & -- & -- & -- & 0.70 \\
\hline Phosphorus, total (as P) & 1 & 0.01 & 1 & -- & -- & -- & -- & 0.01 \\
\hline \multicolumn{9}{|c|}{ During construction, April 1983 to June 1992, major ions and nutrients $(\mathrm{mg} / \mathrm{L})$} \\
\hline Hardness, total (as $\left.\mathrm{CaCO}_{3}\right)$ & 15 & 1 & 15 & 21 & 62 & 67 & 87 & 100 \\
\hline Calcium, dissolved & 15 & 0.02 & 15 & 4.9 & 13 & 14 & 19 & 31 \\
\hline Magnesium, dissolved & 15 & 0.01 & 15 & 2.2 & 6.9 & 7.5 & 8.5 & 13 \\
\hline Sodium, dissolved & 15 & 0.2 & 15 & 5.3 & 16 & 18 & 20 & 24 \\
\hline Potassium, dissolved & 15 & 0.1 & 15 & 0.9 & 0.9 & 1 & 2 & 8 \\
\hline Alkalinity $\left(\right.$ as $\left.\mathrm{CaCO}_{3}\right)$ & 15 & 1 & 15 & 17 & 52 & 57 & 68 & 94 \\
\hline Sulfate, dissolved & 15 & 0.1 & 15 & 10 & 12 & 16 & 21 & 44 \\
\hline Chloride, dissolved & 15 & 0.1 & 15 & 5.2 & 19 & 20 & 22 & 24 \\
\hline Fluoride, dissolved & 15 & 0.1 & 4 & $<0.1$ & -- & -- & -- & 0.30 \\
\hline Silica, dissolved & 15 & 0.01 & 15 & 3.0 & 18 & 20 & 22 & 24 \\
\hline $\begin{array}{l}\text { Nitrogen, nitrite plus } \\
\text { nitrate, total (as N) }\end{array}$ & 38 & 0.05 & 38 & 0.2 & 0.40 & 0.50 & 0.60 & 2.6 \\
\hline Nitrogen, ammonia & & & & & & & & \\
\hline plus organic, total (as N) & 38 & 0.2 & 26 & $<0.20$ & $\mathrm{e} 0.10$ & $\mathrm{e} 0.30$ & $\mathrm{e} 0.40$ & 2.6 \\
\hline Phosphorus, total (as P) & 38 & 0.01 & 34 & $<0.01$ & e0.02 & $\mathrm{e} 0.03$ & $\mathrm{e} 0.06$ & 0.58 \\
\hline \multicolumn{9}{|c|}{ After construction, July 1992 to January 1998, major ions and nutrients (mg/L) } \\
\hline Hardness, total (as $\left.\mathrm{CaCO}_{3}\right)$ & 12 & 1 & 12 & 52 & 60 & 66 & 74 & 76 \\
\hline Calcium, dissolved & 12 & 0.02 & 12 & 11 & 12 & 13 & 15 & 16 \\
\hline Magnesium, dissolved & 12 & 0.01 & 12 & 5.9 & 7.2 & 7.8 & 8.4 & 9.3 \\
\hline Sodium, dissolved & 12 & 0.2 & 12 & 15 & 17 & 18 & 19 & 19 \\
\hline Potassium, dissolved & 12 & 0.1 & 12 & 0.9 & 1.1 & 1.2 & 1.6 & 1.7 \\
\hline Alkalinity $\left(\right.$ as $\mathrm{CaCO}_{3}$ ) & 12 & 1 & 12 & 50 & 57 & 61 & 67 & 74 \\
\hline Sulfate, dissolved & 12 & 0.1 & 12 & 9.9 & 12 & 15 & 17 & 18 \\
\hline Chloride, dissolved & 12 & 0.1 & 12 & 19 & 19 & 21 & 22 & 24 \\
\hline Fluoride, dissolved & 12 & 0.1 & 1 & $<0.1$ & -- & -- & -- & 0.1 \\
\hline Silica, dissolved & 12 & 0.01 & 12 & 16 & 17 & 19 & 21 & 23 \\
\hline $\begin{array}{l}\text { Nitrogen, nitrite plus } \\
\text { nitrate, total (as N) }\end{array}$ & 2 & 0.05 & 2 & $<0.1$ & -- & -- & -- & 0.55 \\
\hline $\begin{array}{l}\text { Nitrogen, nitrite plus } \\
\text { nitrate, dissolved (as N) }\end{array}$ & 17 & 0.05 & 17 & 0.32 & 0.38 & 0.45 & 0.50 & 0.55 \\
\hline $\begin{array}{l}\text { Nitrogen, ammonia } \\
\text { plus organic, total (as N) }\end{array}$ & 23 & 0.1 & 1 & $<0.2$ & -- & -- & -- & 0.5 \\
\hline Phosphorus, total (as P) & 23 & 0.05 & 17 & $<0.01$ & $\mathrm{e} 0.003$ & $\mathrm{e} 0.01$ & $\mathrm{e} 0.02$ & 0.28 \\
\hline
\end{tabular}


Table 14. Statistical summary of selected water-quality data collected at station 16265600, Right Branch Kamooalii Stream near Kaneohe, Oahu, Hawaii, from February 1983 to January 1998--Continued

\begin{tabular}{|c|c|c|c|c|c|c|c|c|}
\hline \multirow[b]{2}{*}{ Property or constituent } & \multirow{2}{*}{$\begin{array}{l}\text { Number } \\
\text { of } \\
\text { samples }\end{array}$} & \multirow{2}{*}{$\begin{array}{l}\text { Minimum } \\
\text { detection } \\
\text { limit }\end{array}$} & \multirow{2}{*}{$\begin{array}{l}\text { Number } \\
\text { of } \\
\text { detections }\end{array}$} & \multirow{2}{*}{$\begin{array}{l}\text { Mini- } \\
\text { mum }\end{array}$} & \multicolumn{3}{|c|}{ Concentration percentile } & \multirow{2}{*}{$\begin{array}{l}\text { Maxi- } \\
\text { mum }\end{array}$} \\
\hline & & & & & 25 & 50 & 75 & \\
\hline \multicolumn{9}{|c|}{ Before construction, February 1983 trace metals $(\mu \mathrm{g} / \mathrm{L})$} \\
\hline Aluminum, total recoverable & e 1 & 10 & 1 & -- & -- & -- & -- & 90 \\
\hline Aluminum, dissolved & 1 & 10 & 1 & -- & -- & -- & -- & 10 \\
\hline Cadmium, total recoverable & 1 & 1 & 0 & -- & -- & -- & -- & -- \\
\hline Cadmium, dissolved & 1 & 1 & 0 & -- & -- & -- & -- & -- \\
\hline Chromium, total recoverable & 1 & 10 & 0 & -- & -- & -- & -- & -- \\
\hline Chromium, dissolved & 1 & 1 & 0 & -- & -- & -- & -- & -- \\
\hline Copper, total recoverable & 1 & 1 & 1 & -- & -- & -- & -- & 1 \\
\hline Copper, dissolved & 1 & 1 & 1 & -- & -- & -- & -- & 1 \\
\hline Iron, total recoverable & 1 & 10 & 1 & -- & -- & -- & -- & 450 \\
\hline Iron, dissolved & 1 & 3 & 1 & -- & -- & -- & -- & 270 \\
\hline Lead, total recoverable & 1 & 1 & 1 & -- & -- & -- & -- & 1 \\
\hline Lead, dissolved & 1 & 1 & 1 & -- & -- & -- & -- & 1 \\
\hline Nickel, total recoverable & 1 & 1 & 1 & -- & -- & -- & -- & 5 \\
\hline Nickel, dissolved & 1 & 1 & 1 & -- & -- & -- & -- & 3 \\
\hline Zinc, total recoverable & 1 & 10 & 1 & -- & -- & -- & -- & 40 \\
\hline Zinc, dissolved & 1 & 3 & 1 & -- & -- & -- & -- & 33 \\
\hline \multicolumn{9}{|c|}{ During construction, April 1983 to June 1992, trace metals $(\mu \mathrm{g} / \mathrm{L})$} \\
\hline Aluminum, total recoverable & e 15 & 10 & 15 & 40 & 140 & 200 & 520 & 5,400 \\
\hline Aluminum, dissolved & 15 & 10 & 5 & $<10$ & $\mathrm{e} 0.26$ & $\mathrm{e} 1.5$ & e10 & 110 \\
\hline Cadmium, total recoverable & 15 & 1 & 1 & $<1$ & -- & -- & -- & 3 \\
\hline Cadmium, dissolved & 14 & 1 & 0 & -- & -- & -- & -- & -- \\
\hline Chromium, total recoverable & e 15 & 1 & 10 & $<1$ & e1 & e2 & e10 & 80 \\
\hline Chromium, dissolved & 14 & 1 & 2 & $<1$ & -- & -- & -- & 10 \\
\hline Copper, total recoverable & 15 & 1 & 15 & 2 & 2 & 4 & 5 & 60 \\
\hline Copper, dissolved & 14 & 1 & 14 & 1 & 1 & 2 & 4 & 8 \\
\hline Iron, total recoverable & 15 & 10 & 15 & 270 & 520 & 720 & 1,700 & 39,000 \\
\hline Iron, dissolved & 15 & 3 & 15 & 10 & 40 & 80 & 100 & 170 \\
\hline Lead, total recoverable & 15 & 1 & 12 & $<1$ & e1 & e2 & e3 & 31 \\
\hline Lead, dissolved & 14 & 1 & 8 & $<1$ & $\mathrm{e} 0.46$ & $\mathrm{e} 1.0$ & $\mathrm{e} 1.2$ & 4.0 \\
\hline Nickel, total recoverable & 15 & 1 & 15 & 1 & 3 & 9 & 17 & 120 \\
\hline Nickel, dissolved & 15 & 1 & 11 & $<1$ & $\mathrm{e} 0.80$ & $\mathrm{e} 2.0$ & e4.0 & $\mathrm{e} 8.0$ \\
\hline Zinc, total recoverable & 15 & 10 & 14 & $<10$ & e40 & $\mathrm{e} 50$ & e140 & 170 \\
\hline Zinc, dissolved & 14 & 3 & 13 & $<3$ & e13 & e22 & e54 & 160 \\
\hline \multicolumn{9}{|c|}{ After construction, July 1992 to January 1998 , trace metals $(\mu \mathrm{g} / \mathrm{L})$} \\
\hline Aluminum, total recoverable & e 12 & 10 & 11 & $<10$ & $\mathrm{e} 20$ & e50 & e90 & 1,100 \\
\hline Aluminum, dissolved & 12 & 10 & 3 & $<5$ & -- & -- & -- & 30 \\
\hline Cadmium, total recoverable & 12 & 1 & 0 & -- & -- & -- & -- & -- \\
\hline Cadmium, dissolved & -- & 1 & -- & -- & -- & -- & -- & -- \\
\hline Chromium, total recoverable & e 12 & 1 & 6 & $<1$ & $\mathrm{e} 0.16$ & $\mathrm{e} 0.44$ & $\mathrm{e} 1.8$ & 5.0 \\
\hline Chromium, dissolved & -- & 1 & -- & -- & -- & -- & -- & -- \\
\hline Copper, total recoverable & 12 & 1 & 6 & $<1$ & $\mathrm{e} 0.30$ & $\mathrm{e} 0.67$ & $\mathrm{e} 2.0$ & 5.0 \\
\hline Copper, dissolved & -- & 1 & -- & -- & -- & -- & -- & -- \\
\hline Iron, total recoverable & 12 & 10 & 12 & 340 & 400 & 460 & 720 & 4,600 \\
\hline Iron, dissolved & 12 & 3 & 12 & 36 & 86 & 100 & 130 & 290 \\
\hline Lead, total recoverable & 12 & 1 & 2 & $<1$ & -- & -- & -- & 3 \\
\hline Lead, dissolved & -- & 1 & -- & -- & -- & -- & -- & -- \\
\hline Nickel, total recoverable & 12 & 1 & 12 & 1 & 1 & 2 & 2 & 6 \\
\hline Nickel, dissolved & 12 & 1 & 6 & $<1$ & $\mathrm{e} 0.41$ & $\mathrm{e} 0.65$ & $\mathrm{e} 1.0$ & 2.0 \\
\hline Zinc, total recoverable & 12 & 10 & 9 & $<10$ & e7.4 & e10 & e30 & 30 \\
\hline Zinc, dissolved & -- & 20 & -- & -- & -- & -- & -- & -- \\
\hline
\end{tabular}


Table 15. Statistical summary of selected water-quality data collected at station 16265700, Kamooalii Stream at alt $200 \mathrm{ft}$. near Kaneohe,Oahu, Hawaii, from February 1983 to January 1998

[inst., instantaneous; $\mu \mathrm{S} / \mathrm{cm}$ at $25^{\circ} \mathrm{C}$, microsiemens per centimeter at 25 degrees Celsius; $\mathrm{mg} / \mathrm{L}$, milligram per liter; $\mu \mathrm{g} / \mathrm{L}$, microgram per liter; <, less than; e, estimated using log-probability regression; $0.7 \mu \mathrm{m}-\mathrm{MF}, 0.7$ micron membrane filter; cols./100 mL, colonies per 100 milliliters; $\mathrm{ft}^{3} / \mathrm{s}$, cubic foot per second; ${ }^{\circ} \mathrm{C}$, degrees Celsius; NTU, nephelometric turbidity unit; --, no data or not computed; Range of dates are the time periods of water-quality data collection during the specified construction activity]

\begin{tabular}{|c|c|c|c|c|c|c|c|c|}
\hline \multirow[b]{2}{*}{ Property or constituent } & \multirow{2}{*}{$\begin{array}{l}\text { Number } \\
\text { of } \\
\text { samples }\end{array}$} & \multirow{2}{*}{$\begin{array}{l}\text { Minimum } \\
\text { detection } \\
\text { limit }\end{array}$} & \multirow{2}{*}{$\begin{array}{l}\text { Number } \\
\text { of } \\
\text { detections }\end{array}$} & \multirow{2}{*}{$\begin{array}{l}\text { Mini- } \\
\text { mum }\end{array}$} & \multicolumn{3}{|c|}{ Concentration percentile } & \multirow{2}{*}{$\begin{array}{l}\text { Maxi- } \\
\text { mum }\end{array}$} \\
\hline & & & & & 25 & 50 & 75 & \\
\hline \multicolumn{9}{|c|}{ Before construction, February 1983 to June 1989, physical, biological, and nutrients } \\
\hline Discharge, inst., $\left(\mathrm{ft}^{3} / \mathrm{s}\right)$ & 19 & 0 & 19 & 0.05 & 0.40 & 0.85 & 2.8 & 4.7 \\
\hline Specific conductance & & & & & & & & \\
\hline$\left(\mu \mathrm{S} / \mathrm{cm}\right.$ at $\left.25^{\circ} \mathrm{C}\right)$ & 19 & 1 & 19 & 159 & 193 & 200 & 207 & 212 \\
\hline $\mathrm{pH}$ (units) & 19 & 0.1 & 19 & 6.0 & 6.5 & 6.8 & 7.0 & 8.6 \\
\hline Temperature, water $\left({ }^{\circ} \mathrm{C}\right)$ & 19 & 0.5 & 19 & 20.0 & 21.5 & 22.5 & 22.5 & 26.0 \\
\hline Turbidity (NTU) & 18 & 0.1 & 18 & 0.40 & 0.50 & 0.95 & 1.4 & 9.0 \\
\hline Oxygen dissolved (mg/L) & 18 & 0.1 & 18 & 6.0 & 7.2 & 7.8 & 8.3 & 9.4 \\
\hline $\begin{array}{l}\text { Fecal coliform } 0.7 \mu \mathrm{m}-\mathrm{MF} \\
(\text { cols. } / 100 \mathrm{~mL})\end{array}$ & 10 & 0 & 10 & 33 & 95 & 320 & 1,007 & 2,000 \\
\hline $\begin{array}{l}\text { Solids, residue at } 105^{\circ} \mathrm{C}, \\
\text { suspended }(\mathrm{mg} / \mathrm{L})\end{array}$ & 14 & 1 & 13 & $<1$ & $\mathrm{e} 2.0$ & $\mathrm{e} 4.5$ & e10 & 18 \\
\hline $\begin{array}{l}\text { Nitrogen, nitrite plus } \\
\text { nitrate, total (as N) }\end{array}$ & 11 & 0.1 & 11 & 0.20 & 0.20 & 0.20 & 0.30 & 0.30 \\
\hline $\begin{array}{l}\text { Nitrogen, ammonia } \\
\text { plus organic, total (as } \mathrm{N} \text { ) }\end{array}$ & 11 & 0.2 & 10 & $<0.2$ & $\mathrm{e} 0.20$ & $\mathrm{e} 0.40$ & $e 0.50$ & 1.1 \\
\hline Phosphorus, total (as P) & 11 & 0.01 & 11 & 0.01 & 0.02 & 0.03 & 0.04 & 0.07 \\
\hline \multicolumn{9}{|c|}{ During construction, June 1989 to June 1992, physical, biological, and nutrients } \\
\hline Discharge, inst., $\left(\mathrm{ft}^{3} / \mathrm{s}\right)$ & 34 & 0 & 34 & 0.15 & 0.61 & 1.3 & 2.3 & 39 \\
\hline Specific conductance & & & & & & & & \\
\hline$\left(\mu \mathrm{S} / \mathrm{cm}\right.$ at $\left.25^{\circ} \mathrm{C}\right)$ & 33 & 1 & 33 & 148 & 204 & 220 & 230 & 380 \\
\hline $\mathrm{pH}$ (units) & 33 & 0.1 & 33 & 6.4 & 6.9 & 7.0 & 7.4 & 8.1 \\
\hline Temperature, water $\left({ }^{\circ} \mathrm{C}\right)$ & 33 & 0.5 & 33 & 20.5 & 21.2 & 22.0 & 23.0 & 24.0 \\
\hline Turbidity (NTU) & 34 & 0.1 & 34 & 0.40 & 0.98 & 5.6 & 78 & 13,000 \\
\hline Oxygen dissolved (mg/L) & 31 & 0.1 & 31 & 5.8 & 7.4 & 7.7 & 7.9 & 8.8 \\
\hline $\begin{array}{l}\text { Fecal coliform } 0.7 \mu \mathrm{m}-\mathrm{MF} \\
\text { (cols. } / 100 \mathrm{~mL})\end{array}$ & 12 & 0 & 12 & 64 & 135 & 480 & 2,625 & 12,000 \\
\hline $\begin{array}{l}\text { Solids, residue at } 105^{\circ} \mathrm{C}, \\
\text { suspended }(\mathrm{mg} / \mathrm{L})\end{array}$ & 13 & 1 & 10 & $<1$ & e2.3 & $\mathrm{e} 5.0$ & e46 & 404 \\
\hline $\begin{array}{l}\text { Nitrogen, nitrite plus } \\
\text { nitrate, total (as N) }\end{array}$ & 13 & 0.1 & 13 & 0.10 & 0.19 & 0.26 & 0.45 & 0.84 \\
\hline $\begin{array}{l}\text { Nitrogen, nitrite plus } \\
\text { nitrate, dissolved (as N) }\end{array}$ & 0 & 0.05 & -- & -- & -- & -- & -- & -- \\
\hline $\begin{array}{l}\text { Nitrogen, ammonia } \\
\text { plus organic, total (as N) }\end{array}$ & 13 & 0.2 & 6 & $<0.2$ & $\mathrm{e} 0.09$ & $\mathrm{e} 0.15$ & $\mathrm{e} 0.30$ & 0.50 \\
\hline Phosphorus, total (as P) & 13 & 0.01 & 9 & $<0.01$ & $\mathrm{e} 0.01$ & $\mathrm{e} 0.03$ & $\mathrm{e} 0.24$ & 0.36 \\
\hline
\end{tabular}


Table 15. Statistical summary of selected water-quality data collected at station 16265700 , Kamooalii Stream at alt 200 ft near Kaneohe, Oahu, Hawaii, from February 1983 to January 1998--Continued

\begin{tabular}{|c|c|c|c|c|c|c|c|c|}
\hline \multirow[b]{2}{*}{ Property or constituent } & \multirow{2}{*}{$\begin{array}{l}\text { Number } \\
\text { of } \\
\text { samples }\end{array}$} & \multirow{2}{*}{$\begin{array}{l}\text { Minimum } \\
\text { detection } \\
\text { limit }\end{array}$} & \multirow{2}{*}{$\begin{array}{l}\text { Number } \\
\text { of } \\
\text { detections }\end{array}$} & \multirow{2}{*}{$\begin{array}{l}\text { Mini- } \\
\text { mum }\end{array}$} & \multicolumn{3}{|c|}{ Concentration percentile } & \multirow{2}{*}{$\begin{array}{l}\text { Maxi- } \\
\text { mum }\end{array}$} \\
\hline & & & & & 25 & 50 & 75 & \\
\hline \multicolumn{9}{|c|}{ After construction, July 1992 to January 1998, physical, biological, and nutrients } \\
\hline Discharge, inst., $\left(\mathrm{ft}^{3} / \mathrm{s}\right)$ & 47 & 0 & 47 & 0.12 & 0.32 & 0.68 & 1.2 & 3.2 \\
\hline Specific conductance & & & & & & & & \\
\hline$\left(\mu \mathrm{S} / \mathrm{cm}\right.$ at $\left.25^{\circ} \mathrm{C}\right)$ & 47 & 1 & 47 & 23 & 215 & 223 & 232 & 267 \\
\hline $\mathrm{pH}$ (units) & 47 & 0.1 & 47 & 4.5 & 6.6 & 6.8 & 7.1 & 7.6 \\
\hline Temperature, water $\left({ }^{\circ} \mathrm{C}\right)$ & 47 & 0.5 & 47 & 20.0 & 21.5 & 22.0 & 22.5 & 24.5 \\
\hline Turbidity (NTU) & 47 & 0.1 & 47 & 0.10 & 0.30 & 0.60 & 1.0 & 2.5 \\
\hline Oxygen dissolved (mg/L) & 46 & 0.1 & 46 & 4.3 & 6.4 & 7.4 & 8.1 & 8.8 \\
\hline $\begin{array}{l}\text { Fecal coliform } 0.7 \mu \mathrm{m}-\mathrm{MF} \\
(\text { cols } . / 100 \mathrm{~mL})\end{array}$ & 19 & 0 & 19 & 27 & 80 & 180 & 500 & 2,500 \\
\hline $\begin{array}{l}\text { Solids, residue at } 105^{\circ} \mathrm{C}, \\
\text { suspended }(\mathrm{mg} / \mathrm{L})\end{array}$ & 19 & 1 & 13 & $<1$ & $\mathrm{e} 0.6$ & e1.0 & $\mathrm{e} 4.0$ & 8.0 \\
\hline $\begin{array}{l}\text { Nitrogen, nitrite plus } \\
\text { nitrate, total (as } N)\end{array}$ & 2 & 0.05 & 2 & 0.20 & -- & -- & -- & 0.30 \\
\hline $\begin{array}{l}\text { Nitrogen, nitrite plus } \\
\text { nitrate, dissolved (as N) }\end{array}$ & 12 & 0.05 & 12 & 0.16 & 0.24 & 0.33 & 0.54 & 0.59 \\
\hline $\begin{array}{l}\text { Nitrogen, ammonia } \\
\text { plus organic, total (as N) }\end{array}$ & 19 & 0.1 & 0 & -- & -- & -- & -- & -- \\
\hline Phosphorus, total (as P) & 19 & 0.05 & 16 & $<0.05$ & $\mathrm{e} 0.01$ & $\mathrm{e} 0.02$ & $\mathrm{e} 0.03$ & 0.06 \\
\hline
\end{tabular}


Table 16. Statistical summary of selected water-quality data collected at station 16266500 , Hooleinaiwa Stream at alt $220 \mathrm{ft}$ near Kaneohe, Oahu, Hawaii, from February 1983 to February 1997

[inst., instantaneous; $\mu \mathrm{S} / \mathrm{cm}$ at $25^{\circ} \mathrm{C}$, microsiemens per centimeter at 25 degrees Celsius; $\mathrm{mg} / \mathrm{L}$, milligram per liter; $\mu \mathrm{g} / \mathrm{L}$, microgram per liter; <, less than; e, estimated using log-probability regression; $0.7 \mu \mathrm{m}-\mathrm{MF}, 0.7$ micron membrane filter; cols./100 mL, colonies per 100 milliliters; $\mathrm{ft}^{3} / \mathrm{s}$, cubic foot per second; ${ }^{\circ} \mathrm{C}$, degrees Celsius; NTU, nephelometric turbidity unit; --, no data or not computed; Range of dates are the time periods of water-quality data collection during the specified construction activity]

\begin{tabular}{|c|c|c|c|c|c|c|c|c|}
\hline Property or constituent & $\begin{array}{l}\text { Number } \\
\text { of } \\
\text { samples }\end{array}$ & $\begin{array}{l}\text { Minimum } \\
\text { detection } \\
\text { limit }\end{array}$ & $\begin{array}{l}\text { Number } \\
\text { of } \\
\text { detections }\end{array}$ & $\begin{array}{l}\text { Mini- } \\
\text { mum }\end{array}$ & $\frac{\text { Concen }}{25}$ & $\frac{\text { tration per }}{50}$ & $\frac{\text { centile }}{75}$ & $\begin{array}{l}\text { Maxi- } \\
\text { mum }\end{array}$ \\
\hline \multicolumn{9}{|c|}{ Before construction, February 1983 to June 1989, physical, biological, and nutrients } \\
\hline Discharge, inst., $\left(\mathrm{ft}^{3} / \mathrm{s}\right)$ & 16 & 0 & 16 & 0.07 & 0.13 & 0.30 & 0.77 & 1.7 \\
\hline Specific conductance & & & & & & & & \\
\hline$\left(\mu \mathrm{S} / \mathrm{cm}\right.$ at $\left.25^{\circ} \mathrm{C}\right)$ & 16 & 1 & 16 & 99 & 113 & 121 & 125 & 133 \\
\hline $\mathrm{pH}$ (units) & 16 & 0.1 & 16 & 6.1 & 6.4 & 6.7 & 6.8 & 7.0 \\
\hline Temperature, water $\left({ }^{\circ} \mathrm{C}\right)$ & 16 & 0.5 & 16 & 21.0 & 22.3 & 22.5 & 23.5 & 25.0 \\
\hline Turbidity (NTU) & 15 & 0.1 & 15 & 0.30 & 0.30 & 0.70 & 1.0 & 2.4 \\
\hline Oxygen dissolved (mg/L) & 15 & 0.1 & 15 & 6.6 & 6.9 & 7.3 & 8.5 & 9.3 \\
\hline $\begin{array}{l}\text { Fecal coliform } 0.7 \mu \mathrm{m}-\mathrm{MF} \\
(\text { cols. } / 100 \mathrm{~mL})\end{array}$ & 10 & 0 & 10 & 50 & 85 & 125 & 252 & 290 \\
\hline $\begin{array}{l}\text { Solids, residue at } 105^{\circ} \mathrm{C}, \\
\text { suspended }(\mathrm{mg} / \mathrm{L})\end{array}$ & 13 & 1 & 9 & $<1$ & e1.1 & e5.0 & $\mathrm{e} 9.5$ & 31 \\
\hline $\begin{array}{l}\text { Nitrogen, nitrite plus } \\
\text { nitrate, total (as N) }\end{array}$ & 10 & 0.05 & 2 & $<0.1$ & -- & -- & -- & 0.10 \\
\hline $\begin{array}{l}\text { Nitrogen, ammonia } \\
\text { plus organic, total (as N) }\end{array}$ & 10 & 0.2 & 4 & $<0.2$ & -- & -- & -- & 0.60 \\
\hline Phosphorus, total (as P) & 9 & 0.01 & 5 & $<0.01$ & $\mathrm{e} 0.002$ & $\mathrm{e} 0.01$ & $\mathrm{e} 0.01$ & 0.07 \\
\hline \multicolumn{9}{|c|}{ During construction, June 1989 to June 1992, physical, biological, and nutrients } \\
\hline Discharge, inst., $\left(\mathrm{ft}^{3} / \mathrm{s}\right)$ & 31 & 0 & 31 & 0.06 & 0.17 & 0.28 & 0.77 & 6.2 \\
\hline $\begin{array}{l}\text { Specific conductance } \\
\left(\mu \mathrm{S} / \mathrm{cm} \text { at } 25^{\circ} \mathrm{C}\right)\end{array}$ & 30 & 1 & 30 & 110 & 129 & 151 & 160 & 190 \\
\hline $\mathrm{pH}$ (units) & 30 & 0.1 & 30 & 6.2 & 6.8 & 7.0 & 7.2 & 7.5 \\
\hline Temperature, water $\left({ }^{\circ} \mathrm{C}\right)$ & 30 & 0.5 & 30 & 20.0 & 22.0 & 22.5 & 23.0 & 24.5 \\
\hline Turbidity (NTU) & 32 & 0.1 & 32 & 0.20 & 0.40 & 0.65 & 1.5 & 4,700 \\
\hline Oxygen dissolved (mg/L) & 30 & 0.1 & 30 & 5.4 & 7.1 & 7.9 & 8.3 & 8.8 \\
\hline $\begin{array}{l}\text { Fecal coliform } 0.7 \mu \mathrm{m}-\mathrm{MF} \\
(\text { cols. } / 100 \mathrm{~mL})\end{array}$ & 11 & 0 & 11 & 20 & 82 & 180 & 840 & 3,100 \\
\hline $\begin{array}{l}\text { Solids, residue at } 105^{\circ} \mathrm{C}, \\
\text { suspended }(\mathrm{mg} / \mathrm{L})\end{array}$ & 12 & 1 & 5 & $<1$ & $\mathrm{e} 0.08$ & $\mathrm{e} 0.60$ & e8.2 & 37 \\
\hline $\begin{array}{l}\text { Nitrogen, nitrite plus } \\
\text { nitrate, total (as N) }\end{array}$ & 12 & 0.05 & 9 & $<0.1$ & $\mathrm{e} 0.08$ & $\mathrm{e} 0.13$ & $\mathrm{e} 0.27$ & 0.40 \\
\hline $\begin{array}{l}\text { Nitrogen, nitrite plus } \\
\text { nitrate, dissolved (as N) }\end{array}$ & 0 & 0.05 & -- & -- & -- & -- & -- & -- \\
\hline $\begin{array}{l}\text { Nitrogen, ammonia } \\
\text { plus organic, total (as N) }\end{array}$ & 12 & 0.2 & 3 & 0.2 & -- & 0.4 & -- & 0.6 \\
\hline Phosphorus, total (as P) & 12 & 0.01 & 5 & $<0.01$ & $\mathrm{e} 0.002$ & $\mathrm{e} 0.006$ & $\mathrm{e} 0.03$ & 0.07 \\
\hline
\end{tabular}


Table 16. Statistical summary of selected water-quality data collected at station 16266500 , Hooleinaiwa Stream at alt $200 \mathrm{ft}$ near Kaneohe, Oahu, Hawaii, from February 1983 to February 1997--Continued

\begin{tabular}{|c|c|c|c|c|c|c|c|c|}
\hline \multirow[b]{2}{*}{ Property or constituent } & \multirow{2}{*}{$\begin{array}{l}\text { Number } \\
\text { of } \\
\text { samples }\end{array}$} & \multirow{2}{*}{$\begin{array}{l}\text { Minimum } \\
\text { detection } \\
\text { limit }\end{array}$} & \multirow{2}{*}{$\begin{array}{l}\text { Number } \\
\text { of } \\
\text { detections }\end{array}$} & \multirow{2}{*}{$\begin{array}{l}\text { Mini- } \\
\text { mum }\end{array}$} & \multicolumn{3}{|c|}{$\underline{\text { Concentration percentile }}$} & \multirow{2}{*}{$\begin{array}{l}\text { Maxi- } \\
\text { mum }\end{array}$} \\
\hline & & & & & 25 & 50 & 75 & \\
\hline \multicolumn{9}{|c|}{ After construction, July 1992 to February 1997, physical, biological, and nutrients } \\
\hline Discharge, inst., $\left(\mathrm{ft}^{3} / \mathrm{s}\right)$ & 46 & 0 & 46 & 0.01 & 0.08 & 0.19 & 0.38 & 1.9 \\
\hline Specific conductance & & & & & & & & \\
\hline$\left(\mu \mathrm{S} / \mathrm{cm}\right.$ at $\left.25^{\circ} \mathrm{C}\right)$ & 46 & 1 & 46 & 140 & 155 & 161 & 170 & 250 \\
\hline pH (units) & 46 & 0.1 & 46 & 6.0 & 6.6 & 6.8 & 6.9 & 7.4 \\
\hline Temperature, water $\left({ }^{\circ} \mathrm{C}\right)$ & 45 & 0.5 & 45 & 20.0 & 21.8 & 22.5 & 23.0 & 24.5 \\
\hline Turbidity (NTU) & 46 & 0.1 & 46 & 0.20 & 0.30 & 0.40 & 0.72 & 36 \\
\hline Oxygen dissolved (mg/L) & 46 & 0.1 & 46 & 4.0 & 6.4 & 6.8 & 7.3 & 8.9 \\
\hline $\begin{array}{l}\text { Fecal coliform } 0.7 \mu \mathrm{m}-\mathrm{MF} \\
(\text { cols. } / 100 \mathrm{~mL})\end{array}$ & 17 & 0 & 17 & 7 & 30 & 90 & 175 & 17,000 \\
\hline $\begin{array}{l}\text { Solids, residue at } 105^{\circ} \mathrm{C}, \\
\text { suspended }(\mathrm{mg} / \mathrm{L})\end{array}$ & 18 & 1 & 9 & $<1$ & $\mathrm{e} 0.10$ & $\mathrm{e} 0.72$ & $\mathrm{e} 3.5$ & 86 \\
\hline $\begin{array}{l}\text { Nitrogen, nitrite plus } \\
\text { nitrate, total (as N) }\end{array}$ & 2 & 0.05 & 2 & 0.12 & -- & -- & -- & 0.25 \\
\hline $\begin{array}{l}\text { Nitrogen, nitrite plus } \\
\text { nitrate, dissolved (as N) }\end{array}$ & 12 & 0.05 & 10 & $<0.05$ & $\mathrm{e} 0.07$ & $\mathrm{e} 0.10$ & $\mathrm{e} 0.14$ & 0.18 \\
\hline $\begin{array}{l}\text { Nitrogen, ammonia } \\
\text { plus organic, total (as } \mathrm{N} \text { ) }\end{array}$ & 17 & 0.1 & 1 & -- & -- & -- & -- & 0.50 \\
\hline Phosphorus, total (as P) & 18 & 0.05 & 2 & -- & -- & -- & -- & 0.12 \\
\hline
\end{tabular}


Table 17. Statistical summary of selected water-quality data collected at station 16267500 , Hooleinaiwa Stream above confluence with Kamooalii Stream near Kaneohe, Oahu, Hawaii, from February 1983 to January 1998

[inst., instantaneous; $\mathrm{uS} / \mathrm{cm}$ at $25^{\circ} \mathrm{C}$, microsiemens per centimeter at 25 degrees Celsius; $\mathrm{mg} / \mathrm{L}$, milligram per liter; $\mu \mathrm{g} / \mathrm{L}$, microgram per liter; <, less than; e, estimated using log-probability regression; $0.7 \mu \mathrm{m}-\mathrm{MF}, 0.7$ micron membrane filter; cols./100 mL, colonies per 100 milliliters; $\mathrm{ft}^{3} / \mathrm{s}$, cubic foot per second; ${ }^{\circ} \mathrm{C}$, degrees Celsius; NTU, nephelometric turbidity unit; --, no data or not computed; Range of dates are the time periods of water-quality data collection during the specified construction activity]

\begin{tabular}{|c|c|c|c|c|c|c|c|c|}
\hline Property or constituent & $\begin{array}{l}\text { Number } \\
\text { of } \\
\text { samples }\end{array}$ & $\begin{array}{l}\text { Minimum } \\
\text { detection } \\
\text { limit }\end{array}$ & $\begin{array}{l}\text { Number } \\
\text { of } \\
\text { detections }\end{array}$ & $\begin{array}{l}\text { Mini- } \\
\text { mum }\end{array}$ & $\frac{\text { Conce }}{25}$ & $\frac{\text { tration pe }}{50}$ & $\frac{\text { centile }}{75}$ & $\begin{array}{l}\text { Maxi- } \\
\text { mum }\end{array}$ \\
\hline \multicolumn{9}{|c|}{ Before construction, February 1983 to June 1989 , physical, biological, and nutrients } \\
\hline Discharge, inst., $\left(\mathrm{ft}^{3} / \mathrm{s}\right)$ & 17 & 0 & 17 & 0.44 & 0.64 & 0.84 & 1.2 & 3.5 \\
\hline Specific conductance & & & & & & & & \\
\hline $\begin{array}{l}\left(\mu \mathrm{S} / \mathrm{cm} \text { at } 25^{\circ} \mathrm{C}\right) \\
\mathrm{pH} \text { (units) }\end{array}$ & $\begin{array}{l}17 \\
17\end{array}$ & $\begin{array}{l}1 \\
0.1\end{array}$ & $\begin{array}{l}17 \\
17\end{array}$ & $\begin{array}{r}115 \\
6.3\end{array}$ & $\begin{array}{r}147 \\
6.7\end{array}$ & $\begin{array}{r}166 \\
6.9\end{array}$ & $\begin{array}{c}174 \\
7.1\end{array}$ & $\begin{array}{l}188 \\
7.4\end{array}$ \\
\hline Temperature, water $\left({ }^{\circ} \mathrm{C}\right)$ & 17 & 0.5 & 17 & 21.6 & 23.0 & 24.5 & 25.2 & 30.0 \\
\hline Turbidity (NTU) & 17 & 0.1 & 17 & 2.0 & 2.5 & 2.7 & 3.6 & 12 \\
\hline Oxygen dissolved (mg/L) & 17 & 0.1 & 17 & 5.6 & 6.5 & 6.8 & 7.8 & 5.6 \\
\hline $\begin{array}{l}\text { Fecal coliform } 0.7 \mu \mathrm{m}-\mathrm{MF} \\
\text { (cols. } / 100 \mathrm{~mL} \text { ) }\end{array}$ & 10 & 0 & 10 & 10 & 28 & 86 & 232 & 3,300 \\
\hline $\begin{array}{l}\text { Solids, residue at } 105^{\circ} \mathrm{C}, \\
\text { suspended }(\mathrm{mg} / \mathrm{L})\end{array}$ & 14 & 1 & 11 & $<1$ & $\mathrm{e} 1.0$ & e6.5 & e10 & 13 \\
\hline $\begin{array}{l}\text { Nitrogen, nitrite plus } \\
\text { nitrate, total (as N) }\end{array}$ & 11 & 0.05 & 2 & -- & -- & -- & -- & 0.10 \\
\hline $\begin{array}{l}\text { Nitrogen, ammonia } \\
\text { plus organic, total (as N) }\end{array}$ & 11 & 0.2 & 6 & $<0.2$ & $\mathrm{e} 0.16$ & $\mathrm{e} 0.21$ & $\mathrm{e} 0.50$ & 0.70 \\
\hline Phosphorus, total (as P) & 11 & 0.01 & 8 & $<0.01$ & $\mathrm{e} 0.01$ & $\mathrm{e} 0.02$ & $\mathrm{e} 0.03$ & 0.03 \\
\hline \multicolumn{9}{|c|}{ During construction, June 1989 to June 1992, physical, biological, and nutrients } \\
\hline Discharge, inst., $\left(\mathrm{ft}^{3} / \mathrm{s}\right)$ & 30 & 0 & 30 & 0.35 & 0.62 & 0.75 & 1.1 & 150 \\
\hline $\begin{array}{l}\text { Specific conductance } \\
\left(\mu \mathrm{S} / \mathrm{cm} \text { at } 25^{\circ} \mathrm{C}\right)\end{array}$ & 30 & 1 & 30 & 65 & 154 & 178 & 195 & 203 \\
\hline $\mathrm{pH}$ (units) & 30 & 0.1 & 30 & 6.8 & 7.0 & 7.3 & 7.4 & 7.8 \\
\hline Temperature, water $\left({ }^{\circ} \mathrm{C}\right)$ & 30 & 0.5 & 30 & 20.0 & 22.0 & 23.5 & 24.1 & 26.0 \\
\hline Turbidity (NTU) & 31 & 0.1 & 31 & 0.50 & 3.4 & 4.3 & 15 & 550 \\
\hline Oxygen dissolved (mg/L) & 30 & 0.1 & 30 & 6.2 & 7.4 & 7.8 & 8.1 & 8.6 \\
\hline $\begin{array}{l}\text { Fecal coliform } 0.7 \mu \mathrm{m}-\mathrm{MF} \\
\text { (cols. } / 100 \mathrm{~mL})\end{array}$ & 11 & 0 & 11 & 36 & 78 & 210 & 490 & 9,300 \\
\hline $\begin{array}{l}\text { Solids, residue at } 105^{\circ} \mathrm{C}, \\
\text { suspended }(\mathrm{mg} / \mathrm{L})\end{array}$ & 12 & 1 & 11 & $<1$ & $\mathrm{e} 1.8$ & e12 & $\mathrm{e} 28$ & 168 \\
\hline $\begin{array}{l}\text { Nitrogen, nitrite plus } \\
\text { nitrate, total (as N) }\end{array}$ & 12 & 0.05 & 4 & $<0.05$ & -- & -- & -- & 0.30 \\
\hline $\begin{array}{l}\text { Nitrogen, nitrite plus } \\
\text { nitrate, dissolved (as N) }\end{array}$ & 0 & 0.05 & -- & -- & -- & -- & -- & -- \\
\hline $\begin{array}{l}\text { Nitrogen, ammonia } \\
\text { plus organic, total (as N) }\end{array}$ & 12 & 0.2 & 4 & $<0.2$ & -- & -- & -- & 0.6 \\
\hline Phosphorus, total (as P) & 12 & 0.01 & 7 & $<0.01$ & $\mathrm{e} 0.003$ & $\mathrm{e} 0.01$ & $\mathrm{e} 0.05$ & 0.12 \\
\hline
\end{tabular}


Table 17. Statistical summary of selected water-quality data collected at station 16267500 , Hooleinaiwa Stream above confluence with Kamooalii Stream near Kaneohe, Oahu, Hawaii, from February 1983 to January 1998--Continued

\begin{tabular}{|c|c|c|c|c|c|c|c|c|}
\hline \multirow[b]{2}{*}{ Property or constituent } & \multirow{2}{*}{$\begin{array}{l}\text { Number } \\
\text { of } \\
\text { samples }\end{array}$} & \multirow{2}{*}{$\begin{array}{l}\text { Minimum } \\
\text { detection } \\
\text { limit }\end{array}$} & \multirow{2}{*}{$\begin{array}{l}\text { Number } \\
\text { of } \\
\text { detections }\end{array}$} & \multirow{2}{*}{$\begin{array}{l}\text { Mini- } \\
\text { mum }\end{array}$} & \multicolumn{3}{|c|}{ Concentration percentile } & \multirow{2}{*}{$\begin{array}{l}\text { Maxi- } \\
\text { mum }\end{array}$} \\
\hline & & & & & 25 & 50 & 75 & \\
\hline \multicolumn{9}{|c|}{ After construction, July 1992 to January 1998, physical, biological, and nutrients } \\
\hline Discharge, inst., $\left(\mathrm{ft}^{3} / \mathrm{s}\right)$ & 48 & 0 & 48 & 0.08 & 0.45 & 0.62 & 0.93 & 3.7 \\
\hline Specific conductance & & & & & & & & \\
\hline$\left(\mu \mathrm{S} / \mathrm{cm}\right.$ at $\left.25^{\circ} \mathrm{C}\right)$ & 48 & 1 & 48 & 142 & 186 & 192 & 197 & 200 \\
\hline pH (units) & 48 & 0.1 & 48 & 6.3 & 7.0 & 7.2 & 7.4 & 7.6 \\
\hline Temperature, water $\left({ }^{\circ} \mathrm{C}\right)$ & 47 & 0.5 & 47 & 20.0 & 22.0 & 23.0 & 24.0 & 26.5 \\
\hline Turbidity (NTU) & 48 & 0.1 & 48 & 0.38 & 1.8 & 3.1 & 5.0 & 61 \\
\hline Oxygen dissolved (mg/L) & 48 & 0.1 & 48 & 5.4 & 6.9 & 7.3 & 7.8 & 8.8 \\
\hline $\begin{array}{l}\text { Fecal coliform } 0.7 \mu \mathrm{m}-\mathrm{MF} \\
\text { (cols. } / 100 \mathrm{~mL} \text { ) }\end{array}$ & 20 & 0 & 20 & 16 & 44 & 130 & 218 & 5,500 \\
\hline $\begin{array}{l}\text { Solids, residue at } 105^{\circ} \mathrm{C}, \\
\text { suspended }(\mathrm{mg} / \mathrm{L})\end{array}$ & 20 & 1 & 16 & $<1$ & e1.0 & $\mathrm{e} 4.0$ & e8.7 & 174 \\
\hline $\begin{array}{l}\text { Nitrogen, nitrite plus } \\
\text { nitrate, total (as N) }\end{array}$ & 2 & 0.05 & 1 & $<0.05$ & -- & -- & -- & 0.08 \\
\hline $\begin{array}{l}\text { Nitrogen, nitrite plus } \\
\text { nitrate, dissolved (as N) }\end{array}$ & 14 & 0.05 & 9 & $<0.05$ & $\mathrm{e} 0.04$ & $\mathrm{e} 0.06$ & e0.09 & 0.09 \\
\hline $\begin{array}{l}\text { Nitrogen, ammonia } \\
\text { plus organic, total (as N) }\end{array}$ & 20 & 0.1 & 1 & -- & -- & -- & -- & 0.30 \\
\hline Phosphorus, total (as P) & 20 & 0.05 & 10 & $<0.05$ & $\mathrm{e} 0.01$ & $\mathrm{e} 0.02$ & $\mathrm{e} 0.02$ & 0.09 \\
\hline
\end{tabular}


Table 18. Statistical summary of selected water-quality data collected at station 16269500 , Kuou Stream at alt $200 \mathrm{ft}$ near Kaneohe, Oahu, Hawaii, from February 1983 to January 1998

[inst., instantaneous; $\mu \mathrm{S} / \mathrm{cm}$ at $25^{\circ} \mathrm{C}$, microsiemens per centimeter at 25 degrees Celsius; $\mathrm{mg} / \mathrm{L}$, milligram per liter; $\mu \mathrm{g} / \mathrm{L}$, microgram per liter; <, less than; e, estimated using log-probability regression; $0.7 \mu \mathrm{m}-\mathrm{MF}, 0.7$ micron membrane filter; cols. $/ 100 \mathrm{~mL}$, colonies per 100 milliliters; $\mathrm{ft}^{3} / \mathrm{s}$, cubic foot per second; ${ }^{\circ} \mathrm{C}$, degrees Celsius; $\mathrm{NTU}$, nephelometric turbidity unit; --, no data or not computed; Range of dates are the time periods of water-quality data collection during the specified construction activity]

\begin{tabular}{|c|c|c|c|c|c|c|c|c|}
\hline Property or constituent & $\begin{array}{l}\text { Number } \\
\text { of } \\
\text { samples }\end{array}$ & $\begin{array}{l}\text { Minimum } \\
\text { detection } \\
\text { limit }\end{array}$ & $\begin{array}{l}\text { Number } \\
\text { of } \\
\text { detections }\end{array}$ & $\begin{array}{l}\text { Mini- } \\
\text { mum }\end{array}$ & $\frac{\text { Conce }}{25}$ & $\frac{\text { tration pe }}{50}$ & $\frac{\text { centile }}{75}$ & $\begin{array}{l}\text { Maxi- } \\
\text { mum }\end{array}$ \\
\hline \multicolumn{9}{|c|}{ Before construction, February 1983 to June 1989, physical, biological, and nutrients } \\
\hline Discharge, inst., $\left(\mathrm{ft}^{3} / \mathrm{s}\right)$ & 17 & 0 & 17 & 0.03 & 0.01 & 0.20 & 0.52 & 6.0 \\
\hline $\begin{array}{l}\text { Specific conductance } \\
\left(\mu \mathrm{S} / \mathrm{cm} \text { at } 25^{\circ} \mathrm{C}\right)\end{array}$ & 16 & 1 & 16 & 200 & 212 & 248 & 290 & 320 \\
\hline $\mathrm{pH}$ (units) & 17 & 0.1 & 17 & 6.0 & 6.8 & 6.9 & 7.0 & 7.4 \\
\hline Temperature, water $\left({ }^{\circ} \mathrm{C}\right)$ & 17 & 0.5 & 17 & 20.5 & 22.0 & 22.4 & 22.8 & 24.5 \\
\hline Turbidity (NTU) & 15 & 0.1 & 15 & 1.4 & 2.3 & 2.7 & 4.0 & 43 \\
\hline Oxygen dissolved (mg/L) & 16 & 0.1 & 16 & 5.0 & 5.5 & 7.0 & 7.9 & 9.4 \\
\hline $\begin{array}{l}\text { Fecal coliform } 0.7 \mu \mathrm{m}-\mathrm{MF} \\
(\text { cols. } / 100 \mathrm{~mL})\end{array}$ & 10 & 0 & 10 & 10 & 33 & 112 & 245 & 390 \\
\hline $\begin{array}{l}\text { Solids, residue at } 105^{\circ} \mathrm{C}, \\
\text { suspended }(\mathrm{mg} / \mathrm{L})\end{array}$ & 13 & 1 & 8 & $<1$ & $\mathrm{e} 2.3$ & e5.0 & e9.0 & 13 \\
\hline $\begin{array}{l}\text { Nitrogen, nitrite plus } \\
\text { nitrate, total (as N) }\end{array}$ & 10 & 0.05 & 7 & $<0.1$ & $\mathrm{e} 0.03$ & $\mathrm{e} 0.20$ & $\mathrm{e} 0.65$ & 4.1 \\
\hline $\begin{array}{l}\text { Nitrogen, ammonia } \\
\text { plus organic, total (as N) }\end{array}$ & 10 & 0.2 & 7 & $<0.2$ & $\mathrm{e} 0.17$ & $\mathrm{e} 0.35$ & $\mathrm{e} 0.52$ & 0.70 \\
\hline Phosphorus, total (as P) & 10 & 0.01 & 7 & $<0.01$ & $\mathrm{e} 0.002$ & $\mathrm{e} 0.01$ & $\mathrm{e} 0.02$ & 0.20 \\
\hline \multicolumn{9}{|c|}{ During construction, June 1989 to June 1992, physical, biological, and nutrients } \\
\hline Discharge, inst., $\left(\mathrm{ft}^{3} / \mathrm{s}\right)$ & 30 & 0 & 30 & 0.01 & 0.02 & 0.06 & 0.17 & 1.1 \\
\hline $\begin{array}{l}\text { Specific conductance } \\
\left(\mu \mathrm{S} / \mathrm{cm} \text { at } 25^{\circ} \mathrm{C}\right)\end{array}$ & 30 & 1 & 30 & 225 & 260 & 280 & 300 & 325 \\
\hline $\mathrm{pH}$ (units) & 29 & 0.1 & 29 & 6.6 & 7.0 & 7.0 & 7.4 & 7.9 \\
\hline Temperature, water $\left({ }^{\circ} \mathrm{C}\right)$ & 30 & 0.5 & 30 & 20.5 & 22.0 & 22.5 & 23.0 & 25.0 \\
\hline Turbidity (NTU) & 31 & 0.1 & 31 & 0.2 & 1.0 & 2.4 & 4.3 & 16 \\
\hline Oxygen dissolved (mg/L) & 30 & 0.1 & 30 & 5.7 & 7.0 & 7.4 & 8.2 & 8.7 \\
\hline $\begin{array}{l}\text { Fecal coliform } 0.7 \mu \mathrm{m}-\mathrm{MF} \\
\text { (cols. } / 100 \mathrm{~mL} \text { ) }\end{array}$ & 11 & 0 & 11 & 9.0 & 140 & 160 & 380 & 540 \\
\hline $\begin{array}{l}\text { Solids, residue at } 105^{\circ} \mathrm{C} \text {, } \\
\text { suspended }(\mathrm{mg} / \mathrm{L})\end{array}$ & 12 & 1 & 9 & $<1$ & e1.4 & $\mathrm{e} 4.0$ & e10 & 21 \\
\hline $\begin{array}{l}\text { Nitrogen, nitrite plus } \\
\text { nitrate, total (as N) }\end{array}$ & 12 & 0.05 & 12 & 0.10 & 0.20 & 0.28 & 0.48 & 0.70 \\
\hline $\begin{array}{l}\text { Nitrogen, nitrite plus } \\
\text { nitrate, dissolved (as N) }\end{array}$ & 0 & 0.05 & -- & -- & -- & -- & -- & -- \\
\hline $\begin{array}{l}\text { Nitrogen, ammonia } \\
\text { plus organic, total (as N) }\end{array}$ & 12 & 0.2 & 4 & $<0.2$ & -- & -- & -- & 0.70 \\
\hline Phosphorus, total (as P) & 12 & 0.01 & 7 & $<0.01$ & $\mathrm{e} 0.005$ & $\mathrm{e} 0.01$ & $\mathrm{e} 0.02$ & 0.04 \\
\hline
\end{tabular}


Table 18. Statistical summary of selected water-quality data collected at station 16269500 , Kuou Stream at alt $220 \mathrm{ft}$ near Kaneohe, Oahu, Hawaii, from February 1983 to January 1998--Continued

\begin{tabular}{|c|c|c|c|c|c|c|c|c|}
\hline \multirow[b]{2}{*}{ Property or constituent } & \multirow{2}{*}{$\begin{array}{l}\text { Number } \\
\text { of } \\
\text { samples }\end{array}$} & \multirow{2}{*}{$\begin{array}{l}\text { Minimum } \\
\text { detection } \\
\text { limit }\end{array}$} & \multirow{2}{*}{$\begin{array}{l}\text { Number } \\
\text { of } \\
\text { detections }\end{array}$} & \multirow{2}{*}{$\begin{array}{l}\text { Mini- } \\
\text { mum }\end{array}$} & \multicolumn{3}{|c|}{$\underline{\text { Concentration percentile }}$} & \multirow{2}{*}{$\begin{array}{l}\text { Maxi- } \\
\text { mum }\end{array}$} \\
\hline & & & & & 25 & 50 & 75 & \\
\hline \multicolumn{9}{|c|}{ After construction, July 1992 to January 1998, physical, biological, and nutrients } \\
\hline Discharge, inst., $\left(\mathrm{ft}^{3} / \mathrm{s}\right)$ & 43 & 0 & 43 & 0 & 0.02 & 0.03 & 0.08 & 1.0 \\
\hline Specific conductance & & & & & & & & \\
\hline$\left(\mu \mathrm{S} / \mathrm{cm}\right.$ at at $\left.25^{\circ} \mathrm{C}\right)$ & 45 & 1 & 45 & 215 & 304 & 318 & 350 & 400 \\
\hline $\mathrm{pH}$ (units) & 45 & 0.1 & 45 & 6.5 & 6.8 & 7.1 & 7.3 & 7.5 \\
\hline Temperature, water $\left({ }^{\circ} \mathrm{C}\right)$ & 45 & 0.5 & 45 & 20.5 & 21.5 & 22.3 & 23.2 & 27.0 \\
\hline Turbidity (NTU) & 45 & 0.1 & 45 & 0.2 & 0.4 & 0.8 & 1.4 & 33 \\
\hline Oxygen dissolved (mg/L) & 45 & 0.1 & 45 & 2.6 & 7.0 & 7.4 & 8.0 & 11 \\
\hline $\begin{array}{l}\text { Fecal coliform } 0.7 \mu \mathrm{m}-\mathrm{MF} \\
(\text { cols. } / 100 \mathrm{~mL})\end{array}$ & 17 & 0 & 17 & 37 & 205 & 440 & 1,900 & 9,600 \\
\hline $\begin{array}{l}\text { Solids, residue at } 105^{\circ} \mathrm{C}, \\
\text { suspended }(\mathrm{mg} / \mathrm{L})\end{array}$ & 18 & 1 & 13 & $<1$ & $\mathrm{e} 0.7$ & $\mathrm{e} 3.5$ & e6.8 & 46 \\
\hline $\begin{array}{l}\text { Nitrogen, nitrite plus } \\
\text { nitrate, dissolved (as N) }\end{array}$ & 13 & 0.05 & 13 & 0.13 & 0.22 & 0.27 & 0.33 & 0.50 \\
\hline $\begin{array}{l}\text { Nitrogen, ammonia } \\
\text { plus organic, total (as } \mathrm{N} \text { ) }\end{array}$ & 18 & 0.1 & 2 & $<0.1$ & -- & -- & -- & 0.40 \\
\hline Phosphorus, total (as P) & 18 & 0.05 & 9 & $<0.05$ & $\mathrm{e} 0.004$ & $\mathrm{e} 0.01$ & $\mathrm{e} 0.02$ & 0.09 \\
\hline
\end{tabular}


Table 19. Statistical summary of selected water-quality data collected at station 16270900 , Luluku Stream at alt $220 \mathrm{ft}$ near Kaneohe, Oahu, Hawaii, from February 1983 to April 1998

[inst., instantaneous; $\mu \mathrm{S} / \mathrm{cm}$ at $25^{\circ} \mathrm{C}$, microsiemens per centimeter at 25 degrees Celsius; $\mathrm{mg} / \mathrm{L}$, milligram per liter; $\mu \mathrm{g} / \mathrm{L}$, microgram per liter; <, less than; e, estimated using log-probability regression; $0.7 \mu \mathrm{m}-\mathrm{MF}, 0.7$ micron membrane filter; cols./100 mL, colonies per 100 milliliters; $\mathrm{ft}^{3} / \mathrm{s}$, cubic foot per second; ${ }^{\circ} \mathrm{C}$, degrees Celsius; NTU, nephelometric turbidity unit; --, no data or not computed; Range of dates are the time periods of water-quality data collection during the specified construction activity]

\begin{tabular}{|c|c|c|c|c|c|c|c|c|}
\hline \multirow[b]{2}{*}{ Property or constituent } & \multirow{2}{*}{$\begin{array}{l}\text { Number } \\
\text { of } \\
\text { samples }\end{array}$} & \multirow{2}{*}{$\begin{array}{l}\text { Minimum } \\
\text { detection } \\
\text { limit }\end{array}$} & \multirow{2}{*}{$\begin{array}{l}\text { Number } \\
\text { of } \\
\text { detections }\end{array}$} & \multirow{2}{*}{$\begin{array}{l}\text { Mini- } \\
\text { mum }\end{array}$} & \multicolumn{3}{|c|}{ Concentration percentile } & \multirow{2}{*}{$\begin{array}{l}\text { Maxi- } \\
\text { mum }\end{array}$} \\
\hline & & & & & 25 & 50 & 75 & \\
\hline \multicolumn{9}{|c|}{ Before construction, February 1983 to June 1989, physical, biological, and organic } \\
\hline Discharge, inst., $\left(\mathrm{ft}^{3} / \mathrm{s}\right)$ & 43 & 0 & 43 & 0.07 & 0.16 & 0.62 & 1.5 & 222 \\
\hline \multicolumn{9}{|l|}{ Specific conductance } \\
\hline$\left(\mu \mathrm{S} / \mathrm{cm}\right.$ at $\left.25^{\circ} \mathrm{C}\right)$ & 43 & 1 & 43 & 126 & 158 & 162 & 177 & 195 \\
\hline $\mathrm{pH}$ (units) & 43 & 0.1 & 43 & 6.3 & 6.9 & 7.1 & 7.4 & 8.3 \\
\hline Temperature, water $\left({ }^{\circ} \mathrm{C}\right)$ & 43 & 0.5 & 43 & 19.5 & 20.1 & 21.5 & 22.5 & 26.0 \\
\hline Turbidity (NTU) & 25 & 0.1 & 25 & 0.4 & 0.7 & 1.0 & 6.6 & 140 \\
\hline Oxygen dissolved (mg/L) & 25 & 0.1 & 25 & 6.8 & 7.6 & 8.2 & 8.8 & 9.8 \\
\hline $\begin{array}{l}\text { Fecal coliform } 0.7 \mu \mathrm{m}-\mathrm{MF} \\
(\text { cols. } / 100 \mathrm{~mL})\end{array}$ & 27 & 0 & 17 & 68 & 135 & 590 & 1,050 & 4,900 \\
\hline $\begin{array}{l}\text { Solids, residue at } 105^{\circ} \mathrm{C}, \\
\text { suspended }(\mathrm{mg} / \mathrm{L})\end{array}$ & 22 & 1 & 15 & $<1$ & $\mathrm{e} 1.2$ & $\mathrm{e} 5.5$ & e13 & 81 \\
\hline Carbon, organic, total $(\mathrm{mg} / \mathrm{L})$ & 8 & 0.1 & 8 & 0.6 & 0.6 & 0.8 & 4.2 & 5.4 \\
\hline Oil and grease, total $(\mathrm{mg} / \mathrm{L})$ & 8 & 1 & 1 & -- & -- & -- & -- & 1 \\
\hline \multicolumn{9}{|c|}{ During construction, June 1989 to December 1995, physical, biological, and organic } \\
\hline Discharge, inst., $\left(\mathrm{ft}^{3} / \mathrm{s}\right)$ & 68 & 0 & 68 & 0.20 & 0.94 & 1.2 & 1.7 & 93 \\
\hline Specific conductance & & & & & & & & \\
\hline$\left(\mu \mathrm{S} / \mathrm{cm}\right.$ at $\left.25^{\circ} \mathrm{C}\right)$ & 68 & 1 & 68 & 108 & 150 & 160 & 169 & 218 \\
\hline $\mathrm{pH}$ (units) & 68 & 0.1 & 68 & 6.0 & 7.2 & 7.5 & 7.7 & 8.1 \\
\hline Temperature, water $\left({ }^{\circ} \mathrm{C}\right)$ & 67 & 0.5 & 67 & 19.5 & 20.0 & 21.0 & 21.5 & 26.0 \\
\hline Turbidity (NTU) & 68 & 0.1 & 68 & 0.2 & 0.6 & 1.0 & 2.3 & 770 \\
\hline Oxygen dissolved (mg/L) & 68 & 0.1 & 68 & 6.9 & 8.2 & 8.4 & 8.8 & 9.5 \\
\hline $\begin{array}{l}\text { Fecal coliform } 0.7 \mu \mathrm{m}-\mathrm{MF} \\
(\text { cols. } / 100 \mathrm{~mL})\end{array}$ & 27 & 0 & 27 & 200 & 520 & 1,400 & 2,400 & 9,800 \\
\hline Solids, residue at $105^{\circ} \mathrm{C}$, & & & & & & & & \\
\hline suspended (mg/L) & 28 & 1 & 17 & $<1$ & $\mathrm{e} 0.14$ & $\mathrm{e} 1.5$ & e7.5 & 1,020 \\
\hline Carbon, organic, total $(\mathrm{mg} / \mathrm{L})$ & 14 & 0.1 & 14 & 0.40 & 0.70 & 0.90 & 1.6 & 9.9 \\
\hline Oil and grease, total $(\mathrm{mg} / \mathrm{L})$ & 14 & 1 & 0 & -- & -- & -- & -- & -- \\
\hline \multicolumn{9}{|c|}{ After construction, January 1996 to April 1998, physical, biological, and organic } \\
\hline Discharge, inst., $\left(\mathrm{ft}^{3} / \mathrm{s}\right)$ & 18 & 0 & 18 & 0.74 & 1.1 & 1.3 & 1.4 & 10 \\
\hline Specific conductance & & & & & & & & \\
\hline$\left(\mu \mathrm{S} / \mathrm{cm}\right.$ at $\left.25^{\circ} \mathrm{C}\right)$ & 21 & 1 & 21 & 120 & 156 & 158 & 172 & 187 \\
\hline $\mathrm{pH}$ (units) & 21 & 0.1 & 21 & 7.0 & 7.4 & 7.6 & 7.7 & 7.9 \\
\hline Temperature, water $\left({ }^{\circ} \mathrm{C}\right)$ & 18 & 0.5 & 18 & 18.5 & 20.0 & 21.0 & 21.5 & 22.0 \\
\hline Turbidity (NTU) & 20 & 0.1 & 20 & 0.10 & 0.30 & 0.34 & 0.50 & 34 \\
\hline Oxygen dissolved (mg/L) & 20 & 0.1 & 20 & 6.9 & 8.5 & 8.8 & 8.8 & 9.2 \\
\hline $\begin{array}{l}\text { Fecal coliform } 0.7 \mu \mathrm{m}-\mathrm{MF} \\
\text { (cols. } / 100 \mathrm{~mL})\end{array}$ & 9 & 0 & 9 & 130 & 175 & 230 & 410 & 1,000 \\
\hline $\begin{array}{l}\text { Solids, residue at } 105^{\circ} \mathrm{C}, \\
\text { suspended }(\mathrm{mg} / \mathrm{L})\end{array}$ & 9 & 1 & 7 & $<1$ & $\mathrm{e} 0.8$ & $\mathrm{e} 1.0$ & $\mathrm{e} 3.0$ & 3.0 \\
\hline Carbon, organic, total $(\mathrm{mg} / \mathrm{L})$ & 4 & 0.1 & 4 & 0.5 & -- & 2.0 & -- & 3.6 \\
\hline Oil and grease, total $(\mathrm{mg} / \mathrm{L})$ & 4 & 1 & 0 & - & -- & -- & -- & -- \\
\hline
\end{tabular}


Table 19. Statistical summary of selected water-quality data collected at station 16270900 , Luluku Stream at alt $220 \mathrm{ft}$ near Kaneohe, Oahu, Hawaii, from February 1983 to April 1998--Continued

\begin{tabular}{|c|c|c|c|c|c|c|c|c|}
\hline \multirow[b]{2}{*}{ Property or constituent } & \multirow{2}{*}{$\begin{array}{l}\text { Number } \\
\text { of } \\
\text { samples }\end{array}$} & \multirow{2}{*}{$\begin{array}{l}\text { Minimum } \\
\text { detection } \\
\text { limit }\end{array}$} & \multirow{2}{*}{$\begin{array}{l}\text { Number } \\
\text { of } \\
\text { detections }\end{array}$} & \multirow{2}{*}{$\begin{array}{l}\text { Mini- } \\
\text { mum }\end{array}$} & \multicolumn{3}{|c|}{ Concentration percentile } & \multirow{2}{*}{$\begin{array}{l}\text { Maxi- } \\
\text { mum }\end{array}$} \\
\hline & & & & & 25 & 50 & 75 & \\
\hline \multicolumn{9}{|c|}{ Before construction, February 1983 to June 1989, major ions and nutrients (mg/L) } \\
\hline Hardness, total $\left(\right.$ as $\left.\mathrm{CaCO}_{3}\right)$ & ) 8 & 1 & 8 & 46 & 46 & 47 & 52 & 61 \\
\hline Calcium, dissolved & 8 & 0.02 & 8 & 8.5 & 8.8 & 9.1 & 10 & 12 \\
\hline Magnesium, dissolved & 8 & 0.01 & 8 & 5.7 & 5.8 & 6.0 & 6.8 & 7.5 \\
\hline Sodium, dissolved & 8 & 0.2 & 8 & 10 & 12 & 13 & 14 & 14 \\
\hline Potassium, dissolved & 8 & 0.1 & 8 & 0.9 & 1.0 & 1.2 & 2.0 & 3.1 \\
\hline Alkalinity $\left(\right.$ as $\left.\mathrm{CaCO}_{3}\right)$ & 7 & 1 & 7 & 41 & 44 & 47 & 51 & 55 \\
\hline Sulfate, dissolved & 8 & 0.1 & 7 & 3.5 & e3.7 & e4.8 & e7.2 & 15 \\
\hline Chloride, dissolved & 8 & 0.1 & 8 & 15 & 15 & 16 & 18 & 20 \\
\hline Fluoride, dissolved & 8 & 0.1 & 3 & 0.1 & -- & 0.2 & -- & 0.3 \\
\hline Silica, dissolved & 8 & 0.01 & 8 & 21 & 23 & 27 & 28 & 29 \\
\hline $\begin{array}{l}\text { Nitrogen, nitrite plus } \\
\text { nitrate, total (as N) }\end{array}$ & 19 & 0.05 & 13 & $<0.2$ & $\mathrm{e} 0.10$ & $\mathrm{e} 0.20$ & $\mathrm{e} 0.40$ & 1.3 \\
\hline $\begin{array}{l}\text { Nitrogen, ammonia } \\
\text { plus organic, total (as N) }\end{array}$ & 19 & 0.2 & 12 & $<0.2$ & $\mathrm{e} 0.10$ & $\mathrm{e} 0.20$ & $\mathrm{e} 0.50$ & 1.0 \\
\hline Phosphorus, total (as P) & 19 & 0.01 & 18 & 0.01 & $\mathrm{e} 0.01$ & $\mathrm{e} 0.02$ & $\mathrm{e} 0.03$ & 0.29 \\
\hline \multicolumn{9}{|c|}{ During construction, June 1989 to December 1995, major ions and nutrients (mg/L) } \\
\hline Hardness, total (as $\left.\mathrm{CaCO}_{3}\right)$ & ) 14 & 1 & 14 & 21 & 44 & 46 & 50 & 60 \\
\hline Calcium, dissolved & 14 & 0.02 & 14 & 4.5 & 8.8 & 9.3 & 10 & 12 \\
\hline Magnesium, dissolved & 14 & 0.01 & 14 & 2.4 & 5.3 & 5.5 & 5.8 & 7.2 \\
\hline Sodium, dissolved & 14 & 0.2 & 14 & 6.5 & 13 & 13 & 13 & 14 \\
\hline Potassium, dissolved & 14 & 0.1 & 14 & 0.8 & 0.9 & 1.0 & 1.3 & 3.0 \\
\hline Alkalinity (as $\mathrm{CaCO}_{3}$ ) & 14 & 1 & 14 & 22 & 47 & 49 & 50 & 61 \\
\hline Sulfate, dissolved & 14 & 0.1 & 14 & 1.9 & 2.9 & 3.6 & 4.6 & 10 \\
\hline Chloride, dissolved & 14 & 0.1 & 14 & 9.0 & 15 & 16 & 17 & 19 \\
\hline Fluoride, dissolved & 14 & 0.1 & 3 & $<0.1$ & -- & -- & -- & 0.2 \\
\hline Silica, dissolved & 14 & 0.01 & 14 & 10 & 26 & 28 & 29 & 30 \\
\hline $\begin{array}{l}\text { Nitrogen, nitrite plus } \\
\text { nitrate, total (as N) }\end{array}$ & 16 & 0.05 & 15 & $<0.05$ & $\mathrm{e} 0.10$ & $\mathrm{e} 0.15$ & $\mathrm{e} 0.20$ & 0.40 \\
\hline $\begin{array}{l}\text { Nitrogen, nitrite plus } \\
\text { nitrate, dissolved (as N) }\end{array}$ & 8 & 0.05 & 8 & 0.10 & 0.15 & 0.17 & 0.19 & 0.37 \\
\hline $\begin{array}{l}\text { Nitrogen, ammonia } \\
\text { plus organic, total (as N) }\end{array}$ & 28 & 0.2 & 9 & $<0.20$ & $\mathrm{e} 0.07$ & $\mathrm{e} 0.12$ & $\mathrm{e} 0.28$ & 0.80 \\
\hline Phosphorus, total (as P) & 28 & 0.01 & 25 & $<0.01$ & $\mathrm{e} 0.02$ & $\mathrm{e} 0.03$ & $\mathrm{e} 0.03$ & 1.0 \\
\hline \multicolumn{9}{|c|}{ After construction, January 1996 to April 1998, major ions and nutrients (mg/L) } \\
\hline Hardness, total (as $\left.\mathrm{CaCO}_{3}\right)$ & 4 & 1 & 4 & 45 & -- & 49 & -- & 53 \\
\hline Calcium, dissolved & 4 & 0.02 & 4 & 9.0 & -- & 10 & -- & 11 \\
\hline Magnesium, dissolved & 4 & 0.01 & 4 & 5.5 & -- & 5.8 & -- & 6.1 \\
\hline Sodium, dissolved & 4 & 0.2 & 4 & 11 & -- & 12 & -- & 12 \\
\hline Potassium, dissolved & 4 & 0.1 & 4 & 0.88 & -- & 0.94 & -- & 1.0 \\
\hline Alkalinity $\left(\right.$ as $\left.\mathrm{CaCO}_{3}\right)$ & 4 & 1 & 4 & 48 & -- & 51 & -- & 54 \\
\hline Sulfate, dissolved & 4 & 0.1 & 4 & 4.0 & -- & 5.4 & -- & 6.8 \\
\hline Chloride, dissolved & 4 & 0.1 & 4 & 16 & -- & 16 & -- & 17 \\
\hline Fluoride, dissolved & 4 & 0.1 & 0 & -- & -- & -- & -- & -- \\
\hline Silica, dissolved & 4 & 0.01 & 4 & 25 & -- & 26 & -- & 27 \\
\hline $\begin{array}{l}\text { Nitrogen, nitrite plus } \\
\text { nitrate, dissolved (as N) }\end{array}$ & 9 & 0.05 & 9 & 0.08 & 0.11 & 0.17 & 0.20 & 0.23 \\
\hline $\begin{array}{l}\text { Nitrogen, ammonia } \\
\text { plus organic, total (as } \mathrm{N} \text { ) }\end{array}$ & 9 & 0.1 & 0 & -- & -- & -- & -- & -- \\
\hline Phosphorus, total (as P) & 9 & 0.05 & 7 & $<0.05$ & $\mathrm{e} 0.01$ & $\mathrm{e} 0.01$ & $\mathrm{e} 0.02$ & 0.03 \\
\hline
\end{tabular}


Table 19. Statistical summary of selected water-quality data collected at station 16270900 , Luluku Stream at alt $220 \mathrm{ft}$ near Kaneohe, Oahu, Hawaii, from February 1983 to April 1998--Continued

\begin{tabular}{|c|c|c|c|c|c|c|c|c|}
\hline \multirow[b]{2}{*}{ Property or constituent } & \multirow{2}{*}{$\begin{array}{c}\text { Number } \\
\text { of } \\
\text { samples }\end{array}$} & \multirow{2}{*}{$\begin{array}{l}\text { Minimum } \\
\text { detection } \\
\text { limit }\end{array}$} & \multirow{2}{*}{$\begin{array}{l}\text { Number } \\
\text { of } \\
\text { detections }\end{array}$} & \multirow{2}{*}{$\begin{array}{l}\text { Mini- } \\
\text { mum }\end{array}$} & \multicolumn{3}{|c|}{ Concentration percentile } & \multirow{2}{*}{$\begin{array}{l}\text { Maxi- } \\
\text { mum }\end{array}$} \\
\hline & & & & & 25 & 50 & 75 & \\
\hline \multicolumn{9}{|c|}{ Before construction, February 1983 to June 1989 , trace metals $(\mu \mathrm{g} / \mathrm{L})$} \\
\hline Aluminum, total recoverable & e 8 & 10 & 8 & 20 & 52 & 80 & 252 & 1,600 \\
\hline Aluminum, dissolved & 8 & 10 & 5 & 10 & $\mathrm{e} 1.5$ & e10 & e28 & 160 \\
\hline Cadmium, total recoverable & 8 & 1 & 1 & -- & -- & -- & -- & 1.0 \\
\hline Cadmium, dissolved & 8 & 1 & 1 & -- & -- & -- & -- & 2.0 \\
\hline Chromium, total recoverable & 8 & 10 & 4 & $<1$ & -- & -- & -- & 20 \\
\hline Chromium, dissolved & 8 & 1 & 3 & $<1$ & -- & -- & -- & 7.0 \\
\hline Copper, total recoverable & 8 & 1 & 7 & $<1$ & $\mathrm{e} 2.2$ & $\mathrm{e} 3.5$ & $\mathrm{e} 7.2$ & 8.0 \\
\hline Copper, dissolved & 8 & 1 & 5 & $<1$ & $\mathrm{e} 0.72$ & $\mathrm{e} 1.5$ & $\mathrm{e} 2.8$ & 3.0 \\
\hline Iron, total recoverable & 8 & 10 & 8 & 60 & 90 & 125 & 1,325 & 4,400 \\
\hline Iron, dissolved & 8 & 3 & 8 & 20 & 20 & 20 & 65 & 100 \\
\hline Lead, total recoverable & 8 & 1 & 4 & $<1$ & -- & -- & -- & 10 \\
\hline Lead, dissolved & 8 & 1 & 3 & $<1$ & -- & -- & -- & 2.0 \\
\hline Nickel, total recoverable & 8 & 1 & 8 & 1.0 & 2.2 & 5.5 & 8.0 & 9.0 \\
\hline Nickel, dissolved & 8 & 1 & 3 & $<1$ & -- & -- & -- & 5.0 \\
\hline Zinc, total recoverable & 8 & 10 & 6 & $<10$ & 4.4 & e10 & 20 & 140 \\
\hline Zinc, dissolved & 8 & 3 & 5 & $<3.0$ & 1.4 & $\mathrm{e} 4.5$ & 8.8 & 19 \\
\hline \multicolumn{9}{|c|}{ During construction, June 1989 to December 1995 , trace metals $(\mu \mathrm{g} / \mathrm{L})$} \\
\hline Aluminum, total recoverable & e 14 & 10 & 14 & 20 & 62 & 120 & 6,300 & 6,300 \\
\hline Aluminum, dissolved & 14 & 10 & 9 & $<10$ & $\mathrm{e} 2.8$ & e10 & e30 & 170 \\
\hline Cadmium, total recoverable & 14 & 1 & 0 & -- & -- & -- & -- & -- \\
\hline Cadmium, dissolved & 5 & 1 & 0 & -- & -- & -- & -- & -- \\
\hline Chromium, total recoverable & e 14 & 1 & 10 & $<10$ & $\mathrm{e} 1.0$ & $\mathrm{e} 1.5$ & e2.0 & 30 \\
\hline Chromium, dissolved & 5 & 1 & 2 & $<1$ & -- & -- & -- & 3.0 \\
\hline Copper, total recoverable & 14 & 1 & 12 & $<1$ & $\mathrm{e} 0.22$ & e1.0 & $\mathrm{e} 2.2$ & 10 \\
\hline Copper, dissolved & 5 & 1 & 3 & $<1$ & -- & -- & -- & 3.0 \\
\hline Iron, total recoverable & 14 & 10 & 14 & 40 & 108 & 220 & 400 & 8,600 \\
\hline Iron, dissolved & 14 & 3 & 14 & 10 & 20 & 25 & 38 & 230 \\
\hline Lead, total recoverable & 14 & 1 & 4 & $<1$ & -- & -- & -- & 6 \\
\hline Lead, dissolved & 5 & 1 & 1 & -- & -- & -- & -- & 1.0 \\
\hline Nickel, total recoverable & 14 & 1 & 6 & $<1$ & $\mathrm{e} 0.06$ & $\mathrm{e} 0.27$ & e13 & 13 \\
\hline Nickel, dissolved & 14 & 1 & 1 & -- & -- & -- & -- & 1.0 \\
\hline Zinc, total recoverable & 14 & 10 & 4 & $<10$ & -- & -- & -- & 30 \\
\hline Zinc, dissolved & 5 & 3 & 4 & $<3$ & -- & -- & -- & 6 \\
\hline \multicolumn{9}{|c|}{ After construction, January 1996 to April 1998, trace metals $(\mu \mathrm{g} / \mathrm{L})$} \\
\hline Aluminum, total recoverable & e 4 & 10 & 4 & 10 & -- & 30 & -- & 50 \\
\hline Aluminum, dissolved & 4 & 10 & 0 & -- & -- & -- & -- & -- \\
\hline Cadmium, total recoverable & 4 & 1 & 0 & -- & -- & -- & -- & -- \\
\hline Cadmium, dissolved & 0 & 1 & -- & -- & -- & -- & -- & -- \\
\hline Chromium, total recoverable & 4 & 1 & 2 & $<1$ & -- & -- & -- & 1 \\
\hline Chromium, dissolved & 0 & 1 & -- & -- & -- & -- & -- & -- \\
\hline Copper, total recoverable & 4 & 1 & 1 & -- & -- & -- & -- & 1 \\
\hline Copper, dissolved & 0 & 1 & -- & -- & -- & -- & -- & -- \\
\hline Iron, total recoverable & 4 & 10 & 4 & 40 & -- & -- & -- & 100 \\
\hline Iron, dissolved & 4 & 3 & 3 & 5.0 & -- & -- & -- & 6.0 \\
\hline Lead, total recoverable & 4 & 1 & 0 & -- & -- & -- & -- & -- \\
\hline Lead, dissolved & 0 & 1 & -- & -- & -- & -- & -- & -- \\
\hline Nickel, total recoverable & 4 & 1 & 0 & -- & -- & -- & -- & -- \\
\hline Nickel, dissolved & 4 & 1 & 0 & -- & -- & -- & -- & -- \\
\hline Zinc, total recoverable & 4 & 10 & 0 & -- & -- & -- & -- & -- \\
\hline Zinc, dissolved & 0 & 20 & -- & -- & -- & -- & -- & -- \\
\hline
\end{tabular}


Table 20. Statistical summary of selected water-quality data collected at station 16272200 , Kamooalii Stream below Luluku Stream near Kaneohe, Oahu, Hawaii, from February 1983 to April 1998

[inst., instantaneous; $\mu \mathrm{S} / \mathrm{cm}$ at $25^{\circ} \mathrm{C}$, microsiemens per centimeter at 25 degrees Celsius; $\mathrm{mg} / \mathrm{L}$, milligram per liter; $\mu \mathrm{g} / \mathrm{L}$, microgram per liter; <, less than; e, estimated using log-probability regression; $0.7 \mu \mathrm{m}-\mathrm{MF}, 0.7$ micron membrane filter; cols. $/ 100 \mathrm{~mL}$, colonies per 100 milliliters; $\mathrm{ft}^{3} / \mathrm{s}$, cubic foot per second; ${ }^{\circ} \mathrm{C}$, degrees Celsius; $\mathrm{NTU}$, nephelometric turbidity unit; --, no data or not computed; Range of dates are the time periods of water-quality data collection during the specified construction activity]

\begin{tabular}{|c|c|c|c|c|c|c|c|c|}
\hline Property or constituent & $\begin{array}{l}\text { Number } \\
\text { of } \\
\text { samples }\end{array}$ & $\begin{array}{l}\text { Minimum } \\
\text { detection } \\
\text { limit }\end{array}$ & $\begin{array}{l}\text { Number } \\
\text { of } \\
\text { detections }\end{array}$ & $\begin{array}{l}\text { Mini- } \\
\text { mum }\end{array}$ & \multicolumn{3}{|c|}{ Concentration percentile } & $\begin{array}{l}\text { Maxi- } \\
\text { mum }\end{array}$ \\
\hline \multicolumn{9}{|c|}{ Before construction, February 1983, physical, biological, and organic } \\
\hline Discharge, inst., $\left(\mathrm{ft}^{3} / \mathrm{s}\right)$ & 1 & 0 & 1 & -- & -- & -- & -- & 14 \\
\hline $\begin{array}{l}\text { Specific conductance } \\
\left(\mathrm{uS} / \mathrm{cm} \text { at } 25^{\circ} \mathrm{C}\right)\end{array}$ & 1 & 1 & 1 & -- & -- & & & \\
\hline $\mathrm{pH}$ (units) & 1 & 0.1 & 1 & - & -- & $\begin{array}{l}-- \\
--\end{array}$ & -- & 7.1 \\
\hline Temperature, water $\left({ }^{\circ} \mathrm{C}\right)$ & 1 & 0.5 & 1 & -- & -- & -- & -- & 20.5 \\
\hline Turbidity (NTU) & 1 & 0.1 & 1 & -- & -- & -- & -- & 1.5 \\
\hline Oxygen dissolved (mg/L) & 1 & 0.1 & 1 & -- & -- & -- & -- & 8.6 \\
\hline $\begin{array}{l}\text { Fecal coliform } 0.7 \mu \mathrm{m}-\mathrm{MF} \\
(\text { cols. } / 100 \mathrm{~mL})\end{array}$ & 1 & 0 & -- & -- & -- & -- & -- & 19 \\
\hline $\begin{array}{l}\text { Solids, residue at } 105^{\circ} \mathrm{C}, \\
\text { suspended }(\mathrm{mg} / \mathrm{L})\end{array}$ & 1 & 1 & 0 & -- & -- & -- & -- & -- \\
\hline Carbon, organic, total (mg/L) & 1 & 0.1 & 1 & -- & -- & -- & -- & 1.8 \\
\hline Oil and grease, total (mg/L) & 1 & 1 & 0 & -- & -- & -- & -- & -- \\
\hline \multicolumn{9}{|c|}{ During construction, February 1983 to December 1995, physical, biological, and organic } \\
\hline Discharge, inst., $\left(\mathrm{ft}^{3} / \mathrm{s}\right)$ & 159 & 0 & 159 & 0.29 & 5.7 & 9.4 & 180 & 1180 \\
\hline $\begin{array}{l}\text { Specific conductance } \\
\left(\mu \mathrm{S} / \mathrm{cm} \text { at } 25^{\circ} \mathrm{C}\right)\end{array}$ & 130 & 1 & 130 & 80 & 170 & 180 & 190 & 220 \\
\hline $\mathrm{pH}$ (units) & 118 & 0.1 & 118 & 6.4 & 7.3 & 7.8 & 8.0 & 8.5 \\
\hline Temperature, water $\left({ }^{\circ} \mathrm{C}\right)$ & 118 & 0.5 & 118 & 20.5 & 23.0 & 24.8 & 26.2 & 31.0 \\
\hline Turbidity (NTU) & 141 & 0.1 & 141 & 0.5 & 1.6 & 2.6 & 85 & 550 \\
\hline Oxygen dissolved (mg/L) & 100 & 0.1 & 100 & 5.4 & 8.0 & 8.4 & 8.8 & 10.8 \\
\hline $\begin{array}{l}\text { Fecal coliform } 0.7 \mu \mathrm{m}-\mathrm{MF} \\
(\text { cols. } / 100 \mathrm{~mL})\end{array}$ & 46 & 0 & 46 & 16 & 73 & 135 & 292 & 22,000 \\
\hline $\begin{array}{l}\text { Solids, residue at } 105^{\circ} \mathrm{C}, \\
\text { suspended }(\mathrm{mg} / \mathrm{L})\end{array}$ & 81 & 1 & 76 & $<1$ & e5.0 & e9.0 & e186 & 752 \\
\hline Carbon, organic, total (mg/L) & 36 & 0.1 & 36 & 1.0 & 1.6 & 2.0 & 7.9 & 27 \\
\hline Oil and grease, total $(\mathrm{mg} / \mathrm{L})$ & 25 & 1 & 2 & $<1$ & -- & -- & -- & 2 \\
\hline \multicolumn{9}{|c|}{ After construction, January 1996 to April 1998, physcal, biological, and organic } \\
\hline Discharge, inst., $\left(\mathrm{ft}^{3} / \mathrm{s}\right)$ & 18 & 0 & 18 & 4.8 & 7.1 & 9.7 & 13.2 & 19 \\
\hline $\begin{array}{l}\text { Specific conductance } \\
\left(\mu \mathrm{S} / \mathrm{cm} \text { at } 25^{\circ} \mathrm{C}\right)\end{array}$ & 17 & 1 & 17 & 138 & 180 & 190 & 196 & 215 \\
\hline $\mathrm{pH}$ (units) & 18 & 0.1 & 18 & 6.5 & 7.6 & 7.9 & 8.0 & 8.1 \\
\hline Temperature, water $\left({ }^{\circ} \mathrm{C}\right)$ & 18 & 0.5 & 18 & 19.5 & 22.5 & 23.8 & 25.5 & 27.0 \\
\hline Turbidity (NTU) & 18 & 0.1 & 18 & 0.3 & 0.8 & 1.4 & 2.1 & 6.0 \\
\hline Oxygen dissolved (mg/L) & 20 & 0.1 & 20 & 7.9 & 8.4 & 8.8 & 9.1 & 9.5 \\
\hline $\begin{array}{l}\text { Fecal coliform } 0.7 \mu \mathrm{m}-\mathrm{MF} \\
(\text { cols. } / 100 \mathrm{~mL})\end{array}$ & 8 & 0 & 8 & 50 & 83 & 105 & 155 & 270 \\
\hline $\begin{array}{l}\text { Solids, residue at } 105^{\circ} \mathrm{C}, \\
\text { suspended }(\mathrm{mg} / \mathrm{L})\end{array}$ & 9 & 1 & 5 & $<1$ & $\mathrm{e} 0.2$ & e1.0 & $\mathrm{e} 4.0$ & $\mathrm{e} 8.0$ \\
\hline Carbon, organic, total $(\mathrm{mg} / \mathrm{L})$ & 4 & 0.1 & 4 & 1.1 & -- & 1.4 & -- & 2.1 \\
\hline Oil and grease, total (mg/L) & 4 & 1 & 0 & -- & -- & -- & -- & -- \\
\hline
\end{tabular}


Table 20. Statistical summary of selected water-quality data collected at station 16272200 , Kamooalii Stream below Luluku Stream near Kaneohe, Oahu, Hawaii, from February 1983 to April 1998--Continued

\begin{tabular}{|c|c|c|c|c|c|c|c|c|}
\hline \multirow[b]{2}{*}{ Property or constituent } & \multirow{2}{*}{$\begin{array}{l}\text { Number } \\
\text { of } \\
\text { samples }\end{array}$} & \multirow{2}{*}{$\begin{array}{l}\text { Minimum } \\
\text { detection } \\
\text { limit }\end{array}$} & \multirow{2}{*}{$\begin{array}{l}\text { Number } \\
\text { of } \\
\text { detections }\end{array}$} & \multirow{2}{*}{$\begin{array}{l}\text { Mini- } \\
\text { mum }\end{array}$} & \multicolumn{3}{|c|}{ Concentration percentile } & \multirow{2}{*}{$\begin{array}{l}\text { Maxi } \\
\text { mum }\end{array}$} \\
\hline & & & & & 25 & 50 & 75 & \\
\hline \multicolumn{9}{|c|}{ Before construction, February 1983, major ions and nutrients $(\mathrm{mg} / \mathrm{L})$} \\
\hline Hardness, total (as $\left.\mathrm{CaCO}_{3}\right)$ & 1 & 1 & 1 & -- & -- & -- & -- & 52 \\
\hline Calcium, dissolved & 1 & 0.02 & 1 & -- & -- & -- & -- & 8.2 \\
\hline Magnesium, dissolved & 1 & 0.01 & 1 & -- & -- & -- & -- & 7.6 \\
\hline Sodium, dissolved & 1 & 0.2 & 1 & -- & -- & -- & -- & 15 \\
\hline Potassium, dissolved & 1 & 0.1 & 1 & -- & -- & -- & -- & 0.9 \\
\hline Alkalinity (as $\mathrm{CaCO}_{3}$ ) & 1 & 1 & 1 & -- & -- & -- & -- & 59 \\
\hline Sulfate, dissolved & 1 & 0.1 & 1 & -- & -- & -- & -- & 6.8 \\
\hline Chloride, dissolved & 1 & 0.1 & 1 & -- & -- & -- & -- & 18 \\
\hline Fluoride, dissolved & 1 & 0.1 & 1 & -- & -- & -- & -- & -- \\
\hline Silica, dissolved & 1 & 0.01 & 1 & -- & -- & -- & -- & 22 \\
\hline \multicolumn{9}{|l|}{ Nitrogen, nitrite plus } \\
\hline \multicolumn{9}{|l|}{ Nitrogen, ammonia } \\
\hline plus organic, total (as N) & 1 & 0.2 & 1 & -- & -- & -- & -- & 0.80 \\
\hline Phosphorus, total (as P) & 1 & 0.01 & 1 & -- & -- & -- & -- & 0.05 \\
\hline \multicolumn{9}{|c|}{ During construction, Febrary 1983 to December 1995, major ions and nutrients (mg/L) } \\
\hline Hardness, total $\left(\right.$ as $\left.\mathrm{CaCO}_{3}\right)$ & 26 & 1 & 26 & 26 & 51 & 54 & 56 & 67 \\
\hline Calcium, dissolved & 26 & 0.02 & 26 & 5.0 & 7.8 & 8.9 & 9.3 & 11 \\
\hline Magnesium, dissolved & 26 & 0.01 & 26 & 3.3 & 7.1 & 7.8 & 8.3 & 9.6 \\
\hline Sodium, dissolved & 26 & 0.2 & 26 & 5.7 & 13 & 15 & 15 & 17 \\
\hline Potassium, dissolved & 26 & 0.1 & 26 & 0.9 & 0.9 & 1.1 & 1.3 & 2.1 \\
\hline Alkalinity (as $\mathrm{CaCO}_{3}$ ) & 26 & 1 & 26 & 21 & 46 & 54 & 55 & 61 \\
\hline Sulfate, dissolved & 26 & 0.1 & 26 & 3.6 & 6.8 & 7.8 & 9.3 & 25 \\
\hline Chloride, dissolved & 26 & 0.1 & 26 & 6.6 & 17 & 18 & 19 & 22 \\
\hline Fluoride, dissolved & 26 & 0.1 & 8 & $<0.1$ & $\mathrm{e} 0.02$ & $\mathrm{e} 0.05$ & $\mathrm{e} 0.10$ & 0.30 \\
\hline Silica, dissolved & 26 & 0.01 & 26 & 9.0 & 20 & 21 & 22 & 23 \\
\hline $\begin{array}{l}\text { Nitrogen, nitrite plus } \\
\text { nitrate, total (as N) }\end{array}$ & 54 & 0.1 & 51 & $<0.1$ & $\mathrm{e} 0.20$ & $\mathrm{e} 0.30$ & $\mathrm{e} 0.30$ & 0.80 \\
\hline $\begin{array}{l}\text { Nitrogen, nitrite plus } \\
\text { nitrate, dissolved (as N) }\end{array}$ & 7 & 0.05 & 7 & 0.22 & 0.25 & 0.29 & 0.31 & 0.41 \\
\hline Nitrogen, ammonia & & & & & & & & \\
\hline plus organic, total (as N) & 66 & 0.2 & 45 & $<0.2$ & $\mathrm{e} 0.14$ & $\mathrm{e} 0.30$ & $\mathrm{e} 0.82$ & 4.6 \\
\hline Phosphorus, total (as P) & 66 & 0.01 & 60 & $<0.01$ & $\mathrm{e} 0.01$ & $\mathrm{e} 0.02$ & $\mathrm{e} 0.26$ & 0.75 \\
\hline \multicolumn{9}{|c|}{ After construction, January 1996 to April 1998, major ions and nutrients (mg/L) } \\
\hline Hardness, total (as $\left.\mathrm{CaCO}_{3}\right)$ & 4 & 1 & 4 & 55 & -- & 58 & -- & 60 \\
\hline Calcium, dissolved & 4 & 0.02 & 4 & 9.4 & -- & 9.7 & -- & 10 \\
\hline Magnesium, dissolved & 4 & 0.01 & 4 & 7.4 & -- & 8.0 & -- & 8.6 \\
\hline Sodium, dissolved & 4 & 0.2 & 4 & 13 & -- & 14 & -- & 14 \\
\hline Potassium, dissolved & 4 & 0.1 & 4 & 0.9 & -- & 1.2 & -- & 1.5 \\
\hline Alkalinity $\left(\right.$ as $\mathrm{CaCO}_{3}$ ) & 4 & 1 & 4 & 55 & -- & 57 & -- & 59 \\
\hline Sulfate, dissolved & 4 & 0.1 & 4 & 6.9 & -- & 7.8 & -- & 8.8 \\
\hline Chloride, dissolved & 4 & 0.1 & 4 & 17 & -- & 18 & -- & 20 \\
\hline Fluoride, dissolved & 4 & 0.1 & 0 & -- & -- & -- & -- & -- \\
\hline Silica, dissolved & 4 & 0.01 & 4 & 18 & -- & 19 & -- & 20 \\
\hline $\begin{array}{l}\text { Nitrogen, nitrite plus } \\
\text { nitrate, dissolved (as N) }\end{array}$ & 9 & 0.05 & 9 & 0.28 & 0.32 & 0.33 & 0.36 & 0.41 \\
\hline $\begin{array}{l}\text { Nitrogen, ammonia } \\
\text { plus organic, total (as } \mathrm{N} \text { ) }\end{array}$ & 9 & 0.1 & 4 & $<0.1$ & -- & -- & -- & 0.20 \\
\hline Phosphorus, total (as P) & 9 & 0.05 & 8 & $<0.05$ & $\mathrm{e} 0.01$ & $\mathrm{e} 0.02$ & $\mathrm{e} 0.02$ & 0.03 \\
\hline
\end{tabular}


Table 20. Statistical summary of selected water-quality data collected at station 272200, Kamooalii Stream below Luluku Stream near Kaneohe, Oahu, Hawaii, from February 1983 to April 1998--Continued

\begin{tabular}{|c|c|c|c|c|c|c|c|c|}
\hline \multirow[b]{2}{*}{ Property or constituent } & \multirow{2}{*}{$\begin{array}{l}\text { Number } \\
\text { of } \\
\text { samples }\end{array}$} & \multirow{2}{*}{$\begin{array}{l}\text { Minimum } \\
\text { detection } \\
\text { limit }\end{array}$} & \multirow{2}{*}{$\begin{array}{l}\text { Number } \\
\text { of } \\
\text { detections }\end{array}$} & \multirow{2}{*}{$\begin{array}{l}\text { Mini- } \\
\text { mum }\end{array}$} & \multicolumn{3}{|c|}{ Concentration percentile } & \multirow{2}{*}{$\begin{array}{l}\text { Maxi } \\
\text { mum }\end{array}$} \\
\hline & & & & & 25 & 50 & 75 & \\
\hline \multicolumn{9}{|c|}{ Before construction, February 1983, trace metals $(\mu \mathrm{g} / \mathrm{L})$} \\
\hline Aluminum, total recoverable & e 1 & 10 & 1 & -- & -- & -- & -- & 170 \\
\hline Aluminum, dissolved & 1 & 10 & 1 & -- & -- & -- & -- & 10 \\
\hline Cadmium, total recoverable & 1 & 1 & 0 & -- & -- & -- & -- & -- \\
\hline Cadmium, dissolved & 1 & 1 & 0 & -- & -- & -- & -- & -- \\
\hline Chromium, total recoverable & 1 & 10 & 0 & -- & -- & -- & -- & -- \\
\hline Chromium, dissolved & 1 & 1 & 0 & -- & -- & -- & & -- \\
\hline Copper, total recoverable & 1 & 1 & 0 & -- & -- & -- & -- & -- \\
\hline Copper, dissolved & 1 & 1 & 0 & -- & -- & -- & -- & -- \\
\hline Iron, total recoverable & 0 & 10 & -- & -- & -- & -- & -- & -- \\
\hline Iron, dissolved & 1 & 3 & 0 & -- & -- & -- & -- & -- \\
\hline Lead, total recoverable & 1 & 1 & 0 & -- & -- & -- & -- & -- \\
\hline Lead, dissolved & 1 & 1 & 0 & -- & -- & -- & -- & -- \\
\hline Nickel, total recoverable & 1 & 1 & 1 & -- & -- & -- & -- & 5.0 \\
\hline Nickel, dissolved & 1 & 1 & 1 & -- & -- & -- & -- & 1.0 \\
\hline Zinc, total recoverable & 1 & 10 & 1 & -- & -- & -- & -- & 10 \\
\hline Zinc, dissolved & 1 & 3 & 1 & -- & -- & -- & -- & 4.0 \\
\hline \multicolumn{9}{|c|}{ During construction, February 1983 to December 1995, trace metals $(\mu \mathrm{g} / \mathrm{L})$} \\
\hline Aluminum, total recoverable & e 28 & 10 & 28 & 90 & 140 & 245 & 460 & 34,000 \\
\hline Aluminum, dissolved & 26 & 10 & 21 & $<10$ & $\mathrm{e} 8.8$ & e20 & e32 & 1,200 \\
\hline Cadmium, total recoverable & 28 & 1 & 4 & $<1$ & -- & -- & -- & 1 \\
\hline Cadmium, dissolved & 17 & 1 & 1 & $<1$ & -- & -- & -- & 1 \\
\hline Chromium, total recoverable & e 28 & 1 & 17 & $<1$ & $\mathrm{e} 0.35$ & e1.8 & e8.3 & 80 \\
\hline Chromium, dissolved & 17 & 1 & 1 & -- & -- & -- & -- & 3.0 \\
\hline Copper, total recoverable & 28 & 1 & 27 & 1.0 & 1.2 & 3.0 & 5.0 & 70 \\
\hline Copper, dissolved & 17 & 1 & 16 & $<1$ & e1.0 & $\mathrm{e} 1.0$ & e4.0 & 6.0 \\
\hline Iron, total recoverable & 28 & 10 & 28 & 130 & 285 & 405 & 697 & 43,000 \\
\hline Iron, dissolved & 26 & 3 & 26 & 10 & 20 & 35 & 57 & 480 \\
\hline Lead, total recoverable & 28 & 1 & 13 & $<1$ & $\mathrm{e} 0.1$ & $\mathrm{e} 0.6$ & $\mathrm{e} 2.8$ & 100 \\
\hline Lead, dissolved & 17 & 1 & 3 & $<1$ & -- & -- & -- & 4.0 \\
\hline Nickel, total recoverable & 28 & 1 & 25 & $<1$ & e2.0 & e3.0 & e12 & 150 \\
\hline Nickel, dissolved & 26 & 1 & 11 & $<1$ & $\mathrm{e} 0.3$ & $\mathrm{e} 0.5$ & $\mathrm{e} 1.0$ & 5.0 \\
\hline Zinc, total recoverable & 28 & 10 & 13 & $<10$ & e1.6 & e5.7 & e20 & 190 \\
\hline Zinc, dissolved & 17 & 3 & 11 & $<3$ & e2.4 & $\mathrm{e} 4.0$ & e6.5 & 11 \\
\hline \multicolumn{9}{|c|}{ After construction, January 1996 to April 1998 , trace metals $(\mu \mathrm{g} / \mathrm{L})$} \\
\hline Aluminum, total recoverable & 4 & 10 & 4 & 60 & -- & -- & -- & 200 \\
\hline Aluminum, dissolved & 4 & 10 & 1 & -- & -- & -- & -- & 9.3 \\
\hline Cadmium, total recoverable & 4 & 1 & 0 & -- & -- & -- & -- & -- \\
\hline Cadmium, dissolved & 0 & 1 & -- & -- & -- & -- & -- & -- \\
\hline Chromium, total recoverable & 4 & 1 & 1 & -- & -- & -- & -- & 2 \\
\hline Chromium, dissolved & 0 & 1 & -- & -- & -- & -- & -- & -- \\
\hline Copper, total recoverable & 4 & 1 & 1 & -- & -- & -- & -- & 1 \\
\hline Copper, dissolved & 0 & 1 & -- & -- & -- & -- & -- & -- \\
\hline Iron, total recoverable & 4 & 10 & 4 & 230 & -- & 430 & -- & 630 \\
\hline Iron, dissolved & 4 & 3 & 4 & 44 & -- & 54 & -- & 65 \\
\hline Lead, total recoverable & 4 & 1 & 0 & -- & -- & -- & -- & -- \\
\hline Lead, dissolved & 0 & 1 & -- & -- & -- & -- & -- & -- \\
\hline Nickel, total recoverable & 4 & 1 & 2 & -- & -- & -- & -- & -- \\
\hline Nickel, dissolved & 4 & 1 & 0 & -- & -- & -- & -- & -- \\
\hline Zinc, total recoverable & 4 & 10 & 0 & -- & -- & -- & -- & -- \\
\hline Zinc, dissolved & 0 & 20 & 0 & -- & -- & -- & -- & -- \\
\hline
\end{tabular}


Table 21. Statistical summary of selected water-quality data collected at station 273950, South Fork Kapunahala Stream at Kaneohe, Oahu, Hawaii, from May 1988 to April 1998

[inst., instantaneous; $\mu \mathrm{S} / \mathrm{cm}$ at $25^{\circ} \mathrm{C}$, microsiemens per centimeter at 25 degrees Celsius; $\mathrm{mg} / \mathrm{L}$, milligram per liter; $\mu \mathrm{g} / \mathrm{L}$, microgram per liter; <, less than; e, estimated using log-probability regression; $0.7 \mu \mathrm{m}-\mathrm{MF}, 0.7$ micron membrane filter; cols./100 mL, colonies per 100 milliliters; $\mathrm{ft}^{3} / \mathrm{s}$, cubic foot per second; ${ }^{\circ} \mathrm{C}$, degrees Celsius; NTU, nephelometric turbidity unit; --, no data or not computed; Range of dates are the time periods of water-quality data collection during the specified construction activity]

\begin{tabular}{|c|c|c|c|c|c|c|c|c|}
\hline Property or constituent & $\begin{array}{l}\text { Number } \\
\text { of } \\
\text { samples }\end{array}$ & $\begin{array}{l}\text { Minimum } \\
\text { detection } \\
\text { limit }\end{array}$ & $\begin{array}{l}\text { Number } \\
\text { of } \\
\text { detections }\end{array}$ & $\begin{array}{l}\text { Mini- } \\
\text { mum }\end{array}$ & \multicolumn{3}{|c|}{ Concentration percentile } & $\begin{array}{l}\text { Maxi- } \\
\text { mum }\end{array}$ \\
\hline \multicolumn{9}{|c|}{ Before construction, May 1988 to December 1988, physical, biological, and organic } \\
\hline Discharge, inst., $\left(\mathrm{ft}^{3} / \mathrm{s}\right)$ & 5 & 0 & 5 & 2.1 & -- & 2.8 & -- & 4.9 \\
\hline Specific conductance & & & & & & & & \\
\hline$\left(\mu \mathrm{S} / \mathrm{cm}\right.$ at $\left.25^{\circ} \mathrm{C}\right)$ & 5 & 1 & 5 & 190 & -- & 195 & -- & 200 \\
\hline $\mathrm{pH}$ (units) & 5 & 0.1 & 5 & 7.1 & -- & 7.7 & -- & 7.9 \\
\hline Temperature, water $\left({ }^{\circ} \mathrm{C}\right)$ & 5 & 0.5 & 5 & 22.5 & -- & 23.0 & -- & 23.3 \\
\hline Turbidity (NTU) & 5 & 0.1 & 5 & 2.4 & -- & 3.8 & -- & 41 \\
\hline Oxygen dissolved (mg/L) & 5 & 0.1 & 5 & 7.6 & -- & 7.9 & -- & 8.1 \\
\hline $\begin{array}{l}\text { Fecal coliform } 0.7 \mu \mathrm{m}-\mathrm{MF} \\
(\text { cols. } / 100 \mathrm{~mL})\end{array}$ & 3 & 0 & 3 & 2,200 & -- & -- & -- & 10,000 \\
\hline $\begin{array}{l}\text { Solids, residue at } 105^{\circ} \mathrm{C}, \\
\text { suspended }(\mathrm{mg} / \mathrm{L})\end{array}$ & 3 & 1 & 3 & 3 & -- & 8 & -- & 12 \\
\hline Carbon, organic, total $(\mathrm{mg} / \mathrm{L})$ & 2 & 0.1 & 2 & 1.7 & -- & -- & -- & 1.9 \\
\hline Oil and grease, total $(\mathrm{mg} / \mathrm{L})$ & 2 & 1 & 0 & -- & -- & -- & -- & -- \\
\hline \multicolumn{9}{|c|}{ During construction, March 1989 to December 1995, physical, biological, and organic } \\
\hline Discharge, inst., $\left(\mathrm{ft}^{3} / \mathrm{s}\right)$ & 72 & 0 & 72 & 1.6 & 1.9 & 2.1 & 2.4 & 99 \\
\hline $\begin{array}{l}\text { Specific conductance } \\
\left(\mu \mathrm{S} / \mathrm{cm} \text { at } 25^{\circ} \mathrm{C}\right)\end{array}$ & 71 & 1 & 71 & 160 & 190 & & 205 & \\
\hline $\mathrm{pH}$ (units) & 71 & 0.1 & 71 & 6.8 & 7.6 & $\begin{array}{r}198 \\
7.8\end{array}$ & 7.9 & 8.1 \\
\hline Temperature, water $\left({ }^{\circ} \mathrm{C}\right)$ & 71 & 0.5 & 71 & 20.0 & 21.5 & 22.5 & 23.0 & 25.5 \\
\hline Turbidity (NTU) & 71 & 0.1 & 71 & 0.3 & 2.3 & 3.3 & 4.3 & 71 \\
\hline Oxygen dissolved (mg/L) & 71 & 0.1 & 71 & 7.7 & 8.2 & 8.3 & 8.5 & 8.9 \\
\hline $\begin{array}{l}\text { Fecal coliform } 0.7 \mu \mathrm{m}-\mathrm{MF} \\
(\text { cols. } / 100 \mathrm{~mL})\end{array}$ & 28 & 0 & 28 & 500 & 1,125 & 1,850 & 4,975 & 46,000 \\
\hline $\begin{array}{l}\text { Solids, residue at } 105^{\circ} \mathrm{C}, \\
\text { suspended }(\mathrm{mg} / \mathrm{L})\end{array}$ & 29 & 1 & 26 & $<1$ & e3.0 & e9.0 & $\mathrm{e} 13.5$ & 144 \\
\hline Carbon, organic, total (mg/L) & ) 15 & 0.1 & 15 & 1.0 & 1.1 & 1.4 & 2.5 & 14 \\
\hline Oil and grease, total (mg/L) & 15 & 1 & 2 & 1 & -- & -- & -- & 2 \\
\hline \multicolumn{9}{|c|}{ After construction, January 1996 to April 1998, physical, biological, and organic } \\
\hline Discharge, inst., $\left(\mathrm{ft}^{3} / \mathrm{s}\right)$ & 18 & 0 & 18 & 1.6 & 1.8 & 2.0 & 2.3 & 3.6 \\
\hline Specific conductance & & & & & & & & \\
\hline$\left(\mu \mathrm{S} / \mathrm{cm}\right.$ at $\left.25^{\circ} \mathrm{C}\right)$ & 21 & 1 & 21 & 170 & 194 & 200 & 208 & 301 \\
\hline $\mathrm{pH}$ (units) & 21 & 0.1 & 21 & 6.8 & 7.7 & 7.9 & 8.0 & 8.1 \\
\hline Temperature, water $\left({ }^{\circ} \mathrm{C}\right)$ & 18 & 0.5 & 18 & 20.0 & 21.5 & 22.2 & 23.1 & 25.0 \\
\hline Turbidity (NTU) & 20 & 0.1 & 20 & 1.2 & 2.4 & 2.8 & 3.8 & 4.9 \\
\hline Oxygen dissolved (mg/L) & 20 & 0.1 & 20 & 8.1 & 8.2 & 8.4 & 8.7 & 8.8 \\
\hline $\begin{array}{l}\text { Fecal coliform } 0.7 \mu \mathrm{m}-\mathrm{MF} \\
(\text { cols. } / 100 \mathrm{~mL})\end{array}$ & 8 & 0 & 8 & 780 & 1,300 & 1,950 & 3,875 & 8,000 \\
\hline $\begin{array}{l}\text { Solids, residue at } 105^{\circ} \mathrm{C}, \\
\text { suspended }(\mathrm{mg} / \mathrm{L})\end{array}$ & 9 & 1 & 8 & $<1$ & e3.0 & $\mathrm{e} 4.0$ & e7.5 & 15 \\
\hline Carbon, organic, total $(\mathrm{mg} / \mathrm{L})$ & 4 & 0.1 & 4 & 1.3 & -- & -- & -- & 2.7 \\
\hline Oil and grease, total (mg/L) & 4 & 1 & 0 & -- & -- & -- & -- & -- \\
\hline
\end{tabular}


Table 21. Statistical summary of selected water-quality data collected at station 16273950 , South Fork Kapunahala Stream at Kaneohe, Oahu, Hawaii, from May 1988 to April 1998--Continued

\begin{tabular}{|c|c|c|c|c|c|c|c|c|}
\hline Property or constituent & $\begin{array}{c}\text { Number } \\
\text { of } \\
\text { samples }\end{array}$ & $\begin{array}{l}\text { Minimum } \\
\text { detection } \\
\text { limit }\end{array}$ & $\begin{array}{l}\text { Number } \\
\text { of } \\
\text { detections }\end{array}$ & $\begin{array}{l}\text { Mini- } \\
\text { mum }\end{array}$ & \multicolumn{3}{|c|}{ Concentration percentile } & $\begin{array}{l}\text { Maxi- } \\
\text { mum }\end{array}$ \\
\hline \multicolumn{9}{|c|}{ Before construction, May 1988 to December 1988, major ions and nutrients (mg/L) } \\
\hline Hardness, total (as $\left.\mathrm{CaCO}_{3}\right)$ & ) 2 & 1 & 2 & 57 & -- & -- & -- & 57 \\
\hline Calcium, dissolved & 2 & 0.02 & 2 & 12 & -- & -- & -- & 12 \\
\hline Magnesium, dissolved & 2 & 0.01 & 2 & 6.6 & -- & -- & -- & 6.6 \\
\hline Sodium, dissolved & 2 & 0.2 & 2 & 16 & -- & -- & -- & 17 \\
\hline Potassium, dissolved & 2 & 0.1 & 2 & 1.3 & -- & -- & -- & 1.6 \\
\hline Alkalinity $\left(\right.$ as $\mathrm{CaCO}_{3}$ ) & 2 & 1 & 2 & 57 & -- & -- & -- & 60 \\
\hline Sulfate, dissolved & 2 & 0.1 & 2 & 4.8 & -- & -- & -- & 7.8 \\
\hline Chloride, dissolved & 2 & 0.1 & 2 & 20 & -- & -- & -- & 20 \\
\hline Fluoride, dissolved & 2 & 0.1 & 2 & 0.1 & -- & -- & -- & 0.2 \\
\hline Silica, dissolved & 2 & 0.01 & 2 & 28 & -- & -- & -- & 30 \\
\hline $\begin{array}{l}\text { Nitrogen, nitrite plus } \\
\text { nitrate, total (as N) }\end{array}$ & 4 & 0.05 & 1 & -- & -- & -- & -- & 0.20 \\
\hline $\begin{array}{l}\text { Nitrogen, ammonia } \\
\text { plus organic, total (as N) }\end{array}$ & 4 & 0.2 & 3 & $<0.2$ & -- & -- & -- & 0.20 \\
\hline Phosphorus, total (as P) & 4 & 0.01 & 4 & 0.02 & -- & -- & -- & 0.04 \\
\hline \multicolumn{9}{|c|}{ During construction, March 1989 to December 1995, major ions and nutrients (mg/L) } \\
\hline Hardness, total $\left(\right.$ as $\left.\mathrm{CaCO}_{3}\right)$ & ) 15 & 1 & 15 & 40 & 55 & 57 & 60 & 69 \\
\hline Calcium, dissolved & 15 & 0.02 & 15 & 9.6 & 11 & 12 & 13 & 15 \\
\hline Magnesium, dissolved & 15 & 0.01 & 15 & 3.9 & 6.5 & 6.6 & 6.7 & 7.7 \\
\hline Sodium, dissolved & 15 & 0.2 & 15 & 12 & 16 & 17 & 17 & 18 \\
\hline Potassium, dissolved & 15 & 0.1 & 15 & 1.0 & 1.1 & 1.2 & 1.5 & 2.2 \\
\hline Alkalinity $\left(\right.$ as $\left.\mathrm{CaCO}_{3}\right)$ & 15 & 1 & 15 & 35 & 60 & 63 & 65 & 67 \\
\hline Sulfate, dissolved & 15 & 0.1 & 15 & 3.2 & 4.0 & 5.0 & 6.0 & 13 \\
\hline Chloride, dissolved & 15 & 0.1 & 15 & 18 & 19 & 20 & 21 & 26 \\
\hline Fluoride, dissolved & 15 & 0.1 & 5 & $<0.10$ & $\mathrm{e} 0.04$ & $\mathrm{e} 0.06$ & $\mathrm{e} 0.10$ & 0.20 \\
\hline Silica, dissolved & 15 & 0.01 & 15 & 11 & 29 & 30 & 32 & 33 \\
\hline $\begin{array}{l}\text { Nitrogen, nitrite plus } \\
\text { nitrate, total (as N) }\end{array}$ & 17 & 0.05 & 9 & $<0.05$ & $\mathrm{e} 0.02$ & $\mathrm{e} 0.08$ & $\mathrm{e} 0.37$ & 4.3 \\
\hline $\begin{array}{l}\text { Nitrogen, nitrite plus } \\
\text { nitrate, dissolved (as N) }\end{array}$ & 7 & 0.05 & 6 & $<0.05$ & $\mathrm{e} 0.05$ & $\mathrm{e} 0.09$ & $\mathrm{e} 0.11$ & 0.51 \\
\hline $\begin{array}{l}\text { Nitrogen, ammonia } \\
\text { plus organic, total (as N) }\end{array}$ & 29 & 0.2 & 7 & $<0.20$ & $\mathrm{e} 0.04$ & $\mathrm{e} 0.07$ & $\mathrm{e} 0.17$ & 0.70 \\
\hline Phosphorus, total (as P) & 29 & 0.01 & 26 & $<0.01$ & $\mathrm{e} 0.02$ & $\mathrm{e} 0.03$ & $\mathrm{e} 0.04$ & 0.29 \\
\hline \multicolumn{9}{|c|}{ After construction, January 1996 to April 1998, major ions and nutrients (mg/L) } \\
\hline Hardness, total (as $\left.\mathrm{CaCO}_{3}\right)$ & ) 4 & 1 & 4 & 56 & -- & 58 & -- & 61 \\
\hline Calcium, dissolved & 4 & 0.02 & 4 & 12 & -- & 12 & -- & 13 \\
\hline Magnesium, dissolved & 4 & 0.01 & 4 & 6.4 & -- & 6.6 & -- & 6.8 \\
\hline Sodium, dissolved & 4 & 0.2 & 4 & 15 & -- & 16 & -- & 17 \\
\hline Potassium, dissolved & 4 & 0.1 & 4 & 1.1 & -- & 1.2 & -- & 1.4 \\
\hline Alkalinity $\left(\right.$ as $\left.\mathrm{CaCO}_{3}\right)$ & 4 & 1 & 4 & 62 & -- & 63 & -- & 64 \\
\hline Sulfate, dissolved & 4 & 0.1 & 4 & 4.9 & -- & 6.6 & -- & 8.3 \\
\hline Chloride, dissolved & 4 & 0.1 & 4 & 18 & -- & 19 & -- & 20 \\
\hline Fluoride, dissolved & 4 & 0.1 & 0 & -- & -- & -- & -- & -- \\
\hline Silica, dissolved & 4 & 0.01 & 4 & 30 & -- & 31 & -- & 32 \\
\hline $\begin{array}{l}\text { Nitrogen, nitrite plus } \\
\text { nitrate, dissolved (as N) }\end{array}$ & 9 & 0.05 & 8 & $<0.05$ & $\mathrm{e} 0.06$ & $\mathrm{e} 0.08$ & $\mathrm{e} 0.10$ & 0.13 \\
\hline $\begin{array}{l}\text { Nitrogen, ammonia } \\
\text { plus organic, total (as } \mathrm{N} \text { ) }\end{array}$ & 9 & 0.1 & 0 & -- & -- & -- & -- & -- \\
\hline Phosphorus, total (as P) & 9 & 0.05 & 9 & 0.02 & 0.02 & 0.02 & 0.03 & 0.04 \\
\hline
\end{tabular}


Table 21. Statistical summary of selected water-quality data collected at station 16273950, South Fork Kapunahala Stream at Kaneohe, Oahu, Hawaii, from May 1988 to April 1998--Continued

\begin{tabular}{|c|c|c|c|c|c|c|c|c|}
\hline \multirow[b]{2}{*}{ Property or constituent } & \multirow{2}{*}{$\begin{array}{l}\text { Number } \\
\text { of } \\
\text { samples }\end{array}$} & \multirow{2}{*}{$\begin{array}{l}\text { Minimum } \\
\text { detection } \\
\text { limit }\end{array}$} & \multirow{2}{*}{$\begin{array}{l}\text { Number } \\
\text { of } \\
\text { detections }\end{array}$} & \multirow{2}{*}{$\begin{array}{l}\text { Mini- } \\
\text { mum }\end{array}$} & \multicolumn{3}{|c|}{ Concentration percentile } & \multirow{2}{*}{$\begin{array}{l}\text { Maxi- } \\
\text { mum }\end{array}$} \\
\hline & & & & & 25 & 50 & 75 & \\
\hline \multicolumn{9}{|c|}{ Before construction, May 1988 to December 1988, trace metals $(\mu \mathrm{g} / \mathrm{L})$} \\
\hline Aluminum, total recoverable & e 2 & 10 & 2 & 200 & -- & -- & -- & 320 \\
\hline Aluminum, dissolved & 2 & 10 & 2 & 10 & -- & -- & -- & 20 \\
\hline Cadmium, total recoverable & 2 & 1 & 0 & -- & -- & -- & -- & -- \\
\hline Cadmium, dissolved & 2 & 1 & 1 & -- & -- & -- & -- & 1.0 \\
\hline Chromium, total recoverable & 2 & 10 & 2 & 1.0 & -- & -- & -- & 4.0 \\
\hline Chromium, dissolved & 2 & 1 & 0 & -- & -- & -- & -- & -- \\
\hline Copper, total recoverable & 2 & 1 & 2 & 4.0 & -- & -- & -- & 6.0 \\
\hline Copper, dissolved & 2 & 1 & 2 & 2.0 & -- & -- & -- & 2.0 \\
\hline Iron, total recoverable & 2 & 10 & 2 & 740 & -- & -- & -- & 1,900 \\
\hline Iron, dissolved & 2 & 3 & 2 & 210 & -- & -- & -- & 320 \\
\hline Lead, total recoverable & 2 & 1 & 1 & -- & -- & -- & -- & 5.0 \\
\hline Lead, dissolved & 2 & 1 & 0 & -- & -- & -- & -- & -- \\
\hline Nickel, total recoverable & 2 & 1 & 2 & 1 & -- & -- & -- & 5.0 \\
\hline Nickel, dissolved & 2 & 1 & 1 & -- & -- & -- & -- & 3.0 \\
\hline Zinc, total recoverable & 2 & 10 & 0 & -- & -- & -- & -- & -- \\
\hline Zinc, dissolved & 2 & 3 & 0 & -- & -- & -- & -- & -- \\
\hline \multicolumn{9}{|c|}{ During construction, March 1989 to December 1995, trace metals $(\mu \mathrm{g} / \mathrm{L})$} \\
\hline Aluminum, total recoverable & e 15 & 10 & 15 & 60 & 180 & 270 & 300 & 7,200 \\
\hline Aluminum, dissolved & 15 & 10 & 10 & $<10$ & e6.9 & e10 & e30 & 40 \\
\hline Cadmium, total recoverable & 15 & 1 & 1 & -- & -- & -- & -- & 3 \\
\hline Cadmium, dissolved & 6 & 1 & 0 & -- & -- & -- & -- & -- \\
\hline Chromium, total recoverable & e 15 & 1 & 14 & $<1$ & $\mathrm{e} 2.0$ & $\mathrm{e} 2.0$ & e3.0 & 40 \\
\hline Chromium, dissolved & 6 & 1 & 0 & -- & -- & -- & -- & -- \\
\hline Copper, total recoverable & 15 & 1 & 12 & $<1$ & $\mathrm{e} 1.0$ & $\mathrm{e} 2.0$ & $\mathrm{e} 2.0$ & 13.0 \\
\hline Copper, dissolved & 6 & 1 & 4 & 1.0 & -- & -- & -- & 3.0 \\
\hline Iron, total recoverable & 15 & 10 & 15 & 370 & 680 & 790 & 860 & 14,000 \\
\hline Iron, dissolved & 15 & 3 & 15 & 42 & 81 & 110 & 140 & 200 \\
\hline Lead, total recoverable & 15 & 1 & 4 & $<1$ & -- & -- & -- & 12 \\
\hline Lead, dissolved & 6 & 1 & 2 & $<1$ & -- & -- & -- & 2.0 \\
\hline Nickel, total recoverable & 15 & 1 & 10 & $<10$ & $\mathrm{e} 0.28$ & $\mathrm{e} 1.0$ & $\mathrm{e} 2.0$ & 29 \\
\hline Nickel, dissolved & 15 & 1 & 1 & $<1$ & -- & -- & -- & 2.0 \\
\hline Zinc, total recoverable & 15 & 10 & 4 & $<10$ & -- & -- & -- & 40 \\
\hline Zinc, dissolved & 6 & 3 & 4 & $<3$ & -- & -- & -- & 100 \\
\hline \multicolumn{9}{|c|}{ After construction, January 1996 to April 1998 , trace metals $(\mu \mathrm{g} / \mathrm{L})$} \\
\hline Aluminum, total recoverable & e 4 & 10 & 4 & 70 & -- & -- & -- & 210 \\
\hline Aluminum, dissolved & 4 & 10 & 2 & $<2$ & -- & -- & -- & 7.7 \\
\hline Cadmium, total recoverable & 4 & 1 & 0 & -- & -- & -- & -- & -- \\
\hline Cadmium, dissolved & 0 & 1 & -- & -- & -- & -- & -- & -- \\
\hline Chromium, total recoverable & 4 & 1 & 3 & $<1$ & -- & -- & -- & 3 \\
\hline Chromium, dissolved & 0 & 1 & -- & -- & -- & -- & -- & -- \\
\hline Copper, total recoverable & 4 & 1 & 3 & -- & -- & -- & -- & 2 \\
\hline Copper, dissolved & 0 & 1 & -- & -- & -- & -- & -- & -- \\
\hline Iron, total recoverable & 4 & 10 & 4 & 220 & -- & -- & -- & 760 \\
\hline Iron, dissolved & 4 & 3 & 4 & 40 & -- & -- & -- & 84 \\
\hline Lead, total recoverable & 4 & 1 & 0 & -- & -- & -- & -- & -- \\
\hline Lead, dissolved & 0 & 1 & -- & -- & -- & -- & -- & -- \\
\hline Nickel, total recoverable & 4 & 1 & 0 & -- & -- & -- & -- & -- \\
\hline Nickel, dissolved & 0 & 1 & -- & -- & -- & -- & -- & -- \\
\hline Zinc, total recoverable & 4 & 10 & 0 & -- & -- & -- & -- & -- \\
\hline Zinc, dissolved & 0 & 20 & -- & -- & -- & -- & -- & -- \\
\hline
\end{tabular}


Table 22. Statistical summary of selected water-quality data collected at station 16274100 , Kaneohe Stream below Kamehameha Highway at Kaneohe, Oahu, Hawaii, from November 1988 to August 1999

[inst., instantaneous; $\mu \mathrm{S} / \mathrm{cm}$ at $25^{\circ} \mathrm{C}$, microsiemens per centimeter at 25 degrees Celsius; $\mathrm{mg} / \mathrm{L}$, milligram per liter; $\mu \mathrm{g} / \mathrm{L}$, microgram per liter; <, less than; e, estimated using log-probability regression; $0.7 \mu \mathrm{m}-\mathrm{MF}, 0.7$ micron membrane filter; cols. $/ 100 \mathrm{~mL}$, colonies per 100 milliliters; $\mathrm{ft}^{3} / \mathrm{s}$, cubic foot per second; ${ }^{\circ} \mathrm{C}$, degrees Celsius; NTU, nephelometric turbidity unit; --, no data or not computed; Range of dates are the time periods of water-quality data collection during the specified construction activity]

\begin{tabular}{|c|c|c|c|c|c|c|c|c|}
\hline Property or constituent & $\begin{array}{l}\text { Number } \\
\text { of } \\
\text { samples }\end{array}$ & $\begin{array}{l}\text { Minimum } \\
\text { detection } \\
\text { limit }\end{array}$ & $\begin{array}{l}\text { Number } \\
\text { of } \\
\text { detections }\end{array}$ & $\begin{array}{l}\text { Mini- } \\
\text { mum }\end{array}$ & \multicolumn{3}{|c|}{ Concentration percentile } & $\begin{array}{l}\text { Maxi- } \\
\text { mum }\end{array}$ \\
\hline \multicolumn{9}{|c|}{ During construction, November 1988 to November 1995, physical, biological, and organic } \\
\hline Discharge, inst., $\left(\mathrm{ft}^{3} / \mathrm{s}\right)$ & 73 & 0 & 73 & 7.5 & 10.5 & 13.0 & 16.5 & 44 \\
\hline \multicolumn{9}{|l|}{ Specific conductance } \\
\hline$\left(\mu \mathrm{S} / \mathrm{cm}\right.$ at $\left.25^{\circ} \mathrm{C}\right)$ & 74 & 1 & 74 & 120 & 190 & 198 & 202 & 225 \\
\hline $\mathrm{pH}$ (units) & 73 & 0.1 & 73 & 6.7 & 8.3 & 8.6 & 8.8 & 9.3 \\
\hline Temperature, water $\left({ }^{\circ} \mathrm{C}\right)$ & 74 & 0.5 & 74 & 20.0 & 23.0 & 25.0 & 26.6 & 31.0 \\
\hline Turbidity (NTU) & 74 & 0.1 & 74 & 1.1 & 2.2 & 3.4 & 5.7 & 38 \\
\hline Oxygen dissolved (mg/L) & 74 & 0.1 & 74 & 5.9 & 8.8 & 9.2 & 9.6 & 10 \\
\hline \multicolumn{8}{|l|}{ Fecal coliform $0.7 \mu \mathrm{m}-\mathrm{MF}$} & 20,000 \\
\hline \multicolumn{9}{|l|}{ Solids, residue at $105^{\circ} \mathrm{C}$} \\
\hline Carbon, organic, total $(\mathrm{mg} / \mathrm{L})$ & 14 & 0.1 & 14 & 1.3 & 1.8 & 2.2 & 2.7 & 3.3 \\
\hline Oil and grease, total (mg/L) & 14 & 1 & 1 & -- & -- & -- & -- & 2 \\
\hline \multicolumn{9}{|c|}{ After construction, January 1996 to August 1999, physical, biological, and organic } \\
\hline Discharge, inst., $\left(\mathrm{ft}^{3} / \mathrm{s}\right)$ & 19 & 0 & 19 & 8.0 & 11 & 15 & 18 & 50 \\
\hline $\begin{array}{l}\text { Specific conductance } \\
\left(\mu \mathrm{S} / \mathrm{cm} \text { at } 25^{\circ} \mathrm{C}\right)\end{array}$ & 20 & 1 & 20 & 194 & 199 & 204 & 209 & 213 \\
\hline $\mathrm{pH}$ (units) & 19 & 0.1 & 19 & 7.9 & 8.4 & 8.6 & 9.0 & 9.3 \\
\hline Temperature, water $\left({ }^{\circ} \mathrm{C}\right)$ & 20 & 0.5 & 20 & 21.0 & 22.0 & 24.5 & 26.4 & 21.0 \\
\hline Turbidity (NTU) & 18 & 0.1 & 18 & 0.80 & 2.0 & 2.4 & 4.4 & 34 \\
\hline Oxygen dissolved (mg/L) & 20 & 0.1 & 20 & 8.8 & 9.2 & 9.6 & 9.9 & 10.8 \\
\hline $\begin{array}{l}\text { Fecal coliform } 0.7 \mu \mathrm{m}-\mathrm{MF} \\
(\text { cols } / 100 \mathrm{~mL})\end{array}$ & 8 & 0 & 8 & 880 & 1,250 & 2,800 & 4,925 & 5,200 \\
\hline $\begin{array}{l}\text { Solids, residue at } 105^{\circ} \mathrm{C}, \\
\text { suspended }(\mathrm{mg} / \mathrm{L})\end{array}$ & 9 & 1 & 8 & $<1$ & $\mathrm{e} 3.5$ & e6.0 & e6.0 & 20 \\
\hline Carbon, organic, total $(\mathrm{mg} / \mathrm{L})$ & 5 & 0.1 & 5 & 1.5 & -- & 3.2 & -- & 5.0 \\
\hline Oil and grease, total (mg/L) & 5 & 1 & 0 & -- & -- & -- & -- & -- \\
\hline
\end{tabular}


Table 22. Statistical summary of selected water-quality data collected at station 16274100 , Kaneohe Stream below Kamehameha Highway at Kaneohe, Oahu, Hawaii, from November 1988 to August 1999--Continued

\begin{tabular}{|c|c|c|c|c|c|c|c|c|}
\hline Property or constituent & $\begin{array}{l}\text { Number } \\
\text { of } \\
\text { samples }\end{array}$ & $\begin{array}{l}\text { Minimum } \\
\text { detection } \\
\text { limit }\end{array}$ & $\begin{array}{l}\text { Number } \\
\text { of } \\
\text { detections }\end{array}$ & $\begin{array}{l}\text { Mini- } \\
\text { mum }\end{array}$ & $\frac{\text { Conce }}{25}$ & $\frac{\text { ation pe }}{50}$ & $\frac{\text { entile }}{75}$ & $\begin{array}{l}\text { Maxi- } \\
\text { mum }\end{array}$ \\
\hline \multicolumn{9}{|c|}{ During construction, November 1988 to November 1995, major ions and nutrients (mg/L) } \\
\hline Hardness, total (as $\left.\mathrm{CaCO}_{3}\right)$ & 14 & 1 & 14 & 54 & 56 & 58 & 61 & 63 \\
\hline Calcium, dissolved & 14 & 0.02 & 14 & 10 & 10 & 11 & 12 & 10 \\
\hline Magnesium, dissolved & 14 & 0.01 & 14 & 6.6 & 7.1 & 7.4 & 7.6 & 8.1 \\
\hline Sodium, dissolved & 14 & 0.2 & 14 & 13 & 15 & 16 & 16 & 17 \\
\hline Potassium, dissolved & 14 & 0.1 & 14 & 0.90 & 1.0 & 1.1 & 1.3 & 1.7 \\
\hline Alkalinity $\left(\right.$ as $\mathrm{CaCO}_{3}$ ) & 14 & 1 & 14 & 50 & 54 & 57 & 60 & 63 \\
\hline Sulfate, dissolved & 14 & 0.1 & 14 & 3.4 & 6.6 & 7.2 & 9.2 & 12 \\
\hline Chloride, dissolved & 14 & 0.1 & 14 & 18 & 18 & 20 & 22 & 27 \\
\hline Fluoride, dissolved & 14 & 0.1 & 5 & $<0.1$ & $\mathrm{e} 0.04$ & $\mathrm{e} 0.06$ & $\mathrm{e} 0.10$ & 0.20 \\
\hline Silica, dissolved & 14 & 0.01 & 14 & 16 & 20 & 22 & 23 & 24 \\
\hline $\begin{array}{l}\text { Nitrogen, nitrite plus } \\
\text { nitrate, total (as N) }\end{array}$ & 18 & 0.05 & 18 & 0.17 & 0.27 & 0.30 & 0.49 & 0.79 \\
\hline $\begin{array}{l}\text { Nitrogen, nitrite plus } \\
\text { nitrate, dissolved (as N) }\end{array}$ & 7 & 0.05 & 7 & 0.15 & 0.19 & 0.34 & 0.35 & 0.44 \\
\hline $\begin{array}{l}\text { Nitrogen, ammonia } \\
\text { plus organic, total (as } \mathrm{N} \text { ) }\end{array}$ & 29 & 0.2 & 15 & $<0.2$ & $\mathrm{e} 0.13$ & $\mathrm{e} 0.20$ & $\mathrm{e} 0.30$ & 0.60 \\
\hline Phosphorus, total (as P) & 29 & 0.01 & 27 & $<0.01$ & $\mathrm{e} 0.02$ & $\mathrm{e} 0.03$ & $\mathrm{e} 0.04$ & 0.10 \\
\hline \multicolumn{9}{|c|}{ After construction, January 1996 to August 1999, major ions and nutrients (mg/L) } \\
\hline Hardness, total (as $\left.\mathrm{CaCO}_{3}\right)$ & ) 6 & 1 & 6 & 66 & 60 & 62 & 64 & 66 \\
\hline Calcium, dissolved & 6 & 0.02 & 6 & 10 & 11 & 12 & 12 & 14 \\
\hline Magnesium, dissolved & 6 & 0.01 & 6 & 7.5 & 7.5 & 8.0 & 8.2 & 8.2 \\
\hline Sodium, dissolved & 6 & 0.2 & 6 & 15 & 15 & 16 & 16 & 16 \\
\hline Potassium, dissolved & 6 & 0.1 & 6 & 0.90 & 0.98 & 1.2 & 1.4 & 1.7 \\
\hline Alkalinity $\left(\right.$ as $\mathrm{CaCO}_{3}$ ) & 5 & 1 & 5 & 63 & -- & 63 & -- & 65 \\
\hline Sulfate, dissolved & 6 & 0.1 & 6 & 5.5 & 6.2 & 8.4 & 9.6 & 9.6 \\
\hline Chloride, dissolved & 6 & 0.1 & 6 & 18 & 19 & 20 & 20 & 20 \\
\hline Fluoride, dissolved & 6 & 0.1 & 0 & -- & -- & -- & -- & -- \\
\hline Silica, dissolved & 6 & 0.01 & 6 & 21 & 21 & 21 & 22 & 23 \\
\hline $\begin{array}{l}\text { Nitrogen, nitrite plus } \\
\text { nitrate, dissolved (as N) } \\
\text { Nitrogen, ammonia }\end{array}$ & 10 & 0.05 & 10 & 0.10 & 0.18 & 0.27 & 0.33 & 0.40 \\
\hline plus organic, total (as N) & 10 & 0.1 & 7 & $<0.1$ & $\mathrm{e} 0.13$ & $\mathrm{e} 0.20$ & $\mathrm{e} 0.32$ & 0.47 \\
\hline Phosphorus, total (as P) & 10 & 0.05 & 7 & $<0.05$ & $\mathrm{e} 0.01$ & $\mathrm{e} 0.03$ & $\mathrm{e} 0.04$ & 0.04 \\
\hline
\end{tabular}


Table 22. Statistical summary of selected water-quality data collected at station 16274100 , Kaneohe Stream below Kamehameha Highway at Kaneohe, Oahu, Hawaii, from November 1988 to August 1999--Continued

\begin{tabular}{|c|c|c|c|c|c|c|c|c|}
\hline Property or constituent & $\begin{array}{l}\text { Number } \\
\text { of } \\
\text { samples }\end{array}$ & $\begin{array}{l}\text { Minimum } \\
\text { detection } \\
\text { limit }\end{array}$ & $\begin{array}{l}\text { Number } \\
\text { of } \\
\text { detections }\end{array}$ & $\begin{array}{l}\text { Mini- } \\
\text { mum }\end{array}$ & $\frac{\text { Conc }}{25}$ & $\frac{\text { tration }}{50}$ & $\frac{\text { rcentile }}{75}$ & $\begin{array}{l}\text { Maxi- } \\
\text { mum }\end{array}$ \\
\hline \multicolumn{9}{|c|}{ During construction, November 1988 to November 1995, trace metals $(\mu \mathrm{g} / \mathrm{L})$} \\
\hline Aluminum, total recoverable & le 14 & 10 & 14 & 90 & 262 & 370 & 482 & 1,300 \\
\hline Aluminum, dissolved & 14 & 10 & 13 & $<10$ & e18 & e20 & e32 & 70 \\
\hline Cadmium, total recoverable & 14 & 1 & 0 & -- & -- & -- & -- & -- \\
\hline Cadmium, dissolved & 6 & 1 & 0 & -- & -- & -- & -- & -- \\
\hline Chromium, total recoverable & le 14 & 1 & 10 & $<1$ & $\mathrm{e} 0.84$ & $\mathrm{e} 2.0$ & e3.0 & 7.0 \\
\hline Chromium, dissolved & 6 & 1 & 1 & -- & -- & -- & -- & 2.0 \\
\hline Copper, total recoverable & 14 & 1 & 14 & 1.0 & 2.0 & 3.0 & 3.2 & 8.0 \\
\hline Copper, dissolved & 6 & 1 & 6 & 1.0 & 1.0 & 1.0 & 3.2 & 4.0 \\
\hline Iron, total recoverable & 14 & 10 & 14 & 350 & 565 & 840 & 1,100 & 3,100 \\
\hline Iron, dissolved & 14 & 3 & 14 & 13 & 54 & 97 & 110 & 140 \\
\hline Lead, total recoverable & 14 & 1 & 11 & $<1$ & $\mathrm{e} 0.89$ & $\mathrm{e} 1.0$ & $\mathrm{e} 2.0$ & 4.0 \\
\hline Lead, dissolved & 6 & 1 & 1 & -- & -- & -- & -- & 1.0 \\
\hline Nickel, total recoverable & 14 & 1 & 13 & $<1$ & $\mathrm{e} 1.0$ & $\mathrm{e} 2.0$ & $\mathrm{e} 2.2$ & 4.0 \\
\hline Nickel, dissolved & 14 & 1 & 3 & $<1$ & -- & -- & -- & 2.0 \\
\hline Zinc, total recoverable & 14 & 10 & 4 & $<10$ & -- & -- & -- & 20 \\
\hline Zinc, dissolved & 6 & 20 & 3 & $<3$ & -- & -- & -- & 7.0 \\
\hline \multicolumn{9}{|c|}{ After construction, January 1996 to August 1999 , trace metals $(\mu \mathrm{g} / \mathrm{L})$} \\
\hline Aluminum, total recoverable & le 5 & 10 & 5 & 70 & -- & 170 & -- & 700 \\
\hline Aluminum, dissolved & 6 & 10 & 5 & 7 & -- & 68 & -- & 130 \\
\hline Cadmium, total recoverable & 5 & 1 & 0 & -- & -- & -- & -- & -- \\
\hline Cadmium, dissolved & 1 & 1 & 0 & -- & -- & -- & -- & -- \\
\hline Chromium, total recoverable & 5 & 1 & 3 & $<1$ & -- & -- & -- & 2 \\
\hline Chromium, dissolved & 1 & 1 & 0 & -- & -- & -- & -- & -- \\
\hline Copper, total recoverable & 5 & 1 & 5 & 1 & -- & 2 & -- & 3 \\
\hline Copper, dissolved & 1 & 1 & 1 & -- & -- & -- & -- & 1 \\
\hline Iron, total recoverable & 5 & 10 & 5 & 310 & -- & 520 & -- & 1,200 \\
\hline Iron, dissolved & 6 & 3 & 6 & 60 & e68 & e95 & -- & e145 \\
\hline Lead, total recoverable & 5 & 1 & 1 & -- & -- & -- & -- & 1 \\
\hline Lead, dissolved & 1 & 1 & 0 & -- & -- & -- & -- & -- \\
\hline Nickel, total recoverable & 5 & 1 & 3 & $<1$ & -- & -- & -- & 2 \\
\hline Nickel, dissolved & 6 & 1 & 0 & -- & -- & -- & -- & -- \\
\hline Zinc, total recoverable & 5 & 10 & 1 & -- & -- & -- & -- & 13 \\
\hline Zinc, dissolved & 1 & 20 & 0 & -- & -- & -- & -- & -- \\
\hline
\end{tabular}


Table 23. Statistical summary of selected water-quality data collected at station 16275000, Haiku Stream near Heeia, Oahu, Hawaii, from March 1983 to April 1998

[inst., instantaneous; $\mu \mathrm{S} / \mathrm{cm}$ at $25^{\circ} \mathrm{C}$, microsiemens per centimeter at 25 degrees Celsius; $\mathrm{mg} / \mathrm{L}$, milligram per liter; $\mu \mathrm{g} / \mathrm{L}$, microgram per liter; <, less than; e, estimated using log-probability regression; $0.7 \mu \mathrm{m}-\mathrm{MF}, 0.7$ micron membrane filter; cols./100 mL, colonies per 100 milliliters; $\mathrm{ft}^{3} / \mathrm{s}$, cubic foot per second; ${ }^{\circ} \mathrm{C}$, degrees Celsius; NTU, nephelometric turbidity unit; --, no data or not computed; Range of dates are the time periods of water-quality data collection during the specified construction activity]

\begin{tabular}{|c|c|c|c|c|c|c|c|c|}
\hline Property or constituent & $\begin{array}{c}\text { Number } \\
\text { of } \\
\text { samples }\end{array}$ & $\begin{array}{l}\text { Minimum } \\
\text { detection } \\
\text { limit }\end{array}$ & $\begin{array}{l}\text { Number } \\
\text { of } \\
\text { detections }\end{array}$ & $\begin{array}{l}\text { Mini- } \\
\text { mum }\end{array}$ & $\frac{\text { Conc }}{25}$ & $\frac{\text { tration } \mathrm{p}}{50}$ & $\frac{\text { centile }}{75}$ & $\begin{array}{l}\text { Maxi- } \\
\text { mum }\end{array}$ \\
\hline \multicolumn{9}{|c|}{ Before construction, March 1983 to September 1988, physical, biological, and organic } \\
\hline Discharge, inst., $\left(\mathrm{ft}^{3} / \mathrm{s}\right)$ & 35 & 0 & 35 & 1.0 & 1.3 & 1.5 & 2.0 & 5.5 \\
\hline \multicolumn{9}{|l|}{ Specific conductance } \\
\hline$\left(\mu \mathrm{S} / \mathrm{cm}\right.$ at $\left.25^{\circ} \mathrm{C}\right)$ & 28 & 1 & 28 & 140 & 145 & 148 & 159 & 185 \\
\hline $\mathrm{pH}$ (units) & 28 & 0.1 & 28 & 6.3 & 7.0 & 7.3 & 7.7 & 7.9 \\
\hline Temperature, water $\left({ }^{\circ} \mathrm{C}\right)$ & 35 & 0.5 & 35 & 19.5 & 20.5 & 21.0 & 21.5 & 23.0 \\
\hline Turbidity (NTU) & 15 & 0.1 & 15 & 0.2 & 0.5 & 0.7 & 0.9 & 1.6 \\
\hline Oxygen dissolved (mg/L) & 15 & 0.1 & 15 & 5.5 & 8.0 & 8.3 & 8.6 & 8.6 \\
\hline $\begin{array}{l}\text { Fecal coliform } 0.7 \mu \mathrm{m}-\mathrm{MF} \\
(\text { cols. } / 100 \mathrm{~mL})\end{array}$ & 9 & 0 & 9 & 23 & 66 & 92 & 103 & 130 \\
\hline $\begin{array}{l}\text { Solids, residue at } 105^{\circ} \mathrm{C}, \\
\text { suspended }(\mathrm{mg} / \mathrm{L})\end{array}$ & 13 & 1 & 8 & $<1$ & e1.7 & e3.0 & e9.0 & 11.0 \\
\hline Carbon, organic, total $(\mathrm{mg} / \mathrm{L})$ & L) 6 & 0.1 & 6 & 0.2 & 0.4 & 0.5 & 0.6 & 0.8 \\
\hline Oil and grease, total $(\mathrm{mg} / \mathrm{L})$ & 6 & 1 & 1 & -- & -- & -- & -- & 2 \\
\hline \multicolumn{9}{|c|}{ During construction, November 1988 to November 1994, physical, biological, and organic } \\
\hline Discharge, inst., $\left(\mathrm{ft}^{3} / \mathrm{s}\right)$ & 61 & 0 & 61 & 1.1 & 1.5 & 1.7 & 2.0 & 33 \\
\hline $\begin{array}{l}\text { Specific conductance } \\
\left(\mu \mathrm{S} / \mathrm{cm} \text { at } 25^{\circ} \mathrm{C}\right)\end{array}$ & 61 & 1 & 61 & 92 & 145 & 150 & 154 & 185 \\
\hline $\mathrm{pH}$ (units) & 61 & 0.1 & 61 & 6.5 & 7.6 & 7.8 & 7.9 & 8.2 \\
\hline Temperature, water $\left({ }^{\circ} \mathrm{C}\right)$ & 63 & 0.5 & 63 & 18.5 & 20.0 & 20.5 & 21.0 & 22.5 \\
\hline Turbidity (NTU) & 61 & 0.1 & 61 & 0.1 & 0.4 & 0.7 & 1.2 & 500 \\
\hline Oxygen dissolved (mg/L) & 61 & 0.1 & 61 & 8.2 & 8.6 & 8.7 & 8.8 & 9.0 \\
\hline $\begin{array}{l}\text { Fecal coliform } 0.7 \mu \mathrm{m}-\mathrm{MF} \\
(\text { cols. } / 100 \mathrm{~mL})\end{array}$ & 24 & 0 & 24 & 34 & 132 & 220 & 382 & 8,100 \\
\hline $\begin{array}{l}\text { Solids, residue at } 105^{\circ} \mathrm{C}, \\
\text { suspended }(\mathrm{mg} / \mathrm{L})\end{array}$ & 28 & 1 & 18 & $<1$ & $\mathrm{e} 0.6$ & $\mathrm{e} 5.0$ & e9.0 & 201 \\
\hline Carbon, organic, total $(\mathrm{mg} / \mathrm{L})$ & L) 13 & 0.1 & 13 & 0.2 & 0.4 & 0.5 & 1.4 & 6.8 \\
\hline Oil and grease, total $(\mathrm{mg} / \mathrm{L})$ & 13 & 1 & 0 & -- & -- & -- & -- & -- \\
\hline \multicolumn{9}{|c|}{ After construction, December 1994 to April 1998, physical, biological, and organic } \\
\hline Discharge, inst., $\left(\mathrm{ft}^{3} / \mathrm{s}\right)$ & 28 & 0 & 28 & 1.2 & 1.4 & 1.7 & 2.0 & 16 \\
\hline Specific conductance & & & & & & & & \\
\hline$\left(\mu \mathrm{S} / \mathrm{cm}\right.$ at $\left.25^{\circ} \mathrm{C}\right)$ & 32 & 1 & 32 & 114 & 146 & 150 & 154 & 165 \\
\hline $\mathrm{pH}$ (units) & 32 & 0.1 & 32 & 7.3 & 7.6 & 7.7 & 7.8 & 8.0 \\
\hline Temperature, water $\left({ }^{\circ} \mathrm{C}\right)$ & 29 & 0.5 & 29 & 18.0 & 20.0 & 20.0 & 20.5 & 22.5 \\
\hline Turbidity (NTU) & 31 & 0.1 & 31 & 0.2 & 0.3 & 0.3 & 0.5 & 67 \\
\hline Oxygen dissolved (mg/L) & 32 & 0.1 & 32 & 8.4 & 8.7 & 8.8 & 9.1 & 9.3 \\
\hline $\begin{array}{l}\text { Fecal coliform } 0.7 \mu \mathrm{m}-\mathrm{MF} \\
(\text { cols. } / 100 \mathrm{~mL})\end{array}$ & 11 & 0 & 11 & 36 & 68 & 140 & 210 & 3,400 \\
\hline $\begin{array}{l}\text { Solids, residue at } 105^{\circ} \mathrm{C}, \\
\text { suspended }(\mathrm{mg} / \mathrm{L})\end{array}$ & 12 & 1 & 6 & $<1$ & $\mathrm{e} 0.3$ & $\mathrm{e} 0.7$ & $\mathrm{e} 1.0$ & 4.0 \\
\hline Carbon, organic, total $(\mathrm{mg} / \mathrm{L})$ & L) 6 & 0.1 & 6 & 0.3 & 0.4 & 0.4 & 1.0 & 1.5 \\
\hline Oil and grease, total $(\mathrm{mg} / \mathrm{L})$ & 6 & 1 & 1 & -- & -- & -- & -- & 1 \\
\hline
\end{tabular}


Table 23. Statistical summary of selected water-quality data collected at station 16275000, Haiku Stream near Heeia, Oahu, Hawaii, from March 1983 to April 1998--Continued

\begin{tabular}{|c|c|c|c|c|c|c|c|c|}
\hline \multirow[b]{2}{*}{ Property or constituent } & \multirow{2}{*}{$\begin{array}{l}\text { Number } \\
\text { of } \\
\text { samples }\end{array}$} & \multirow{2}{*}{$\begin{array}{l}\text { Minimum } \\
\text { detection } \\
\text { limit }\end{array}$} & \multirow{2}{*}{$\begin{array}{l}\text { Number } \\
\text { of } \\
\text { detections }\end{array}$} & \multirow{2}{*}{$\begin{array}{l}\text { Mini- } \\
\text { mum }\end{array}$} & \multicolumn{3}{|c|}{ Concentration percentile } & \multirow{2}{*}{$\begin{array}{l}\text { Maxi- } \\
\text { mum }\end{array}$} \\
\hline & & & & & 25 & 50 & 75 & \\
\hline \multicolumn{9}{|c|}{ Before construction, March 1983 to September 1988, major ions and nutrients $(\mathrm{mg} / \mathrm{L})$} \\
\hline Hardness, total (as $\mathrm{CaCO}_{3}$ ) & 6 & 1 & 6 & 45 & 45 & 46 & 47 & 48 \\
\hline Calcium, dissolved & 6 & 0.02 & 6 & 8.3 & 8.4 & 8.6 & 9.2 & 9.5 \\
\hline Magnesium, dissolved & 6 & 0.01 & 6 & 5.5 & 5.6 & 5.8 & 6.1 & 6.2 \\
\hline Sodium, dissolved & 6 & 0.2 & 6 & 12 & 12 & 12 & 12 & 12 \\
\hline Potassium, dissolved & 6 & 0.1 & 6 & 0.7 & 0.7 & 0.8 & 1 & 1 \\
\hline Alkalinity (as $\mathrm{CaCO}_{3}$ ) & 5 & 1 & 5 & 46 & -- & -- & -- & 51 \\
\hline Sulfate, dissolved & 6 & 0.1 & 6 & 2.9 & 3.0 & 3.4 & 3.6 & 4.0 \\
\hline Chloride, dissolved & 6 & 0.1 & 6 & 10 & 13 & 14 & 17 & 18 \\
\hline Fluoride, dissolved & 6 & 0.1 & 1 & -- & -- & -- & -- & 0.1 \\
\hline Silica, dissolved & 6 & 0.01 & 6 & 26 & 27 & 28 & 28 & 28 \\
\hline $\begin{array}{l}\text { Nitrogen, nitrite plus } \\
\text { nitrate, total (as N) }\end{array}$ & 11 & 0.1 & 0 & -- & -- & -- & -- & -- \\
\hline $\begin{array}{l}\text { Nitrogen, ammonia } \\
\text { plus organic, total (as N) }\end{array}$ & 11 & 0.2 & 7 & $<0.2$ & $\mathrm{e} 0.13$ & $\mathrm{e} 0.20$ & $\mathrm{e} 0.50$ & 0.60 \\
\hline Phosphorus, total (as P) & 11 & 0.01 & 9 & $<0.01$ & $\mathrm{e} 0.01$ & $\mathrm{e} 0.02$ & $\mathrm{e} 0.07$ & 0.14 \\
\hline \multicolumn{9}{|c|}{ During construction, November 1988 to November 1994, major ions and nutrients (mg/L) } \\
\hline Hardness, total (as $\mathrm{CaCO}_{3}$ ) & 13 & 1 & 13 & 34 & 42 & 44 & 46 & 49 \\
\hline Calcium, dissolved & 13 & 0.02 & 13 & 7.5 & 8.2 & 8.4 & 8.8 & 10 \\
\hline Magnesium, dissolved & 13 & 0.01 & 13 & 3.7 & 5.3 & 5.6 & 5.8 & 6.0 \\
\hline Sodium, dissolved & 13 & 0.2 & 13 & 11 & 12 & 12 & 12 & 13 \\
\hline Potassium, dissolved & 13 & 0.1 & 13 & 0.2 & 0.8 & 0.8 & 1.0 & 1.2 \\
\hline Alkalinity (as $\mathrm{CaCO}_{3}$ ) & 13 & 1 & 13 & 18 & 45 & 47 & 48 & 49 \\
\hline Sulfate, dissolved & 13 & 0.1 & 13 & 1.4 & 3.0 & 3.1 & 4.5 & 5.7 \\
\hline Chloride, dissolved & 13 & 0.1 & 13 & 14 & 14 & 16 & 18 & 21 \\
\hline Fluoride, dissolved & 13 & 0.1 & 4 & $<0.1$ & -- & -- & -- & 0.2 \\
\hline Silica, dissolved & 13 & 0.01 & 13 & 12 & 26 & 26 & 27 & 28 \\
\hline $\begin{array}{l}\text { Nitrogen, nitrite plus } \\
\text { nitrate, total (as N) }\end{array}$ & 17 & 0.1 & 12 & $<0.1$ & $\mathrm{e} 0.09$ & $\mathrm{e} 0.10$ & $\mathrm{e} 0.15$ & 0.24 \\
\hline $\begin{array}{l}\text { Nitrogen, nitrite plus } \\
\text { nitrate, dissolved (as N) }\end{array}$ & 4 & 0.05 & 4 & $<0.12$ & -- & -- & -- & 0.17 \\
\hline $\begin{array}{l}\text { Nitrogen, ammonia } \\
\text { plus organic, total (as } \mathrm{N} \text { ) }\end{array}$ & 25 & 0.2 & 4 & $<0.2$ & -- & -- & -- & 0.30 \\
\hline Phosphorus, total (as P) & 25 & 0.01 & 21 & $<0.01$ & $\mathrm{e} 0.01$ & $\mathrm{e} 0.02$ & $\mathrm{e} 0.03$ & 0.16 \\
\hline \multicolumn{9}{|c|}{ After construction, December 1994 to April 1998, major ions and nutrients (mg/L) } \\
\hline Hardness, total (as $\mathrm{CaCO}_{3}$ ) & 6 & 1 & 6 & 42 & 42 & 44 & 45 & 45 \\
\hline Calcium, dissolved & 6 & 0.02 & 6 & 7.9 & 8.1 & 8.4 & 8.7 & 9.2 \\
\hline Magnesium, dissolved & 6 & 0.01 & 6 & 5.3 & 5.3 & 5.5 & 5.7 & 5.7 \\
\hline Sodium, dissolved & 6 & 0.2 & 6 & 11 & 12 & 12 & 12 & 12 \\
\hline Potassium, dissolved & 6 & 0.1 & 6 & 0.7 & 0.8 & 0.8 & 0.9 & 0.9 \\
\hline Alkalinity (as $\mathrm{CaCO}_{3}$ ) & 6 & 1 & 6 & 47 & 47 & 48 & 48 & 49 \\
\hline Sulfate, dissolved & 6 & 0.1 & 6 & 3.0 & 3.2 & 3.3 & 3.6 & 3.8 \\
\hline Chloride, dissolved & 6 & 0.1 & 6 & 15 & 15 & 15 & 15 & 15 \\
\hline Fluoride, dissolved & 6 & 0.1 & 0 & -- & -- & -- & -- & -- \\
\hline Silica, dissolved & 6 & 0.01 & 6 & 26 & 26 & 26 & 27 & 27 \\
\hline $\begin{array}{l}\text { Nitrogen, nitrite plus } \\
\text { nitrate, dissolved (as N) }\end{array}$ & 12 & 0.05 & 12 & 0.06 & 0.08 & 0.09 & 0.12 & 0.13 \\
\hline $\begin{array}{l}\text { Nitrogen, ammonia } \\
\text { plus organic, total (as } \mathrm{N} \text { ) }\end{array}$ & 12 & 0.1 & 0 & -- & -- & -- & -- & -- \\
\hline Phosphorus, total (as P) & 12 & 0.05 & 10 & $<0.05$ & $\mathrm{e} 0.01$ & $\mathrm{e} 0.01$ & $\mathrm{e} 0.03$ & 0.04 \\
\hline
\end{tabular}


Table 23. Statistical summary of selected water-quality data collected at station 16275000, Haiku Stream near Heeia, Oahu, Hawaii, from March 1983 to April 1998--Continued

\begin{tabular}{|c|c|c|c|c|c|c|c|c|}
\hline Property or constituent & $\begin{array}{l}\text { Number } \\
\text { of } \\
\text { samples }\end{array}$ & $\begin{array}{c}\text { Minimum } \\
\text { detection } \\
\text { limit }\end{array}$ & $\begin{array}{l}\text { Number } \\
\text { of } \\
\text { detections }\end{array}$ & $\begin{array}{l}\text { Mini- } \\
\text { mum }\end{array}$ & \multicolumn{3}{|c|}{ Concentration percentile } & $\begin{array}{l}\text { Maxi- } \\
\text { mum }\end{array}$ \\
\hline \multicolumn{9}{|c|}{ Before construction, March 1983 to September 1988, trace metals $(\mu \mathrm{g} / \mathrm{L})$} \\
\hline Aluminum, total recoverable & e 6 & 10 & 6 & 30 & 38 & 40 & 108 & 130 \\
\hline Aluminum, dissolved & 6 & 10 & 2 & $<10$ & -- & -- & -- & 20 \\
\hline Cadmium, total recoverable & 6 & 1 & 0 & -- & -- & -- & -- & -- \\
\hline Cadmium, dissolved & 6 & 1 & 0 & -- & -- & -- & -- & -- \\
\hline Chromium, total recoverable & 6 & 10 & 3 & $<10$ & -- & -- & -- & 10 \\
\hline Chromium, dissolved & 6 & 1 & 1 & -- & -- & -- & -- & 1.0 \\
\hline Copper, total recoverable & 6 & 1 & 6 & 1.0 & 1.0 & 2.5 & 4.2 & 5.0 \\
\hline Copper, dissolved & 6 & 1 & 4 & $<4$ & -- & -- & -- & 4.0 \\
\hline Iron, total recoverable & 6 & 10 & 6 & 170 & 192 & 245 & 378 & 400 \\
\hline Iron, dissolved & 6 & 3 & 6 & 57 & 74 & 95 & 112 & 120 \\
\hline Lead, total recoverable & 6 & 1 & 2 & $<1$ & -- & -- & -- & 1.0 \\
\hline Lead, dissolved & 6 & 1 & 2 & $<1$ & -- & -- & -- & 8.0 \\
\hline Nickel, total recoverable & 6 & 1 & 4 & $<1$ & -- & -- & -- & 13 \\
\hline Nickel, dissolved & 6 & 1 & 6 & $<1$ & -- & -- & -- & 7.0 \\
\hline Zinc, total recoverable & 6 & 10 & 4 & $<10$ & -- & -- & -- & 420 \\
\hline Zinc, dissolved & 6 & 3 & 2 & $<3$ & & -- & -- & 11 \\
\hline \multicolumn{9}{|c|}{ During construction, November 1988 to November 1994 , trace metals $(\mu \mathrm{g} / \mathrm{L})$} \\
\hline Aluminum, total recoverable & e 13 & 10 & 13 & 10 & 30 & 50 & 130 & 16,000 \\
\hline Aluminum, dissolved & 13 & 10 & 8 & $<10$ & e4.3 & e10 & e50 & 50 \\
\hline Cadmium, total recoverable & 13 & 1 & 1 & -- & -- & -- & -- & 8.0 \\
\hline Cadmium, dissolved & 6 & 1 & 1 & -- & -- & -- & -- & 2.0 \\
\hline Chromium, total recoverable & e 13 & 10 & 5 & $<10$ & $\mathrm{e} 0.02$ & $\mathrm{e} 0.17$ & $\mathrm{e} 1.5$ & 55 \\
\hline Chromium, dissolved & 6 & 1 & 0 & -- & -- & -- & -- & -- \\
\hline Copper, total recoverable & 13 & 1 & 5 & $<1$ & $\mathrm{e} 0.04$ & $\mathrm{e} 0.21$ & $\mathrm{e} 2.0$ & 25 \\
\hline Copper, dissolved & 6 & 1 & 5 & $<1$ & $\mathrm{e} 0.91$ & e1.5 & $\mathrm{e} 2.0$ & 2.0 \\
\hline Iron, total recoverable & 13 & 10 & 13 & 70 & 85 & 130 & 345 & 21,000 \\
\hline Iron, dissolved & 13 & 3 & 13 & 26 & 40 & 47 & 68 & 100 \\
\hline Lead, total recoverable & 13 & 1 & 3 & $<1$ & -- & -- & -- & 4.0 \\
\hline Lead, dissolved & 6 & 1 & 1 & -- & -- & -- & -- & 1.0 \\
\hline Nickel, total recoverable & 13 & 1 & 6 & $<1$ & $\mathrm{e} 0.03$ & $\mathrm{e} 0.20$ & $\mathrm{e} 2.0$ & 43 \\
\hline Nickel, dissolved & 13 & 1 & 3 & $<1$ & -- & -- & -- & 1.0 \\
\hline Zinc, total recoverable & 13 & 10 & 3 & $<10$ & -- & -- & -- & 40 \\
\hline Zinc, dissolved & 6 & 3 & 5 & $<3$ & e3.6 & $\mathrm{e} 4.5$ & e8.0 & 8.0 \\
\hline \multicolumn{9}{|c|}{ After construction, December 1994 to April 1998, trace metals $(\mu \mathrm{g} / \mathrm{L})$} \\
\hline Aluminum, total recoverable & e 6 & 10 & 6 & 10 & 25 & 30 & 32 & 40 \\
\hline Aluminum, dissolved & 6 & 10 & 4 & $<10$ & -- & -- & -- & 20 \\
\hline Cadmium, total recoverable & 6 & 1 & 0 & -- & -- & -- & -- & -- \\
\hline Cadmium, dissolved & 0 & 1 & -- & -- & -- & -- & -- & -- \\
\hline Chromium, total recoverable & 6 & 1 & 1 & -- & -- & -- & -- & 1 \\
\hline Chromium, dissolved & 0 & 1 & -- & -- & -- & -- & -- & -- \\
\hline Copper, total recoverable & 6 & 1 & 0 & -- & -- & -- & -- & -- \\
\hline Copper, dissolved & 0 & 1 & -- & -- & -- & -- & -- & -- \\
\hline Iron, total recoverable & 6 & 10 & 6 & 50 & 58 & 95 & 152 & 160 \\
\hline Iron, dissolved & 6 & 3 & 6 & 16 & 28 & 41 & 76 & 84 \\
\hline Lead, total recoverable & 6 & 1 & 0 & -- & -- & -- & -- & -- \\
\hline Lead, dissolved & 0 & 1 & -- & -- & -- & -- & -- & -- \\
\hline Nickel, total recoverable & 6 & 1 & 0 & -- & -- & -- & -- & -- \\
\hline Nickel, dissolved & 6 & 1 & 0 & -- & -- & -- & -- & -- \\
\hline Zinc, total recoverable & 6 & 10 & 0 & -- & -- & -- & -- & -- \\
\hline Zinc, dissolved & 0 & 20 & -- & -- & -- & -- & -- & -- \\
\hline
\end{tabular}


Table 24. Statistical summary of selected water-quality data collected at station 212335157482603, Waimaluhia Reservoir cross-section 1-3 (composite) near Kaneohe, Oahu, Hawaii, from May 1983 to January 1998

[inst., instantaneous; $\mu \mathrm{S} / \mathrm{cm}$ at $25^{\circ} \mathrm{C}$, microsiemens per centimeter at 25 degrees Celsius; $\mathrm{mg} / \mathrm{L}$, milligram per liter; $\mu \mathrm{g} / \mathrm{L}$, microgram per liter; <, less than; e, estimated using log-probability regression; $0.7 \mu \mathrm{m}-\mathrm{MF}, 0.7$ micron membrane filter; cols./100 mL, colonies per 100 milliliters; $\mathrm{ft}^{3} / \mathrm{s}$, cubic foot per second; ${ }^{\circ} \mathrm{C}$, degrees Celsius; $\mathrm{NTU}$, nephelometric turbidity unit; --, no data or not computed; Range of dates are the time periods of water-quality data collection during the specified construction activity]

\begin{tabular}{|c|c|c|c|c|c|c|c|c|}
\hline \multirow[b]{2}{*}{ Property or constituent } & \multirow{2}{*}{$\begin{array}{l}\text { Number } \\
\text { of } \\
\text { samples }\end{array}$} & \multirow{2}{*}{$\begin{array}{l}\text { Minimum } \\
\text { detection } \\
\text { limit }\end{array}$} & \multirow{2}{*}{$\begin{array}{l}\text { Number } \\
\text { of } \\
\text { detections }\end{array}$} & \multirow{2}{*}{$\begin{array}{l}\text { Mini- } \\
\text { mum }\end{array}$} & \multicolumn{3}{|c|}{ Concentration percentile } & \multirow{2}{*}{$\begin{array}{l}\text { Maxi- } \\
\text { mum }\end{array}$} \\
\hline & & & & & 25 & 50 & 75 & \\
\hline \multicolumn{9}{|c|}{ During construction, May 1983 to June 1992, physical, biological, and organic } \\
\hline \multicolumn{9}{|c|}{ Specific conductance } \\
\hline$\left(\mu \mathrm{S} / \mathrm{cm}\right.$ at $\left.25^{\circ} \mathrm{C}\right)$ & 274 & 1 & 274 & 147 & 182 & 186 & 191 & 210 \\
\hline $\mathrm{pH}$ (units) & 271 & 0.1 & 271 & 6.4 & 7.2 & 7.4 & 7.6 & 8.6 \\
\hline Temperature, water $\left({ }^{\circ} \mathrm{C}\right)$ & 274 & 0.5 & 274 & 20.7 & 23.1 & 25.0 & 25.9 & 28.8 \\
\hline Turbidity (NTU) & 113 & 0.1 & 113 & 0.4 & 1.2 & 1.7 & 2.3 & 170 \\
\hline Oxygen dissolved (mg/L) & 273 & 0.1 & 273 & 2.6 & 6.0 & 7.0 & 7.6 & 9.6 \\
\hline $\begin{array}{l}\text { Fecal coliform } 0.7 \mu \mathrm{m}-\mathrm{MF} \\
(\text { cols. } / 100 \mathrm{~mL})\end{array}$ & 72 & 0 & 71 & 1.0 & 7.0 & 20 & 64 & 640 \\
\hline $\begin{array}{l}\text { Solids, residue at } 105^{\circ} \mathrm{C}, \\
\text { suspended }(\mathrm{mg} / \mathrm{L})\end{array}$ & 78 & 1 & 60 & $<1$ & $\mathrm{e} 1.0$ & $\mathrm{e} 3.0$ & $\mathrm{e} 7.0$ & 23 \\
\hline Carbon, organic, total $(\mathrm{mg} / \mathrm{L})$ & L) 13 & 0.1 & 13 & 1.4 & 1.6 & 2.0 & 2.5 & 4.7 \\
\hline Oil and grease, total $(\mathrm{mg} / \mathrm{L})$ & 12 & 1 & 1 & -- & -- & -- & -- & 1 \\
\hline \multicolumn{9}{|c|}{ After construction, August 1992 to January 1998, physical, biological, and organic } \\
\hline $\begin{array}{l}\text { Specific conductance } \\
\left(\mu \mathrm{S} / \mathrm{cm} \text { at } 25^{\circ} \mathrm{C}\right)\end{array}$ & 106 & 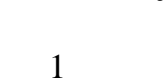 & 106 & 152 & 186 & 191 & 196 & 214 \\
\hline pH (units) & 106 & 0.1 & 106 & 6.9 & 7.2 & 7.4 & 7.6 & 7.8 \\
\hline Temperature, water $\left({ }^{\circ} \mathrm{C}\right)$ & 106 & 0.5 & 106 & 20.5 & 21.5 & 24.0 & 25.9 & 28.2 \\
\hline Turbidity (NTU) & 55 & 0.1 & 55 & 0.3 & 1.2 & 1.5 & 5.0 & 27 \\
\hline Oxygen dissolved (mg/L) & 106 & 0.1 & 106 & 2.1 & 6.0 & 6.6 & 7.1 & 8.7 \\
\hline $\begin{array}{l}\text { Fecal coliform } 0.7 \mu \mathrm{m}-\mathrm{MF} \\
(\text { cols. } / 100 \mathrm{~mL} \text { ) }\end{array}$ & 22 & 0 & 22 & 4 & 14 & 40 & 105 & 1,200 \\
\hline $\begin{array}{l}\text { Solids, residue at } 105^{\circ} \mathrm{C}, \\
\text { suspended }(\mathrm{mg} / \mathrm{L})\end{array}$ & 29 & 1 & 24 & $<1$ & e0.6 & $\mathrm{e} 3.0$ & $\mathrm{e} 5.5$ & 10 \\
\hline Carbon, organic, total $(\mathrm{mg} / \mathrm{L})$ & L) 6 & 0.1 & 6 & 1.7 & 1.8 & 2.0 & 2.0 & 2.2 \\
\hline Oil and grease, total $(\mathrm{mg} / \mathrm{L})$ & 6 & 1 & 2 & $<1$ & -- & -- & -- & 3 \\
\hline
\end{tabular}


Table 24. Statistical summary of selected water-quality data collected at station 212335157482603, Waimaluhia Reservoir cross-section 1-3 (composite) near Kaneohe, Oahu, Hawaii, from May 1983 to January 1998--Continued

\begin{tabular}{|c|c|c|c|c|c|c|c|c|}
\hline Property or constituent & $\begin{array}{l}\text { Number } \\
\text { of } \\
\text { samples }\end{array}$ & $\begin{array}{l}\text { Minimum } \\
\text { detection } \\
\text { limit }\end{array}$ & $\begin{array}{l}\text { Number } \\
\text { of } \\
\text { detections }\end{array}$ & $\begin{array}{l}\text { Mini- } \\
\text { mum }\end{array}$ & $\frac{\text { Conce }}{25}$ & $\frac{\text { ration } p}{50}$ & $\frac{\text { centile }}{75}$ & $\begin{array}{l}\text { Maxi- } \\
\text { mum }\end{array}$ \\
\hline \multicolumn{9}{|c|}{ During construction, May 1983 to June 1992, major ions and nutrients $(\mathrm{mg} / \mathrm{L})$} \\
\hline Hardness, total (as $\mathrm{CaCO}_{3}$ ) & 13 & 1 & 13 & 52 & 56 & 57 & 60 & 52 \\
\hline Calcium, dissolved & 13 & 0.02 & 13 & 7.9 & 8.5 & 8.9 & 9.8 & 11 \\
\hline Magnesium, dissolved & 13 & 0.01 & 13 & 7.5 & 7.9 & 8.5 & 8.8 & 7.5 \\
\hline Sodium, dissolved & 13 & 0.2 & 13 & 14 & 15 & 15 & 16 & 17 \\
\hline Potassium, dissolved & 13 & 0.1 & 13 & 0.8 & 1.0 & 1.0 & 1.2 & 1.4 \\
\hline Alkalinity (as $\mathrm{CaCO}_{3}$ ) & 13 & 1 & 12 & $<1$ & e50 & e53 & e54 & 63 \\
\hline Sulfate, dissolved & 13 & 0.1 & 13 & 6.7 & 7.0 & 8.2 & 9.0 & 11 \\
\hline Chloride, dissolved & 13 & 0.1 & 13 & 17 & 18 & 19 & 22 & 22 \\
\hline Fluoride, dissolved & 13 & 0.1 & 5 & $<0.1$ & $\mathrm{e} 0.1$ & $\mathrm{e} 0.1$ & $\mathrm{e} 0.1$ & 0.1 \\
\hline Silica, dissolved & 13 & 0.01 & 13 & 18 & 18 & 20 & 21 & 20 \\
\hline $\begin{array}{l}\text { Nitrogen, nitrite plus } \\
\text { nitrate, total (as } \mathrm{N})\end{array}$ & 73 & 0.05 & 73 & 0.10 & 0.20 & 0.30 & 0.40 & 0.50 \\
\hline $\begin{array}{l}\text { Nitrogen, nitrite plus } \\
\text { nitrate, dissolved (as N) }\end{array}$ & 0 & 0.05 & -- & -- & -- & -- & -- & -- \\
\hline $\begin{array}{l}\text { Nitrogen, ammonia } \\
\text { plus organic, total (as N) }\end{array}$ & 75 & 0.2 & 69 & $<0.2$ & $\mathrm{e} 0.30$ & $e 0.40$ & $\mathrm{e} 0.50$ & 1.1 \\
\hline Phosphorus, total (as P) & 75 & 0.01 & 66 & $<0.01$ & $\mathrm{e} 0.01$ & $\mathrm{e} 0.02$ & $\mathrm{e} 0.03$ & 0.11 \\
\hline \multicolumn{9}{|c|}{ After construction, August 1992 to January 1998, major ions and nutrients (mg/L) } \\
\hline Hardness, total (as $\mathrm{CaCO}_{3}$ ) & 7 & 1 & 7 & 43 & 53 & 56 & 58 & 58 \\
\hline Calcium, dissolved & 7 & 0.02 & 7 & 6.0 & 7.6 & 8.4 & 8.8 & 11 \\
\hline Magnesium, dissolved & 7 & 0.01 & 7 & 6.9 & 7.3 & 8.3 & 8.5 & 9.0 \\
\hline Sodium, dissolved & 7 & 0.2 & 7 & 12 & 13 & 15 & 16 & 17 \\
\hline Potassium, dissolved & 7 & 0.1 & 7 & 0.2 & 0.9 & 1.2 & 1.2 & 1.9 \\
\hline Alkalinity $\left(\right.$ as $\mathrm{CaCO}_{3}$ ) & 7 & 1 & 7 & 52 & 55 & 56 & 58 & 60 \\
\hline Sulfate, dissolved & 7 & 0.1 & 7 & 5.1 & 5.8 & 6.4 & 7.8 & 8.6 \\
\hline Chloride, dissolved & 7 & 0.1 & 7 & 15 & 17 & 20 & 20 & 23 \\
\hline Fluoride, dissolved & 7 & 0.1 & 2 & $<0.1$ & -- & -- & -- & 0.1 \\
\hline Silica, dissolved & 7 & 0.01 & 7 & 16 & 16 & 19 & 20 & 16 \\
\hline $\begin{array}{l}\text { Nitrogen, nitrite plus } \\
\text { nitrate, dissolved (as N) }\end{array}$ & 16 & 0.05 & 16 & 0.30 & 0.34 & 0.39 & 0.47 & 0.55 \\
\hline $\begin{array}{l}\text { Nitrogen, ammonia } \\
\text { plus organic, total (as N) }\end{array}$ & 22 & 0.1 & 3 & $<0.1$ & -- & -- & -- & 0.30 \\
\hline Phosphorus, total (as P) & 22 & 0.05 & 13 & $<0.05$ & $\mathrm{e} 0.01$ & $\mathrm{e} 0.01$ & $\mathrm{e} 0.02$ & 0.03 \\
\hline
\end{tabular}


Table 24. Statistical summary of selected water-quality data collected at station 212335157482603, Waimaluhia Reservoir cross-section 1-3 (composite) near Kaneohe, Oahu, Hawaii, from May 1983 to January 1998--Continued

\begin{tabular}{|c|c|c|c|c|c|c|c|c|}
\hline Property or constituent & $\begin{array}{l}\text { Number } \\
\text { of } \\
\text { samples }\end{array}$ & $\begin{array}{l}\text { Minimum } \\
\text { detection } \\
\text { limit }\end{array}$ & $\begin{array}{l}\text { Number } \\
\text { of } \\
\text { detections }\end{array}$ & $\begin{array}{l}\text { Mini- } \\
\text { mum }\end{array}$ & $\frac{\text { Conc }}{25}$ & $\frac{\text { tration } p}{50}$ & $\frac{\text { centile }}{75}$ & $\begin{array}{l}\text { Maxi- } \\
\text { mum }\end{array}$ \\
\hline \multicolumn{9}{|c|}{ During construction, May 1983 to June 1992, trace metals $(\mu \mathrm{g} / \mathrm{L})$} \\
\hline Aluminum, total recoverable & e 13 & 10 & 13 & 52 & 56 & 57 & 60 & 64 \\
\hline Aluminum, dissolved & 13 & 10 & 8 & $<10$ & e2.9 & $\mathrm{e} 5.2$ & e15 & 40 \\
\hline Cadmium, total recoverable & 13 & 1 & 0 & -- & -- & -- & -- & -- \\
\hline Cadmium, dissolved & 12 & 1 & 2 & $<1$ & -- & -- & -- & 2.0 \\
\hline Chromium, total recoverable & e 13 & 1 & 6 & $<1$ & $\mathrm{e} 0.7$ & $\mathrm{e} 1.0$ & $\mathrm{e} 2.0$ & 3.0 \\
\hline Chromium, dissolved & 12 & 1 & 1 & -- & -- & -- & -- & 1.0 \\
\hline Copper, total recoverable & 13 & 1 & 13 & 2.0 & 2.0 & 4.0 & 6.0 & 8 \\
\hline Copper, dissolved & 12 & 1 & 10 & $<1$ & $\mathrm{e} 1.0$ & $\mathrm{e} 1.5$ & e2.8 & 4.0 \\
\hline Iron, total recoverable & 13 & 10 & 13 & 160 & 195 & 330 & 435 & 600 \\
\hline Iron, dissolved & 13 & 3 & 12 & $<3$ & e10 & e14 & e41 & 41 \\
\hline Lead, total recoverable & 13 & 1 & 8 & $<1$ & $\mathrm{e} 1.0$ & $\mathrm{e} 2.0$ & $\mathrm{e} 3.0$ & 6.0 \\
\hline Lead, dissolved & 12 & 1 & 3 & 1 & -- & -- & -- & 15 \\
\hline Nickel, total recoverable & 13 & 1 & 12 & $<1$ & & & & \\
\hline Nickel, dissolved & 13 & 1 & 6 & $<1$ & $\mathrm{e} 0.7$ & e1.0 & e1.0 & 2.0 \\
\hline Zinc, total recoverable & 13 & 10 & 5 & $<10$ & $\mathrm{e} 1.1$ & e3.4 & e15 & 60 \\
\hline Zinc, dissolved & 12 & 3 & 7 & $<3$ & e3.9 & $\mathrm{e} 4.8$ & e5.0 & 6.0 \\
\hline \multicolumn{9}{|c|}{ After construction, August 1992 to January 1998, trace metals $(\mu \mathrm{g} / \mathrm{L})$} \\
\hline Aluminum, total recoverable & 7 & 10 & 7 & 60 & 120 & 140 & 210 & 270 \\
\hline Aluminum, dissolved & 7 & 10 & 5 & $<10$ & e7.1 & e20 & e20 & 40 \\
\hline Cadmium, total recoverable & 7 & 1 & 0 & -- & -- & -- & -- & -- \\
\hline Cadmium, dissolved & 0 & 1 & -- & -- & -- & -- & -- & -- \\
\hline Chromium, total recoverable & 7 & 1 & 1 & -- & -- & -- & -- & 2 \\
\hline Chromium, dissolved & 0 & 1 & -- & -- & -- & -- & -- & -- \\
\hline Copper, total recoverable & 7 & 1 & 5 & $<1$ & $\mathrm{e} 0.5$ & e1.0 & e2.0 & 3.0 \\
\hline Copper, dissolved & 0 & 1 & -- & -- & -- & -- & -- & -- \\
\hline Iron, total recoverable & 7 & 10 & 7 & 120 & 310 & 350 & 430 & 480 \\
\hline Iron, dissolved & 7 & 3 & 7 & 19 & 19 & 21 & 74 & 74 \\
\hline Lead, total recoverable & 7 & 1 & 2 & $<1$ & -- & -- & -- & 2 \\
\hline Lead, dissolved & 0 & 1 & -- & -- & -- & -- & -- & -- \\
\hline Nickel, total recoverable & 7 & 1 & 6 & -- & -- & -- & -- & -- \\
\hline Nickel, dissolved & 7 & 1 & 0 & -- & -- & -- & -- & -- \\
\hline Zinc, total recoverable & 7 & 10 & 1 & -- & -- & -- & -- & 10 \\
\hline Zinc, dissolved & 0 & 20 & -- & -- & -- & -- & -- & -- \\
\hline
\end{tabular}


Table 25. Statistical summary of selected water-quality data collected at station 212335157482603 , Waimaluhia Reservoir cross-section 1-3 at $1 \mathrm{ft}$ depth near Kaneohe, Oahu, Hawaii, from May 1983 to January 1998

[inst., instantaneous; $\mu \mathrm{S} / \mathrm{cm}$ at $25^{\circ} \mathrm{C}$, microsiemens per centimeter at 25 degrees Celsius; $\mathrm{mg} / \mathrm{L}$, milligram per liter; $\mu \mathrm{g} / \mathrm{L}$, microgram per liter; <, less than; e, estimated using log-probability regression; $0.7 \mu \mathrm{m}-\mathrm{MF}, 0.7$ micron membrane filter; cols. $/ 100 \mathrm{~mL}$, colonies per 100 milliliters; $\mathrm{ft}^{3} / \mathrm{s}$, cubic foot per second; ${ }^{\circ} \mathrm{C}$, degrees Celsius; $\mathrm{NTU}$, nephelometric turbidity unit; --, no data or not computed; Range of dates are the time periods of water-quality data collection during the specified construction activity]

\begin{tabular}{|c|c|c|c|c|c|c|c|c|}
\hline Property or constituent & $\begin{array}{l}\text { Number } \\
\text { of } \\
\text { samples }\end{array}$ & $\begin{array}{l}\text { Minimum } \\
\text { detection } \\
\text { limit }\end{array}$ & $\begin{array}{l}\text { Number } \\
\text { of } \\
\text { detections }\end{array}$ & $\begin{array}{l}\text { Mini- } \\
\text { mum }\end{array}$ & $\frac{\text { Conce }}{25}$ & $\frac{\text { ration pe }}{50}$ & $\frac{\text { entile }}{75}$ & $\begin{array}{l}\text { Maxi- } \\
\text { mum }\end{array}$ \\
\hline \multicolumn{9}{|c|}{ During construction, May 1983 to June 1992, physical, biological, and nutrients } \\
\hline \multicolumn{9}{|c|}{ Specific conductance } \\
\hline$\left(\mu \mathrm{S} / \mathrm{cm}\right.$ at $\left.25^{\circ} \mathrm{C}\right)$ & 38 & 1 & 38 & 156 & 180 & 184 & 192 & 208 \\
\hline $\mathrm{pH}$ (units) & 37 & 0.1 & 37 & 6.9 & 7.4 & 7.6 & 7.8 & 8.6 \\
\hline Temperature, water $\left({ }^{\circ} \mathrm{C}\right)$ & 38 & 0.5 & 38 & 21.4 & 23.8 & 25.5 & 26.4 & 28.8 \\
\hline Turbidity (NTU) & 34 & 0.1 & 34 & 0.5 & 0.9 & 1.2 & 1.7 & 3.8 \\
\hline Oxygen dissolved (mg/L) & 38 & 0.1 & 38 & 6.1 & 7.3 & 7.6 & 8.1 & 9.5 \\
\hline $\begin{array}{l}\text { Fecal coliform } 0.7 \mu \mathrm{m}-\mathrm{MF} \\
(\text { cols. } / 100 \mathrm{~mL})\end{array}$ & 24 & 0 & 24 & 1 & 3 & 10 & 38 & 190 \\
\hline $\begin{array}{l}\text { Solids, residue at } 105^{\circ} \mathrm{C}, \\
\text { suspended }(\mathrm{mg} / \mathrm{L})\end{array}$ & 26 & 1 & 20 & $<1$ & $\mathrm{e} 0.64$ & $\mathrm{e} 2.0$ & e6.2 & 21 \\
\hline $\begin{array}{l}\text { Nitrogen, nitrite plus } \\
\text { nitrate, total (as N) }\end{array}$ & 25 & 0.05 & 25 & 0.10 & 0.20 & 0.30 & 0.40 & 0.44 \\
\hline $\begin{array}{l}\text { Nitrogen, nitrite plus } \\
\text { nitrate, dissolved (as N) }\end{array}$ & 0 & 0.05 & -- & -- & -- & -- & -- & -- \\
\hline $\begin{array}{l}\text { Nitrogen, ammonia } \\
\text { plus organic, total (as N) }\end{array}$ & 25 & 0.2 & 23 & $<0.2$ & $\mathrm{e} 0.20$ & $\mathrm{e} 0.40$ & $\mathrm{e} 0.45$ & 1.1 \\
\hline Phosphorus, total (as P) & 25 & 0.01 & 20 & $<0.01$ & $\mathrm{e} 0.01$ & $\mathrm{e} 0.02$ & $\mathrm{e} 0.02$ & 0.08 \\
\hline \multicolumn{9}{|c|}{ After construction, August 1992 to January 1998, physical, biological, and nutrients } \\
\hline $\begin{array}{l}\text { Specific conductance } \\
\left(\mu \mathrm{S} / \mathrm{cm} \text { at } 25^{\circ} \mathrm{C}\right)\end{array}$ & 15 & 1 & 15 & 152 & 182 & 188 & 194 & 200 \\
\hline $\mathrm{pH}$ (units) & 15 & 0.1 & 15 & 6.9 & 7.2 & 7.4 & 7.6 & 7.8 \\
\hline Temperature, water $\left({ }^{\circ} \mathrm{C}\right)$ & 15 & 0.5 & 15 & 20.9 & 21.6 & 24.0 & 26.0 & 27.0 \\
\hline Turbidity (NTU) & 15 & 0.1 & 15 & 0.3 & 1.0 & 1.5 & 5.0 & 9.4 \\
\hline Oxygen dissolved (mg/L) & 15 & 0.1 & 15 & 6.3 & 6.7 & 6.9 & 7.2 & 8.3 \\
\hline $\begin{array}{l}\text { Fecal coliform } 0.7 \mu \mathrm{m}-\mathrm{MF} \\
(\text { cols. } / 100 \mathrm{~mL})\end{array}$ & 7 & 0 & 7 & 5 & 13 & 30 & 100 & 720 \\
\hline $\begin{array}{l}\text { Solids, residue at } 105^{\circ} \mathrm{C}, \\
\text { suspended }(\mathrm{mg} / \mathrm{L})\end{array}$ & 8 & 1 & 7 & $<1$ & $\mathrm{e} 0.57$ & $\mathrm{e} 1.5$ & $\mathrm{e} 3.5$ & 6 \\
\hline $\begin{array}{l}\text { Nitrogen, nitrite plus } \\
\text { nitrate, dissolved (as N) }\end{array}$ & 4 & 0.05 & 4 & 0.34 & -- & 0.42 & -- & 0.49 \\
\hline $\begin{array}{l}\text { Nitrogen, ammonia } \\
\text { plus organic, total (as N) }\end{array}$ & 6 & 0.1 & 0 & -- & -- & -- & -- & -- \\
\hline Phosphorus, total (as P) & 6 & 0.05 & 3 & $<0.05$ & -- & -- & -- & 0.01 \\
\hline
\end{tabular}


Table 26. Statistical summary of selected water-quality data collected at station 212335157482603 , Waimaluhia Reservoir cross-section 1-3 at $7 \mathrm{ft}$ depth near Kaneohe, Oahu, Hawaii, from May 1983 to January 1998

[inst., instantaneous; $\mu \mathrm{S} / \mathrm{cm}$ at $25^{\circ} \mathrm{C}$, microsiemens per centimeter at 25 degrees Celsius; $\mathrm{mg} / \mathrm{L}$, milligram per liter; $\mu \mathrm{g} / \mathrm{L}$, microgram per liter; <, less than; e, estimated using log-probability regression; $0.7 \mu \mathrm{m}-\mathrm{MF}, 0.7$ micron membrane filter; cols./100 mL, colonies per 100 milliliters; $\mathrm{ft}^{3} / \mathrm{s}$, cubic foot per second; ${ }^{\circ} \mathrm{C}$, degrees Celsius; NTU, nephelometric turbidity unit; --, no data or not computed; Range of dates are the time periods of water-quality data collection during the specified construction activity]

\begin{tabular}{|c|c|c|c|c|c|c|c|c|}
\hline Property or constituent & $\begin{array}{l}\text { Number } \\
\text { of } \\
\text { samples }\end{array}$ & $\begin{array}{l}\text { Minimum } \\
\text { detection } \\
\text { limit }\end{array}$ & $\begin{array}{l}\text { Number } \\
\text { of } \\
\text { detections }\end{array}$ & $\begin{array}{l}\text { Mini- } \\
\text { mum }\end{array}$ & $\frac{\text { Conce }}{25}$ & $\frac{\text { tration pe }}{50}$ & $\frac{\text { centile }}{75}$ & $\begin{array}{l}\text { Maxi- } \\
\text { mum }\end{array}$ \\
\hline \multicolumn{9}{|c|}{ During construction, May 1983 to June 1992, physical, biological, and nutrients } \\
\hline $\begin{array}{l}\text { Specific conductance } \\
\left(\mathrm{uS} / \mathrm{cm} \text { at } 25^{\circ} \mathrm{C}\right)\end{array}$ & 38 & 1 & 38 & 155 & 181 & 186 & 191 & 208 \\
\hline $\mathrm{pH}$ (units) & 37 & 0.1 & 37 & 6.9 & 7.3 & 7.4 & 7.6 & 8.6 \\
\hline Temperature, water $\left({ }^{\circ} \mathrm{C}\right)$ & 38 & 0.5 & 38 & 21.1 & 23.6 & 25.0 & 26.0 & 27.6 \\
\hline Turbidity (NTU) & 33 & 0.1 & 33 & 0.4 & 1.2 & 1.3 & 2.0 & 5.9 \\
\hline Oxygen dissolved (mg/L) & 38 & 0.1 & 38 & 4.3 & 6.0 & 7.0 & 7.4 & 8.4 \\
\hline $\begin{array}{l}\text { Fecal coliform } 0.7 \mu \mathrm{m}-\mathrm{MF} \\
(\text { cols. } / 100 \mathrm{~mL})\end{array}$ & 23 & 0 & 23 & 1 & 8 & 17 & 47 & 160 \\
\hline $\begin{array}{l}\text { Solids, residue at } 105^{\circ} \mathrm{C}, \\
\text { suspended }(\mathrm{mg} / \mathrm{L})\end{array}$ & 25 & 1 & 20 & $<1$ & $\mathrm{e} 1.0$ & e3.0 & e5.0 & 16 \\
\hline $\begin{array}{l}\text { Nitrogen, nitrite plus } \\
\text { nitrate, total (as N) }\end{array}$ & 24 & 0.05 & 23 & 0.10 & 0.20 & 0.30 & 0.40 & 0.50 \\
\hline $\begin{array}{l}\text { Nitrogen, nitrite plus } \\
\text { nitrate, dissolved (as N) }\end{array}$ & 0 & 0.05 & -- & -- & -- & -- & -- & -- \\
\hline $\begin{array}{l}\text { Nitrogen, ammonia } \\
\text { plus organic, total (as N) }\end{array}$ & 24 & 0.2 & 22 & $<0.2$ & $\mathrm{e} 0.30$ & $\mathrm{e} 0.40$ & $\mathrm{e} 0.50$ & 1.0 \\
\hline Phosphorus, total (as P) & 24 & 0.01 & 21 & $<0.01$ & $\mathrm{e} 0.01$ & $\mathrm{e} 0.02$ & $\mathrm{e} 0.02$ & 0.04 \\
\hline \multicolumn{9}{|c|}{ After construction, August 1992 to January 1998, physical, biological, and nutrients } \\
\hline $\begin{array}{l}\text { Specific conductance } \\
\left(\mu \mathrm{S} / \mathrm{cm} \text { at } 25^{\circ} \mathrm{C}\right)\end{array}$ & 16 & 1 & 16 & 156 & 184 & 189 & 194 & 202 \\
\hline $\mathrm{pH}$ (units) & 16 & 0.1 & 16 & 7.1 & 7.2 & 7.4 & 7.6 & 7.8 \\
\hline Temperature, water $\left({ }^{\circ} \mathrm{C}\right)$ & 16 & 0.5 & 16 & 20.9 & 21.6 & 23.8 & 25.9 & 27.2 \\
\hline Turbidity (NTU) & 16 & 0.1 & 16 & 0.4 & 1.1 & 1.3 & 2.2 & 9.8 \\
\hline Oxygen dissolved (mg/L) & 16 & 0.1 & 16 & 4.9 & 6.0 & 6.6 & 7.1 & 8.5 \\
\hline $\begin{array}{l}\text { Fecal coliform } 0.7 \mu \mathrm{m}-\mathrm{MF} \\
(\text { cols. } / 100 \mathrm{~mL})\end{array}$ & 7 & 0 & 7 & 4 & 14 & 38 & 58 & 620 \\
\hline $\begin{array}{l}\text { Solids, residue at } 105^{\circ} \mathrm{C}, \\
\text { suspended (mg/L) }\end{array}$ & 9 & 1 & 7 & $<1$ & e1.9 & $\mathrm{e} 4.0$ & $\mathrm{e} 4.0$ & 7.0 \\
\hline $\begin{array}{l}\text { Nitrogen, nitrite plus } \\
\text { nitrate, dissolved (as N) }\end{array}$ & 5 & 0.05 & 5 & 0.31 & -- & 0.39 & -- & 0.54 \\
\hline $\begin{array}{l}\text { Nitrogen, ammonia } \\
\text { plus organic, total (as N) }\end{array}$ & 7 & 0.1 & 2 & $<0.1$ & -- & -- & -- & 0.30 \\
\hline Phosphorus, total (as P) & 7 & 0.05 & 3 & $<0.05$ & -- & -- & -- & 0.20 \\
\hline
\end{tabular}


Table 27. Statistical summary of selected water-quality data collected at station 212335157482603, Waimaluhia Reservoir cross-section 1-3 at $11 \mathrm{ft}$ depth near Kaneohe, Oahu, Hawaii, from May 1983 to January 1998

[inst., instantaneous; $\mu \mathrm{S} / \mathrm{cm}$ at $25^{\circ} \mathrm{C}$, microsiemens per centimeter at 25 degrees Celsius; $\mathrm{mg} / \mathrm{L}$, milligram per liter; $\mu \mathrm{g} / \mathrm{L}$, microgram per liter; <, less than; e, estimated using log-probability regression; $0.7 \mu \mathrm{m}-\mathrm{MF}, 0.7$ micron membrane filter; cols./100 mL, colonies per 100 milliliters; $\mathrm{ft}^{3} / \mathrm{s}$, cubic foot per second; ${ }^{\circ} \mathrm{C}$, degrees Celsius; NTU, nephelometric turbidity unit; --, no data or not computed; Range of dates are the time periods of water-quality data collection during the specified construction activity]

\begin{tabular}{|c|c|c|c|c|c|c|c|c|}
\hline \multirow[b]{2}{*}{ Property or constituent } & \multirow{2}{*}{$\begin{array}{l}\text { Number } \\
\text { of } \\
\text { samples }\end{array}$} & \multirow{2}{*}{$\begin{array}{l}\text { Minimum } \\
\text { detection } \\
\text { limit }\end{array}$} & \multirow{2}{*}{$\begin{array}{l}\text { Number } \\
\text { of } \\
\text { detections }\end{array}$} & \multirow{2}{*}{$\begin{array}{l}\text { Mini- } \\
\text { mum }\end{array}$} & \multicolumn{3}{|c|}{ Concentration percentile } & \multirow{2}{*}{$\begin{array}{l}\text { Maxi- } \\
\text { mum }\end{array}$} \\
\hline & & & & & 25 & 50 & 75 & \\
\hline \multicolumn{9}{|c|}{ During construction, May 1983 to June 1992, physical, biological, and nutrients } \\
\hline Specific conductance & & & & & & & & \\
\hline$\left(\mu \mathrm{S} / \mathrm{cm}\right.$ at $\left.25^{\circ} \mathrm{C}\right)$ & 35 & 1 & 35 & 165 & 186 & 189 & 194 & 207 \\
\hline $\mathrm{pH}$ (units) & 34 & 0.1 & 34 & 6.6 & 7.1 & 7.2 & 7.3 & 8.5 \\
\hline Temperature, water $\left({ }^{\circ} \mathrm{C}\right)$ & 35 & 0.5 & 35 & 20.9 & 23.0 & 24.2 & 25.0 & 26.5 \\
\hline Turbidity (NTU) & 20 & 0.1 & 20 & 1.0 & 1.8 & 2.2 & 3.8 & 25 \\
\hline Oxygen dissolved (mg/L) & 35 & 0.1 & 35 & 3.5 & 4.8 & 5.6 & 6.6 & 7.6 \\
\hline Fecal coliform $0.7 \mu \mathrm{m}-\mathrm{MF}$ & 14 & 0 & 14 & 10 & 26 & 72 & 110 & 640 \\
\hline Solids, residue at $105^{\circ} \mathrm{C}$, & 14 & 0 & 13 & 10 & e? 0 & e6 0 & 110 & 640 \\
\hline $\begin{array}{l}\text { Nitrogen, nitrite plus } \\
\text { nitrate, total (as N) }\end{array}$ & 15 & 0.05 & 15 & 0.30 & 0.30 & 0.40 & 0.40 & 0.49 \\
\hline $\begin{array}{l}\text { Nitrogen, nitrite plus } \\
\text { nitrate, dissolved (as N) }\end{array}$ & 0 & 0.05 & -- & -- & -- & -- & -- & -- \\
\hline $\begin{array}{l}\text { Nitrogen, ammonia } \\
\text { plus organic, total (as N) }\end{array}$ & 15 & 0.2 & 15 & 0.20 & 0.20 & 0.30 & 0.60 & 1.1 \\
\hline Phosphorus, total (as P) & 15 & 0.01 & 14 & $<0.01$ & $\mathrm{e} 0.01$ & $\mathrm{e} 0.02$ & $\mathrm{e} 0.04$ & 0.11 \\
\hline \multicolumn{9}{|c|}{ After construction, August 1992 to January 1998, physical, biological, and nutrients } \\
\hline $\begin{array}{l}\text { Specific conductance } \\
\left(\mu \mathrm{S} / \mathrm{cm} \text { at } 25^{\circ} \mathrm{C}\right)\end{array}$ & 16 & (- & 16 & 156 & 189 & 195 & 204 & 214 \\
\hline $\mathrm{pH}$ (units) & 16 & 0.1 & 16 & 7.1 & 7.2 & 7.3 & 7.3 & 7.7 \\
\hline Temperature, water $\left({ }^{\circ} \mathrm{C}\right)$ & 16 & 0.5 & 16 & 20.5 & 21.6 & 23.2 & 24.9 & 26.0 \\
\hline Turbidity (NTU) & 16 & 0.1 & 16 & 1.0 & 1.2 & 2.5 & 6.4 & 27.0 \\
\hline Oxygen dissolved (mg/L) & 16 & 0.1 & 16 & 4.2 & 5.2 & 5.6 & 6.0 & 8.7 \\
\hline $\begin{array}{l}\text { Fecal coliform } 0.7 \mu \mathrm{m}-\mathrm{MF} \\
(\text { cols. } / 100 \mathrm{~mL})\end{array}$ & 7 & 0 & 7 & 10 & 27 & 85 & 690 & 1,200 \\
\hline $\begin{array}{l}\text { Solids, residue at } 105^{\circ} \mathrm{C}, \\
\text { suspended }(\mathrm{mg} / \mathrm{L})\end{array}$ & 9 & 1 & 8 & $<1$ & $\mathrm{e} 3.0$ & $\mathrm{e} 5.0$ & $\mathrm{e} 7.5$ & 10 \\
\hline $\begin{array}{l}\text { Nitrogen, nitrite plus } \\
\text { nitrate, dissolved (as N) }\end{array}$ & 5 & 0.05 & 5 & 0.35 & -- & 0.40 & -- & 0.55 \\
\hline $\begin{array}{l}\text { Nitrogen, ammonia } \\
\text { plus organic, total (as } \mathrm{N} \text { ) }\end{array}$ & 7 & 0.1 & 1 & -- & -- & -- & -- & 0.30 \\
\hline Phosphorus, total (as P) & 7 & 0.05 & 6 & $<0.05$ & $\mathrm{e} 0.01$ & $\mathrm{e} 0.02$ & $\mathrm{e} 0.03$ & 0.03 \\
\hline
\end{tabular}


Table 28. Statistical summary of selected water-quality data collected at station 212329157483102, Waimaluhia Reservoir cross-section 3-2 at $1 \mathrm{ft}$ depth near Kaneohe, Oahu, Hawaii, from September 1983 to January 1998

[inst., instantaneous; $\mu \mathrm{S} / \mathrm{cm}$ at $25^{\circ} \mathrm{C}$, microsiemens per centimeter at 25 degrees Celsius; $\mathrm{mg} / \mathrm{L}$, milligram per liter; $\mu \mathrm{g} / \mathrm{L}$, microgram per liter; <, less than; e, estimated using log-probability regression; $0.7 \mu \mathrm{m}-\mathrm{MF}, 0.7$ micron membrane filter; cols./100 mL, colonies per 100 milliliters; $\mathrm{ft}^{3} / \mathrm{s}$, cubic foot per second; ${ }^{\circ} \mathrm{C}$, degrees Celsius; NTU, nephelometric turbidity unit; --, no data or not computed; Range of dates are the time periods of water-quality data collection during the specified construction activity]

\begin{tabular}{|c|c|c|c|c|c|c|c|c|}
\hline Property or constituent & $\begin{array}{l}\text { Number } \\
\text { of } \\
\text { samples }\end{array}$ & $\begin{array}{l}\text { Minimum } \\
\text { detection } \\
\text { limit }\end{array}$ & $\begin{array}{l}\text { Number } \\
\text { of } \\
\text { detections }\end{array}$ & $\begin{array}{l}\text { Mini- } \\
\text { mum }\end{array}$ & $\frac{\text { Conce }}{25}$ & $\frac{\text { tration pe }}{50}$ & $\frac{\text { centile }}{75}$ & $\begin{array}{l}\text { Maxi- } \\
\text { mum }\end{array}$ \\
\hline \multicolumn{9}{|c|}{ During construction, September 1983 to June 1992, physical, biological, and nutrients } \\
\hline $\begin{array}{l}\text { Specific conductance } \\
\left(\mathrm{uS} / \mathrm{cm} \text { at } 25^{\circ} \mathrm{C}\right)\end{array}$ & 23 & 1 & 23 & 156 & 181 & 184 & 191 & 209 \\
\hline $\mathrm{pH}$ (units) & 23 & 0.1 & 23 & 7.1 & 7.4 & 7.6 & 7.8 & 7.1 \\
\hline Temperature, water $\left({ }^{\circ} \mathrm{C}\right)$ & 23 & 0.5 & 23 & 21.4 & 24.5 & 26.3 & 26.6 & 28.1 \\
\hline Turbidity (NTU) & 21 & 0.1 & 21 & 0.50 & 0.75 & 1.0 & 1.6 & 31 \\
\hline Oxygen dissolved (mg/L) & 23 & 0.1 & 23 & 6.0 & 7.2 & 7.6 & 8.0 & 9.6 \\
\hline $\begin{array}{l}\text { Fecal coliform } 0.7 \mu \mathrm{m}-\mathrm{MF} \\
\text { (cols. } / 100 \mathrm{~mL})\end{array}$ & 11 & 0 & 11 & 1 & 2 & 8 & 9 & 59 \\
\hline $\begin{array}{l}\text { Solids, residue at } 105^{\circ} \mathrm{C}, \\
\text { suspended }(\mathrm{mg} / \mathrm{L})\end{array}$ & 12 & 1 & 8 & $<1$ & e1.1 & $\mathrm{e} 3.0$ & e 3.8 & 12 \\
\hline $\begin{array}{l}\text { Nitrogen, nitrite plus } \\
\text { nitrate, total (as N) }\end{array}$ & 11 & 0.05 & 9 & $<0.1$ & $\mathrm{e} 0.20$ & $\mathrm{e} 0.30$ & $\mathrm{e} 0.35$ & 0.40 \\
\hline $\begin{array}{l}\text { Nitrogen, nitrite plus } \\
\text { nitrate, dissolved (as N) }\end{array}$ & 0 & 0.05 & -- & -- & -- & -- & -- & -- \\
\hline $\begin{array}{l}\text { Nitrogen, ammonia } \\
\text { plus organic, total (as } \mathrm{N} \text { ) }\end{array}$ & 11 & 0.2 & 9 & $<0.2$ & $\mathrm{e} 0.20$ & $\mathrm{e} 0.30$ & $\mathrm{e} 0.40$ & 0.60 \\
\hline Phosphorus, total (as P) & 11 & 0.01 & 9 & $<0.01$ & $\mathrm{e} 0.01$ & $\mathrm{e} 0.01$ & $\mathrm{e} 0.02$ & 0.03 \\
\hline \multicolumn{9}{|c|}{ After construction, August 1992 to January 1998, physical, biological, and nutrients } \\
\hline $\begin{array}{l}\text { Specific conductance } \\
\left(\mu \mathrm{S} / \mathrm{cm} \text { at } 25^{\circ} \mathrm{C}\right)\end{array}$ & 15 & 1 & 15 & 144 & 184 & 186 & 194 & 200 \\
\hline $\mathrm{pH}$ (units) & 15 & 0.1 & 15 & 7.2 & 7.5 & 7.6 & 7.7 & 7.8 \\
\hline Temperature, water $\left({ }^{\circ} \mathrm{C}\right)$ & 15 & 0.5 & 15 & 20.8 & 21.5 & 23.0 & 26.5 & 27.5 \\
\hline Turbidity (NTU) & 15 & 0.1 & 15 & 0.20 & 1.0 & 1.3 & 2.1 & 16 \\
\hline Oxygen dissolved (mg/L) & 15 & 0.1 & 15 & 6.1 & 6.9 & 7.3 & 7.7 & 8.2 \\
\hline $\begin{array}{l}\text { Fecal coliform } 0.7 \mu \mathrm{m}-\mathrm{MF} \\
(\text { cols. } / 100 \mathrm{~mL})\end{array}$ & 8 & 0 & 8 & 2 & 5 & 13 & 55 & 1,400 \\
\hline $\begin{array}{l}\text { Solids, residue at } 105^{\circ} \mathrm{C}, \\
\text { suspended (mg/L) }\end{array}$ & 9 & 1 & 8 & $<1$ & $\mathrm{e} 1.0$ & $\mathrm{e} 2.0$ & $\mathrm{e} 4.0$ & 7.0 \\
\hline $\begin{array}{l}\text { Nitrogen, nitrite plus } \\
\text { nitrate, dissolved (as N) }\end{array}$ & 5 & 0.05 & 5 & 0.30 & -- & 0.37 & -- & 0.51 \\
\hline $\begin{array}{l}\text { Nitrogen, ammonia } \\
\text { plus organic, total (as } \mathrm{N} \text { ) }\end{array}$ & 7 & 0.1 & 2 & $<0.1$ & -- & -- & -- & 0.20 \\
\hline Phosphorus, total (as P) & 7 & 0.05 & 3 & $<0.05$ & -- & -- & -- & 0.02 \\
\hline
\end{tabular}


Table 29. Statistical summary of selected water-quality data collected at station 212329157483102, Waimaluhia Reservoir cross-section 3-2 at $5 \mathrm{ft}$ depth near Kaneohe, Oahu, Hawaii, from January 1984 to January 1998

[inst., instantaneous; $\mu \mathrm{S} / \mathrm{cm}$ at $25^{\circ} \mathrm{C}$, microsiemens per centimeter at 25 degrees Celsius; $\mathrm{mg} / \mathrm{L}$, milligram per liter; $\mu \mathrm{g} / \mathrm{L}$, microgram per liter; <, less than; e, estimated using log-probability regression; $0.7 \mu \mathrm{m}-\mathrm{MF}, 0.7$ micron membrane filter; cols./100 mL, colonies per 100 milliliters; $\mathrm{ft}^{3} / \mathrm{s}$, cubic foot per second; ${ }^{\circ} \mathrm{C}$, degrees Celsius; NTU, nephelometric turbidity unit; --, no data or not computed; Range of dates are the time periods of water-quality data collection during the specified construction activity]

\begin{tabular}{|c|c|c|c|c|c|c|c|c|}
\hline Property or constituent & $\begin{array}{l}\text { Number } \\
\text { of } \\
\text { samples }\end{array}$ & $\begin{array}{l}\text { Minimum } \\
\text { detection } \\
\text { limit }\end{array}$ & $\begin{array}{l}\text { Number } \\
\text { of } \\
\text { detections }\end{array}$ & $\begin{array}{l}\text { Mini- } \\
\text { mum }\end{array}$ & \multicolumn{3}{|c|}{ Concentration percentile } & $\begin{array}{l}\text { Maxi- } \\
\text { mum }\end{array}$ \\
\hline \multicolumn{9}{|c|}{ During construction, January 1984 to June 1992, physical, biological, and nutrients } \\
\hline Specific conductance & & & & & & & & \\
\hline$\left(\mu \mathrm{S} / \mathrm{cm}\right.$ at $\left.25^{\circ} \mathrm{C}\right)$ & 20 & 1 & 20 & 157 & 180 & 184 & 193 & 209 \\
\hline $\mathrm{pH}$ (units) & 20 & 0.1 & 20 & 7.1 & 7.4 & 7.6 & 7.8 & 8.9 \\
\hline Temperature, water $\left({ }^{\circ} \mathrm{C}\right)$ & 20 & 0.5 & 20 & 21.4 & 24.2 & 26.0 & 26.6 & 27.6 \\
\hline Turbidity (NTU) & 6 & 0.1 & 6 & 0.8 & 1.0 & 1.6 & 2.5 & 3.6 \\
\hline Oxygen dissolved (mg/L) & 20 & 0.1 & 20 & 5.9 & 7.1 & 7.2 & 7.8 & 9.2 \\
\hline $\begin{array}{l}\text { Fecal coliform } 0.7 \mu \mathrm{m}-\mathrm{MF} \\
\text { (cols. } / 100 \mathrm{~mL})\end{array}$ & 2 & 0 & 2 & 16 & -- & -- & -- & 21 \\
\hline $\begin{array}{l}\text { Solids, residue at } 105^{\circ} \mathrm{C}, \\
\text { suspended }(\mathrm{mg} / \mathrm{L})\end{array}$ & 2 & 1 & 2 & 2.0 & -- & -- & -- & 4.0 \\
\hline $\begin{array}{l}\text { Nitrogen, nitrite plus } \\
\text { nitrate, total (as } \mathrm{N})\end{array}$ & 2 & 0.05 & 2 & 0.33 & -- & -- & -- & 0.35 \\
\hline $\begin{array}{l}\text { Nitrogen, nitrite plus } \\
\text { nitrate, dissolved (as N) }\end{array}$ & 0 & 0.05 & -- & -- & -- & -- & -- & -- \\
\hline $\begin{array}{l}\text { Nitrogen, ammonia } \\
\text { plus organic, total (as N) }\end{array}$ & 2 & 0.2 & 2 & 0.30 & -- & -- & -- & 0.50 \\
\hline Phosphorus, total (as P) & 2 & 0.01 & 2 & 0.02 & -- & -- & -- & 0.03 \\
\hline \multicolumn{9}{|c|}{ After construction, August 1992 to January 1998, physical, biological, and nutrients } \\
\hline $\begin{array}{l}\text { Specific conductance } \\
\left(\mu \mathrm{S} / \mathrm{cm} \text { at } 25^{\circ} \mathrm{C}\right)\end{array}$ & 15 & 1 & 15 & 146 & 184 & 188 & 194 & 200 \\
\hline $\mathrm{pH}$ (units) & 15 & 0.1 & 15 & 7.2 & 7.5 & 7.6 & 7.8 & 7.8 \\
\hline Temperature, water $\left({ }^{\circ} \mathrm{C}\right)$ & 15 & 0.5 & 15 & 20.8 & 21.5 & 22.5 & 26.5 & 27.0 \\
\hline Turbidity (NTU) & 15 & 0.1 & 15 & 0.4 & 0.8 & 1.3 & 2.2 & 15 \\
\hline Oxygen dissolved (mg/L) & 15 & 0.1 & 15 & 6.0 & 6.8 & 7.3 & 7.7 & 8.2 \\
\hline $\begin{array}{l}\text { Fecal coliform } 0.7 \mu \mathrm{m}-\mathrm{MF} \\
(\text { cols. } / 100 \mathrm{~mL})\end{array}$ & 8 & 0 & 8 & 1.0 & 3.2 & 26 & 144 & 890 \\
\hline $\begin{array}{l}\text { Solids, residue at } 105^{\circ} \mathrm{C}, \\
\text { suspended }(\mathrm{mg} / \mathrm{L})\end{array}$ & 9 & 1 & 6 & $<1$ & $\mathrm{e} 0.85$ & $\mathrm{e} 2.0$ & e5.0 & 5.0 \\
\hline $\begin{array}{l}\text { Nitrogen, nitrite plus } \\
\text { nitrate, dissolved (as N) }\end{array}$ & 5 & 0.05 & 5 & 0.30 & -- & 0.39 & -- & 0.48 \\
\hline $\begin{array}{l}\text { Nitrogen, ammonia } \\
\text { plus organic, total (as N) }\end{array}$ & 7 & 0.1 & 1 & -- & -- & -- & -- & 0.20 \\
\hline Phosphorus, total (as P) & 7 & 0.05 & 3 & $<0.05$ & -- & -- & -- & 0.02 \\
\hline
\end{tabular}


Table 30. Statistical summary of selected water-quality data collected at station 212329157483102, Waimaluhia Reservoir cross-section 3-2 at $10 \mathrm{ft}$ depth near Kaneohe, Oahu, Hawaii, from May 1983 to January 1998

[inst., instantaneous; $\mu \mathrm{S} / \mathrm{cm}$ at $25^{\circ} \mathrm{C}$, microsiemens per centimeter at 25 degrees Celsius; $\mathrm{mg} / \mathrm{L}$, milligram per liter; $\mu \mathrm{g} / \mathrm{L}$, microgram per liter; <, less than; e, estimated using log-probability regression; $0.7 \mu \mathrm{m}-\mathrm{MF}, 0.7$ micron membrane filter; cols./100 mL, colonies per 100 milliliters; $\mathrm{ft}^{3} / \mathrm{s}$, cubic foot per second; ${ }^{\circ} \mathrm{C}$, degrees Celsius; NTU, nephelometric turbidity unit; --, no data or not computed; Range of dates are the time periods of water-quality data collection during the specified construction activity]

\begin{tabular}{|c|c|c|c|c|c|c|c|c|}
\hline \multirow[b]{2}{*}{ Property or constituent } & \multirow{2}{*}{$\begin{array}{l}\text { Number } \\
\text { of } \\
\text { samples }\end{array}$} & \multirow{2}{*}{$\begin{array}{l}\text { Minimum } \\
\text { detection } \\
\text { limit } \\
\end{array}$} & \multirow{2}{*}{$\begin{array}{l}\text { Number } \\
\text { of } \\
\text { detections }\end{array}$} & \multirow{2}{*}{$\begin{array}{l}\text { Mini- } \\
\text { mum }\end{array}$} & \multicolumn{3}{|c|}{ Concentration percentile } & \multirow{2}{*}{$\begin{array}{l}\text { Maxi- } \\
\text { mum }\end{array}$} \\
\hline & & & & & 25 & 50 & 75 & \\
\hline \multicolumn{9}{|c|}{ During construction, May 1983 to June 1992, physical, biological, and nutrients } \\
\hline Specific conductance & & & & & & & & \\
\hline$\left(\mu \mathrm{S} / \mathrm{cm}\right.$ at $\left.25^{\circ} \mathrm{C}\right)$ & 23 & 1 & 23 & 157 & 178 & 186 & 192 & 208 \\
\hline $\mathrm{pH}$ (units) & 23 & 0.1 & 23 & 6.6 & 7.2 & 7.4 & 7.5 & 8.8 \\
\hline Temperature, water $\left({ }^{\circ} \mathrm{C}\right)$ & 23 & 0.5 & 23 & 21.1 & 24.0 & 25.0 & 26.1 & 26.9 \\
\hline Turbidity (NTU) & 22 & 0.1 & 22 & 0.7 & 1.5 & 2.4 & 3.2 & 9.5 \\
\hline Oxygen dissolved (mg/L) & 23 & 0.1 & 23 & 1.4 & 4.9 & 6.6 & 7.0 & 8.3 \\
\hline $\begin{array}{l}\text { Fecal coliform } 0.7 \mu \mathrm{m}-\mathrm{MF} \\
(\text { cols. } / 100 \mathrm{~mL})\end{array}$ & 11 & 0 & 10 & $<1$ & $\mathrm{e} 2$ & e10 & e18 & 66 \\
\hline $\begin{array}{l}\text { Solids, residue at } 105^{\circ} \mathrm{C}, \\
\text { suspended }(\mathrm{mg} / \mathrm{L})\end{array}$ & 12 & 1 & 11 & $<1$ & $\mathrm{e} 3.0$ & e4.5 & e9.2 & 18 \\
\hline $\begin{array}{l}\text { Nitrogen, nitrite plus } \\
\text { nitrate, dissolved (as N) }\end{array}$ & 0 & 0.05 & -- & -- & -- & -- & -- & -- \\
\hline $\begin{array}{l}\text { Nitrogen, ammonia } \\
\text { plus organic, total (as N) }\end{array}$ & 11 & 0.05 & 11 & 0.20 & 0.30 & 0.30 & 0.40 & 1.0 \\
\hline Phosphorus, total (as P) & 11 & 0.05 & 9 & $<0.05$ & $\mathrm{e} 0.01$ & $\mathrm{e} 0.01$ & $\mathrm{e} 0.03$ & 0.05 \\
\hline \multicolumn{9}{|c|}{ After construction, August 1992 to January 1998, physical, biological, and nutrients } \\
\hline Specific conductance & & & & & & & & \\
\hline$\left(\mu \mathrm{S} / \mathrm{cm}\right.$ at $\left.25^{\circ} \mathrm{C}\right)$ & 13 & 1 & 13 & 162 & 184 & 188 & 192 & 200 \\
\hline $\mathrm{pH}$ (units) & 13 & 0.1 & 13 & 7.3 & 7.5 & 7.6 & 7.6 & 7.8 \\
\hline Temperature, water $\left({ }^{\circ} \mathrm{C}\right)$ & 13 & 0.5 & 13 & 20.8 & 21.5 & 23.0 & 25.4 & 27.0 \\
\hline Turbidity (NTU) & 13 & 0.1 & 13 & 0.90 & 1.2 & 2.8 & 6.7 & 17 \\
\hline Oxygen dissolved (mg/L) & 13 & 0.1 & 13 & 3.3 & 5.5 & 6.8 & 7.4 & 8.3 \\
\hline $\begin{array}{l}\text { Fecal coliform } 0.7 \mu \mathrm{m}-\mathrm{MF} \\
\text { (cols. } / 100 \mathrm{~mL} \text { ) }\end{array}$ & 7 & 0 & 7 & $<4$ & e6 & e24 & e57 & 860 \\
\hline $\begin{array}{l}\text { Solids, residue at } 105^{\circ} \mathrm{C}, \\
\text { suspended }(\mathrm{mg} / \mathrm{L})\end{array}$ & 8 & 1 & 7 & $<1$ & e3.0 & e 4.0 & e20 & 24 \\
\hline $\begin{array}{l}\text { Nitrogen, nitrite pluse } \\
\text { nitrate, total (as N) }\end{array}$ & 0 & 0.1 & -- & -- & -- & -- & -- & -- \\
\hline $\begin{array}{l}\text { Nitrogen, nitrite plus } \\
\text { nitrate, dissolved (as N) }\end{array}$ & 4 & 0.05 & 4 & 0.30 & -- & 0.38 & -- & 0.47 \\
\hline $\begin{array}{l}\text { Nitrogen, ammonia } \\
\text { plus organic, total (as N) }\end{array}$ & 6 & 0.2 & 2 & $<0.2$ & -- & -- & -- & 0.20 \\
\hline Phosphorus, total (as P) & 6 & 0.01 & 4 & $<0.01$ & -- & -- & -- & 0.09 \\
\hline
\end{tabular}


Table 31. Pesticides, herbicides, and polychlorinated compounds analyzed for at stream-gaging and water-quality stations in the H-3 Highway study area, Oahu, Hawaii, February 1983 to August 1999 [all values in microgram per liter; USGS, U.S. Geological Survey]

\begin{tabular}{lcc}
\hline \multicolumn{1}{c}{ Constituent } & $\begin{array}{r}\text { USG parameter } \\
\text { code }\end{array}$ & $\begin{array}{c}\text { Laboratory reporting } \\
\text { limit }\end{array}$ \\
\hline Aldrin & 39330 & 0.01 \\
Chlordane & 39350 & 0.1 \\
Chlorpyrifos & 38932 & 0.01 \\
DDD & 39360 & 0.01 \\
DDE & 39365 & 0.01 \\
DDT & 39370 & 0.01 \\
DEF & 39040 & 0.01 \\
Diazinon & 39570 & 0.01 \\
Dieldrin & 39380 & 0.01 \\
Disulfoton & 39011 & 0.01 \\
Endosulfan I & 39388 & 0.01 \\
Endrin & 39390 & 0.01 \\
Ethion & 39398 & 0.01 \\
Fonofos & 82614 & 0.01 \\
Heptachlor & 39410 & 0.01 \\
Heptachlor epoxide & 39420 & 0.01 \\
Lindane & 39340 & 0.01 \\
Malathion & 39530 & 0.01 \\
Methoxychlor & 39480 & 0.01 \\
Parathion-methyl & 39600 & 0.01 \\
Mirex & 39755 & 0.01 \\
Parathion & 39540 & 0.01 \\
Perthane & 39034 & 0.1 \\
Phorate & 39023 & 0.01 \\
PCB & 39516 & 0.1 \\
PCN & 39250 & 0.1 \\
Toxaphene & 39400 & 1 \\
Trithion & 39786 & 0.01 \\
2,4-D & 39730 & 0.01 \\
2,4-DP & 82183 & 0.01 \\
2,4,5-T & 39740 & 0.01 \\
Silvex & 39760 & 0.01 \\
\hline & & \\
\hline
\end{tabular}


Table 32. Pesticides, herbicides and polychlorinated compounds detected at stream-gaging and water-quality stations in the H-3 Highway study area, Oahu, Hawaii, February 1983 to August 1999

$[\mu \mathrm{g} / \mathrm{L}$, microgram per liter; $\mathrm{b}$, before construction period; d, during construction period; a, after construction period; station numbers are abbreviated, complete numbers are preceded by 16

\begin{tabular}{|c|c|c|c|c|c|c|}
\hline $\begin{array}{l}\text { Property or } \\
\text { constituent }\end{array}$ & $\begin{array}{l}\text { Station } \\
\text { number }\end{array}$ & Station name & $\begin{array}{c}\text { Total } \\
\text { number } \\
\text { of } \\
\text { samples }\end{array}$ & $\begin{array}{c}\text { Total } \\
\text { number of } \\
\text { detections }\end{array}$ & $\begin{array}{c}\text { Highest } \\
\text { concen- } \\
\text { tration } \\
\text { detected } \\
(\mu \mathrm{g} / \mathrm{L})\end{array}$ & $\begin{array}{c}\text { Time period } \\
\text { when highest } \\
\text { concentration } \\
\text { detected }\end{array}$ \\
\hline Aldrin & 272200 & Kamooalii Str below Luluku Str & 33 & 2 & 0.1 & $\mathrm{~d}$ \\
\hline \multirow[t]{3}{*}{ Chlordane } & 227100 & Halawa Str below H-1 & 16 & 1 & 0.1 & $\mathrm{~d}$ \\
\hline & 273950 & South Fork Kapunahala Str & 21 & 1 & 0.1 & $\mathrm{~d}$ \\
\hline & 274100 & Kaneohe Str & 14 & 2 & 0.1 & $\mathrm{~d}$ \\
\hline \multirow[t]{5}{*}{ Chlorpyrifos } & 227100 & Halawa Str below H-1 & 13 & 2 & 0.02 & $\mathrm{~d}$ \\
\hline & 265600 & Right Branch Kamooalii Str & 15 & 1 & 0.9 & $\mathrm{~d}$ \\
\hline & 265700 & Kamooalii Str at alt $220 \mathrm{ft}$ & 1 & 1 & 0.01 & $\mathrm{~b}$ \\
\hline & 273950 & South Fork Kapunahala Str & 15 & 1 & 0.02 & $\mathrm{~d}$ \\
\hline & 274100 & Kaneohe Str & 11 & 1 & 0.01 & $\mathrm{~d}$ \\
\hline \multirow[t]{2}{*}{ DDT } & 227100 & Halawa Str below H-1 & 16 & 1 & 0.01 & d \\
\hline & 275000 & Haiku Str & 32 & 1 & 0.08 & d \\
\hline \multirow[t]{9}{*}{ Diazinon } & 226200 & North Halawa Str nr Honolulu & 23 & 1 & 0.01 & $\mathrm{~b}$ \\
\hline & 227100 & Halawa Str below H-1 & 16 & 9 & 0.04 & d \\
\hline & 265600 & Right Branch Kamooalii Str & 26 & 1 & 0.01 & d \\
\hline & 265700 & Kamooalii Str at alt $220 \mathrm{ft}$ & 3 & 1 & 0.02 & $\mathrm{~b}$ \\
\hline & 267500 & Hooleinaiwa Str abv confluence & 6 & 1 & 0.01 & $\mathrm{~b}$ \\
\hline & 270900 & Luluku Str & 25 & 1 & 0.01 & $\mathrm{~b}$ \\
\hline & 272200 & Kamooalii Str below Luluku Str & 15 & 3 & 0.01 & $\mathrm{~d}$ \\
\hline & 274100 & Kaneohe Str & 14 & 3 & 0.01 & $\mathrm{~d}$ \\
\hline & 275000 & Haiku Str & 24 & 1 & 0.01 & $\mathrm{~d}$ \\
\hline \multirow[t]{4}{*}{ Dieldrin } & 227100 & Halawa Str below H-1 & 16 & 1 & 0.01 & $\mathrm{~d}$ \\
\hline & 272200 & Kamooalii Str below Luluku Str & 33 & 5 & 0.01 & $\mathrm{~d}, \mathrm{a}$ \\
\hline & 273950 & South Fork Kapunahala Str & 21 & 4 & 0.02 & $\mathrm{~d}$ \\
\hline & 274100 & Kaneohe Str & 14 & 13 & 0.05 & d \\
\hline Heptachlor epoxide & 274100 & Kaneohe Str & 14 & 5 & 0.01 & $\mathrm{~d}$ \\
\hline \multirow[t]{5}{*}{ Malathion } & 226200 & North Halawa Str nr Honolulu & 23 & 1 & 0.02 & $\mathrm{~d}$ \\
\hline & 227100 & Halawa Str below H-1 & 16 & 1 & 0.01 & $\mathrm{~d}$ \\
\hline & 265600 & Right Branch Kamooalii Str & 26 & 1 & 0.01 & a \\
\hline & 265700 & Kamooalii Str at alt $220 \mathrm{ft}$ & 3 & 1 & 0.28 & $\mathrm{~b}$ \\
\hline & 273950 & South Fork Kapunahala Str & 20 & 1 & 0.05 & $\mathrm{~b}$ \\
\hline \multirow[t]{5}{*}{$2,4-\mathrm{D}$} & 265600 & Right Branch Kamooalii Str & 25 & 2 & 0.02 & $\mathrm{~d}$ \\
\hline & 267500 & Hooleinaiwa Str abv confluence & 5 & 1 & 0.02 & $\mathrm{~b}$ \\
\hline & 272200 & Kamooalii Str below Luluku Str & 31 & 6 & 0.03 & d \\
\hline & 274100 & Kaneohe Str & 14 & 1 & 0.02 & $\mathrm{~d}$ \\
\hline & 275000 & Haiku Str & 23 & 1 & 0.02 & $\mathrm{~d}$ \\
\hline \multirow[t]{4}{*}{ 2,4-DP } & 227100 & Halawa Str below H-1 & 16 & 2 & 0.03 & $\mathrm{~d}$ \\
\hline & 265600 & Right Branch Kamooalii Str & 25 & 1 & 0.02 & d \\
\hline & 272200 & Kamooalii Str below Luluku Str & 31 & 3 & 0.04 & $\mathrm{~d}$ \\
\hline & 273950 & South Fork Kapunahala Str & 20 & 1 & 0.19 & $\mathrm{~d}$ \\
\hline Silvex & 226200 & North Halawa Str nr Honolulu & 23 & 1 & 0.01 & $\mathrm{~b}$ \\
\hline
\end{tabular}

